\title{
The Chemical Ecology of Primate Seed Dispersal
}

\author{
Dissertation for the award of the degree \\ Doctor of Philosophy (Ph.D.) Division of Mathematics and Natural Sciences \\ of the Georg-August-Universität Göttingen \\ within the Basic Biology Program \\ of the Georg-August University School of Science (GAUSS)
}

Submitted by Omer Nevo

Place of birth: Jerusalem

Göttingen 2015 


\section{THESIS COMMITTEE}

Eckhard W. Heymann, Behavioral Ecology \& Sociobiology Unit, German Primate Center

Peter Kappeler, Behavioral Ecology \& Sociobiology Unit, German Primate Center

\section{MEMBERS OF THE EXAMINATION BOARD}

Reviewer: Eckhard W. Heymann, Behavioral Ecology \& Sociobiology Unit, German Primate Center

Second reviewer: Peter Kappeler, Behavioral Ecology \& Sociobiology Unit, German Primate Center

\section{FURTHER MEMBERS OF THE EXAMINATION BOARD}

Manfred Ayasse, Institute of Evolutionary Ecology and Conservation Genomics, University of Ulm

Oliver Schülke, CRC Evolution of Social Behavior, University of Göttingen

Claudia Fichtel, Behavioral Ecology \& Sociobiology Unit, German Primate Center

Dietmar Zinner, Cognitive Ethology Unit, German Primate Center

Christian Ross, Primate Genetics Laboratory, German Primate Center

Date of the oral exam: 8 May 2015 


\section{SUMMARY}

Many tropical angiosperms rely on frugivores for seed dispersal and evolved fleshy fruits to attract them. Although both sides of the interaction are generalists, frugivores do not feed on the full range of fruit species in their habitats and angiosperms rely on the dispersal services of only a share of the frugivore community. This observation led to the "Dispersal Syndrome Hypothesis", which postulates that over time fruits evolve to specialize on certain guilds of frugivorous seed dispersers and consequently their traits evolve in response to the dietary and sensory capacities of their main dispersal agents.

Fruit traits such as size, seed size and husk thickness have been shown to be malleable to selection pressures exerted by their main seed-dispersal vectors. Additionally, due to competition for dispersal services and the need to promote consumption of ripe fruits and thus dispersal of mature seeds, fruits are also under selection pressures to provide reliable signals for ripeness. A prime example is fruit color, which has evolved independently in many bird-dispersed species to signal ripeness and possibly nutrient content. Fruit odor, similarly, has been speculated to be a signaling system between plants and frugivores with elaborated olfactory capabilities, but this has only recently received support in figs (genus Ficus) dispersed by bats. Yet data are still restricted to the narrow bat-fig model system and it is not clear whether fruit olfactory signaling has evolved in other plant genera and in the communication with other taxa, and thus indeed a recurring component of some Dispersal Syndromes.

Primates are one of the most important seed dispersal vectors in the tropics. Until recently, their olfactory capabilities were considered low and thus irrelevant for the study of their feeding ecology. This view has been utterly revisited over the past years and primates are now known to possess high olfactory capacities. Therefore, under the framework of the Dispersal Syndrome Hypothesis, it is likely that fruits whose seeds they disperse evolved olfactory signals for ripeness, too.

This thesis explores the evolution and functions of fruit aroma in the communication between primates and plants. The first chapter is a theoretical review regarding the roles of olfaction in primate feeding ecology. It concludes, based on available behavioral works, that frugivory is the 
dietary category that requires most reliance on olfaction in feeding primates, and that olfactory cues are used solely for the function of fruit selection from very short distances. So, primatedispersed fruits are expected to be under pressure to provide signals that are not necessarily carried away to long distances, but rather maximize the ability to identify ripe fruits from close proximity.

The second chapter is a comparative analysis of fruit odor in four Neotropical plant species, two dispersed by primates and two by birds. It demonstrates that ripe primate-dispersed fruits bear odors that are strong, compound-rich and significantly different from the odors of unripe fruits. Thus, their odors provide a reliable signal for ripeness that could be used for fruit selection. In contrast, bird-dispersed fruits emit relatively weak and compound-poor odors that are not different from the odors of unripe fruits. Hence, their odor profiles are not informative regarding their level of ripeness. Since each bird-dispersed species is phylogenetically closer to one primate-dispersed species than they are to one another, these patterns of odor release are independent of phylogeny. Therefore, the chapter concludes that fruit odor as a reliable signal for ripeness evolved in these two primate-dispersed species, independent of phylogeny and hence it is likely to constitute an adapted communication system with seed-dispersing primates.

The third chapter reports olfactory-discrimination experiments that confirmed that primates indeed "understand" the signal - that they can physiologically discriminate between odors of ripe and unripe primate-dispersed fruits and choose ripe fruits based on their odor in the absence of cues from other trajectories. It further reports experiments which show that no single compound or compound class in the odor of fruits is significantly more important than others to allow ripe-fruit recognition.

The final section provides a synthesis of the results and a framework for integrating the study of primate olfaction and fruit chemistry into a new "chemical ecology of primate-plant interactions". 


\section{ACKNLOWLEDGMENTS}

First and foremost, I would like to thank the main supervisor of the project, Prof. Eckhard Heymann, for taking me as a PhD student and giving me the support, help, advice and ideas in all phases of the project - from funding acquisition, through data collection to the final writeup. I would also like to thank my second supervisor, Prof. Peter Kappeler, for the support both as a supervisor and head of the department, and mainly for running a scientifically capable and friendly department that has been a perfect environment for conducting my first major research project. Special thanks to Prof. Manfred Ayasse, who was not a supervisor de jure, but participated in all phases of the project, taught me all the methods in chemical ecology and hosted me in his lab numerous times. Whatever I achieve in the future as a scientist - I owe it to you three.

I would further like to thank the numerous collaborators in the project: Prof. Stefan Schulz, who helped with the chemical analyses; Prof. Matthias Laska, Dr. Laura Teresa Hernandez Salazar and Rosa Orts Garri - who all helped in devising and conducted the bioassays reported in the last chapter.

Thanks to all who helped during the field work: Ney Shahuano Tello and Santiago Cariajano (did I forget Camillo? Oops), and to those who shared time in the field and in lquitos - namely Darja. Also in Peru -special thanks to the Peruvian Ministry of Production and Grupo Scharf, whose utter incompetence taught me a valuable lesson.

Further thanks to the two funding agencies who paid for (most) of the work: the Minerva Foundation, who paid my salary and the DFG, who paid for other project expenses.

To all members of the Behavioral Ecology \& Sociobiology Unit of the German Primate Center - it has been a pleasure working alongside you for the past 4 years. Thanks for the friendly atmosphere and for the many interesting discussions. Similarly, to the members of the Institute for Evolutionary Ecology and Conservation Genetics, University of Ulm (née Experimental Ecology) - thanks for being such good hosts and for helping me to learn all lab methods. I look forward for working with you. 
On a more personal note, I would like to thank my wife, Nele, for all the love and support, and mainly for accepting the fact that I had to spend 11 months in the jungle. Without your support, I could not even imagine continuing with this kind of work and going to the jungle again (you brought this on yourself!). To my unborn son - thanks for stressing me to finish writing fast so I can move in time to make a room for you, and for being so understanding that Papa doesn't have a real job (no, you can't have that toy. Ask your grandfather). Now show some respect! And it's "DR FATHER", by the way.

Finally, many thanks to my dear family for being behind me from the beginning of my studies up until this very timely ending of an era too long with a student card in my wallet.

And to The Cat. 


\section{TABLE OF CONTENTS}

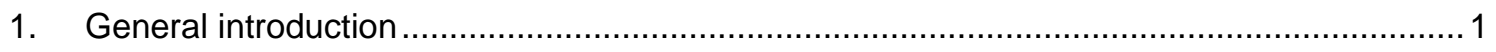

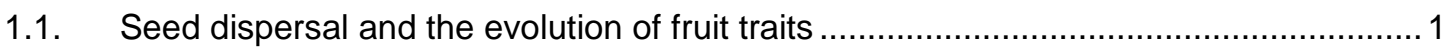

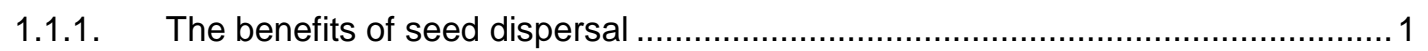

1.1.2. The evolution of fruit traits in the context of plant-frugivore interactions.................. 3

1.1.3. Fruit odor - an evolved signal for fruit ripeness? .............................................. 5

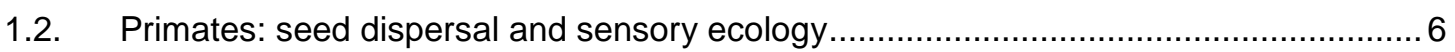

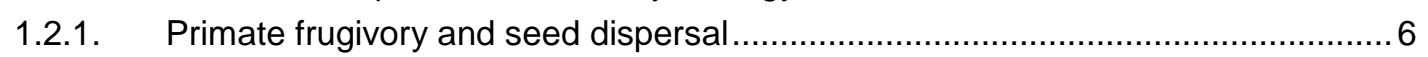

1.2.2. The effects of primate feeding ecology on fruit characteristics ............................ 9

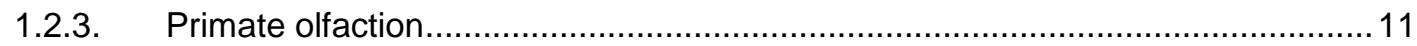

1.3. Synthesis: the chemical ecology of primate seed dispersal .................................... 13

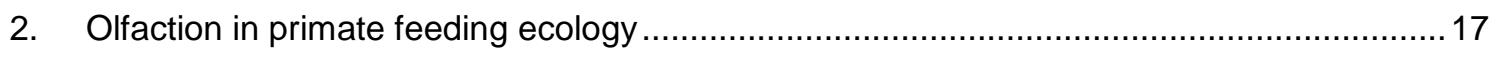

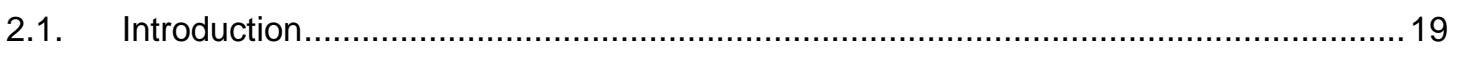

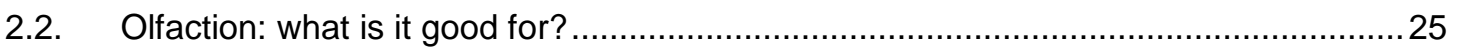

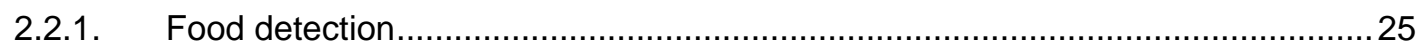

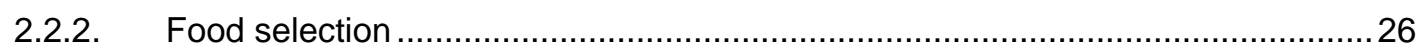

2.2.3. Summary: the function of olfaction in food acquisition ......................................28

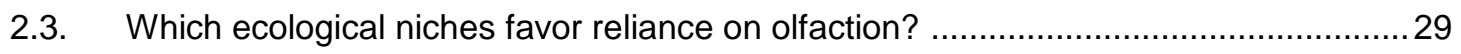

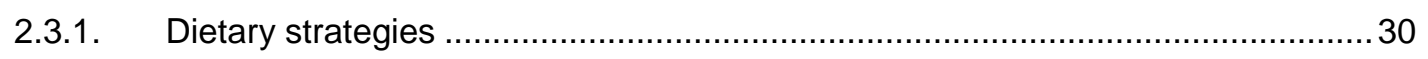

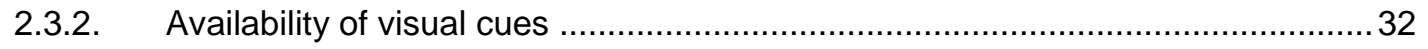

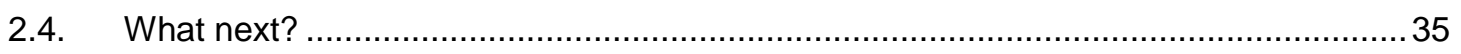

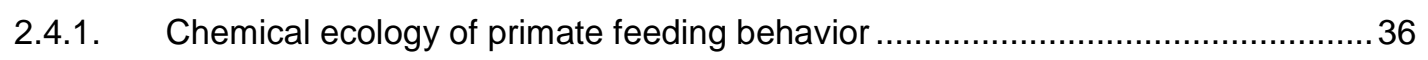

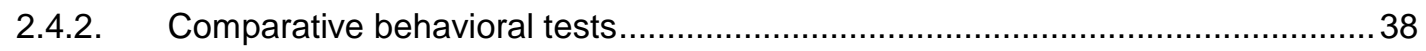

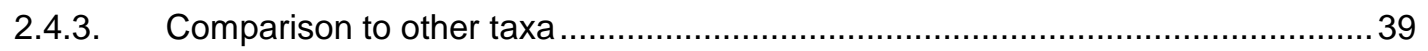

3. Fruit aroma: adaptation to primate seed dispersal? ..................................................... 41

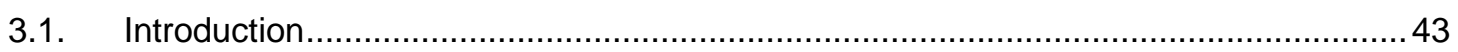

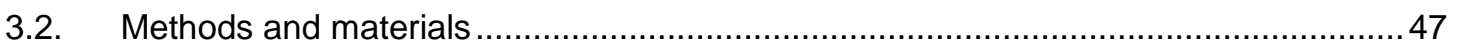

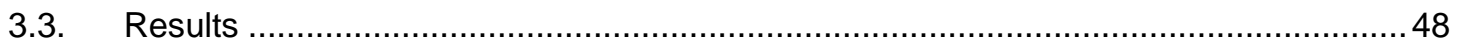

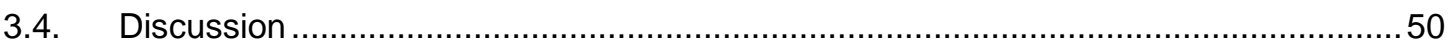

3.4.1. How to continue? A roadmap for inferring whether fruit odor is an evolved signal

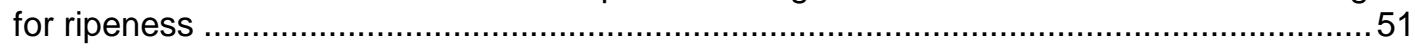

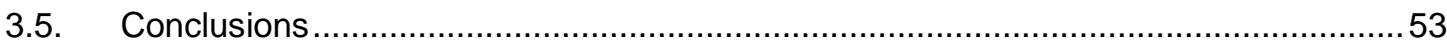

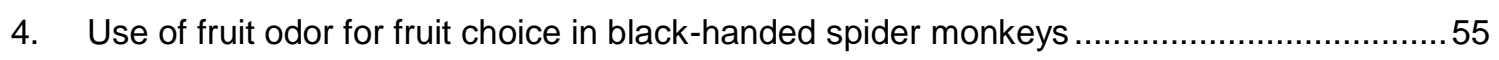

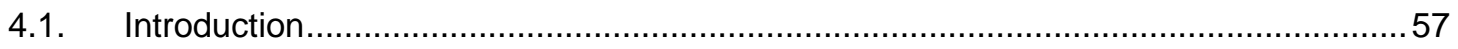

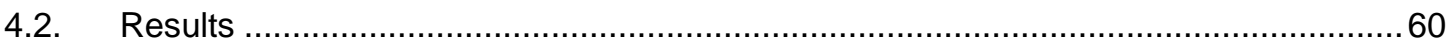

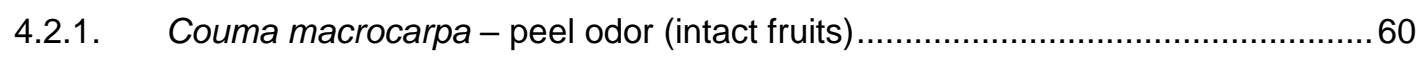

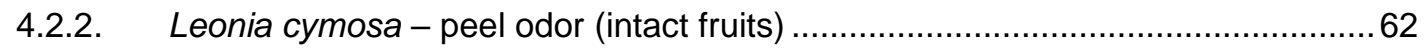

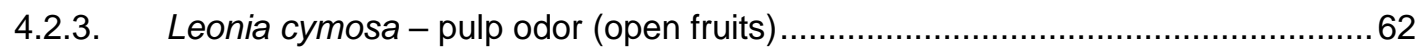

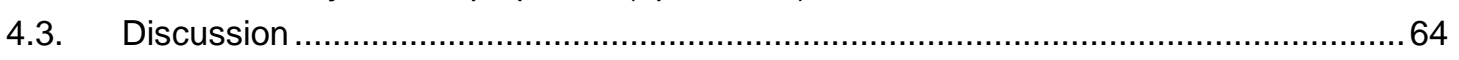

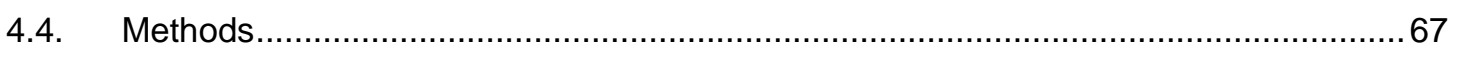

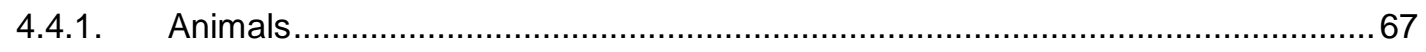

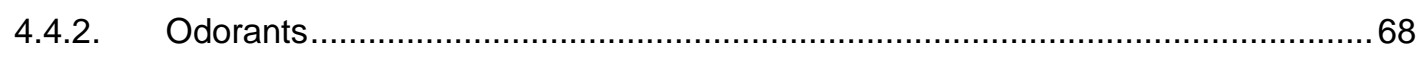

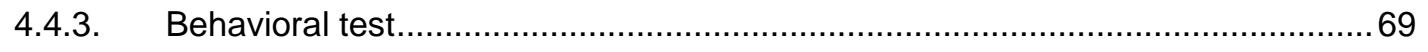




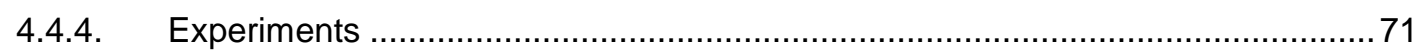

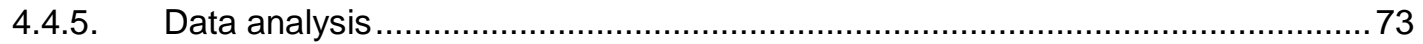

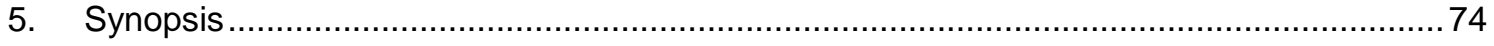

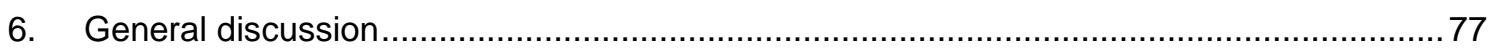

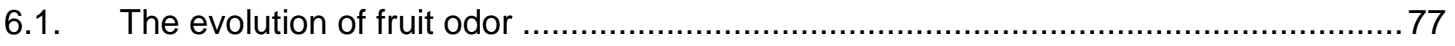

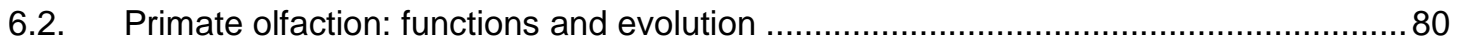

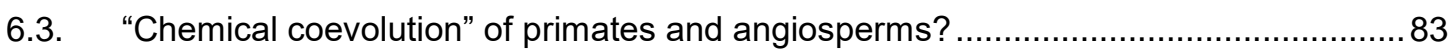

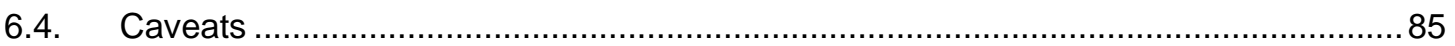

6.5. Concluding remarks: towards an evolutionary chemical ecology of primate plant

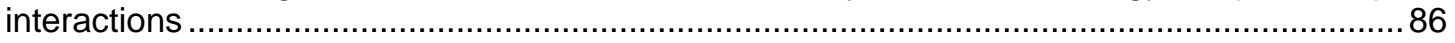

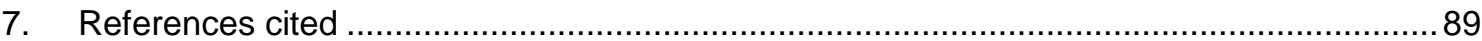

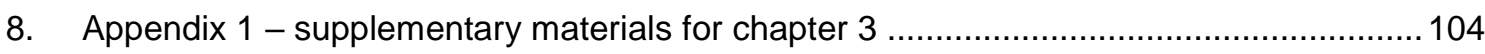

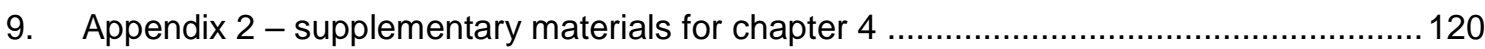

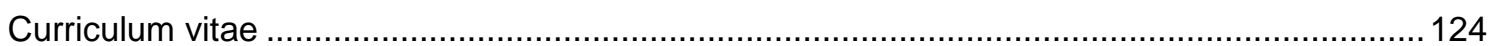

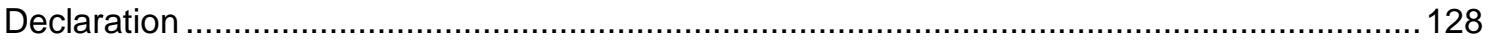




\section{GENERAL INTRODUCTION}

\subsection{Seed dispersal and the evolution of fruit traits}

\subsubsection{The benefits of seed dispersal}

The ability to disperse seeds away from the mother tree promotes an individual plant's fitness in many ways. Seeds suffer disproportionally high mortality rates when deposited in dense clusters around the mother tree, mainly due to an overly exposure to species-specific pathogens or predators and to competition with kin (Janzen 1970; Connell 1971). Dispersal away from the mother tree allows seeds to colonize open habitats and, potentially but rarely, to reach preferred microsites through "directed dispersal" (Howe and Smallwood 1982; Wenny 2001; Howe and Miriti 2004). Further, seed dispersal promotes gene flow that counters the formation of spatial-genetic structure (i.e. the tendency of spatially close plants to be genetically similar) and thus decreases the probability of inbreeding and homozygosity. Its contribution to the spatial reshuffling of alleles within a population -the reduction in spatial-genetic structure - is roughly double than that of pollination because seeds are diploid and pollen is haploid (Hamrick and Trapnell 2011). This process yields the template for recruitment of future generations (Nathan and Muller-Landau 2000) and is therefore a key factor in maintaining of ecological systems.

Contemporary and extinct plants have employed different strategies to achieve seed dispersal (van der Pijl 1982). The most primitive dispersal modes were abiotic - namely by wind or water (Tiffney 1986). Biotic dispersal probably originated in the Permian (ca. 299 - 252 my bp) or even slightly earlier, although in early stages diaspores were probably swallowed accidentally by herbivores (Tiffney 2004). Fleshy fruits - i.e. fruits that are covered with a soft, nutritious tissue that attracts frugivores - first appeared during the Mesozoic, even though earliest fleshy tissue has not necessarily served as a reward for dispersal agents (Mack 2000). The rewarding role of fruit flesh - its function as an attractant to seed-dispersal agents - may have evolved secondarily out of the non-rewarding flesh and became the predominant seed dispersal strategy in angiosperms after their major radiations of the Tertiary, which were accompanied with radiations 
of frugivorous dispersal agents such as passerine birds, bats and primates (Sussman 1991; Tiffney 2004; Sussman et al. 2013). Fleshy fruits have evolved many times independently in most angiosperm families deep into the Tertiary (Bremer and Eriksson 1992; Bolmgren and Eriksson 2005; Eriksson 2014). In contemporary ecological systems biotic seed dispersal is present, although not necessarily exclusively, in over a half of angiosperm families (Tiffney and Mazer 1995) and the vast majority of tropical taxa produce fleshy fruits and rely on endozoochory for seed dispersal (Howe and Westley 1988).

This heavy focus on endozoochory is not surprising given its many advantages to efficient dispersal. The main factor that has promoted an independent evolution of fleshy fruits is that abiotic dispersal faces an inevitable tradeoff between dispersal distance and seed quality. On one hand, the greater the distance seeds are dispersed away from the mother tree, the less likely they are to be deposited next to conspecifics and kin and hence suffer from density-dependent mortality (Janzen 1970; Connell 1971). On the other hand, this distance is negatively correlated with seed mass because heavier seeds are more difficult to disperse through abiotic means. Small seeds contain less energy and are therefore less likely to establish in shaded environments where photosynthetic efficiency is lower. As a result, larger seeds are less likely to escape densitydependent mortality, but are more likely to establish in low-light conditions. Biotic seed dispersal breaks away from this tradeoff by allowing the dispersal of large, energy-rich, seeds to greater distances (Tiffney 1984, 2004; Leishman et al. 2000; Bolmgren and Eriksson 2005, 2010). Thus, not surprisingly, the independent evolution of fleshy fruits in many taxa is associated with environmental changes to more shaded conditions (Bolmgren and Eriksson 2005) and in contemporary dense tropical forests endozoochory is by far the most important mean of seed dispersal (Howe and Westley 1988). Once established, dispersal by biotic factors can provide several other benefits to the plant. First, seed passage in the gut of the frugivore can increase the probability of germination (Traveset and Verdú 2002). Second, the deposition of seeds along with fecal material often leads to their secondary dispersal by dung beetles, which bury the seeds and so protect them from seed predators (Culot et al. 2010a). 


\subsubsection{The evolution of fruit traits in the context of plant-frugivore interactions}

Given these benefits, it is not surprising that the production of energetically costly fleshy fruits was selected in so many angiosperms, especially in dense tropical forests. Accordingly, the notion that

rewarding fleshy fruits, as a whole, have adapted in response to selection pressures exerted by frugivores is not controversial. However, whether or not particular characteristics of fruits can be attributed to their interactions with frugivores is still a matter of debate. The frugivore community is often very diverse and consequently seed treatment by the different frugivores is very different. For example, a highly durable seed's coat can protect it from rough treatment in the gut, but also inhibit germination if not reduced by the same gut treatment (McKey 1975). Thus, all fleshy-fruit producing plants face a tradeoff. On one hand, they could produce generalist fruits that are consumed by many different frugivores but provide poor dispersal services due to, for example, a mismatch between the seed coat and the gut treatment by many consumers. Alternatively, they could produce specialized fruits that are accessible to only a share of the frugivore community, and so suffer from a smaller number of dispersal vectors but achieve higher quality through a better match between seed and frugivore traits (McKey 1975).

A major point of disagreement is where on this specialist-generalist spectrum most fruits lie. Frugivore-dispersed fruits come in a great variety of sizes, shapes and colors (van Roosmalen 1985a; Howe and Miriti 2004). Rather than being randomly distributed across taxa, these traits tend to be correlated with one another and go hand in hand with dispersal by only a share of the frugivore community (Janson 1983; Gautier-Hion et al. 1985; Voigt et al. 2004; Lomáscolo et al. 2010). Frugivores, in turn, tend to be selective regarding the fruits they consume (Julliot 1996a; Flörchinger et al. 2010) and the major frugivore guilds vary in their sensory capacities, physiological requirements and food acquisition strategies. Given the differences between frugivores, any fruit trait faces a tradeoff because being attractive to one dispersal vector would render the fruit less attractive to another. For example, to be attractive to small birds with little handling capacities, fruits ought to be small enough to fit their gape width (Wheelwright 1993; Galetti et al. 2013). Such small fruits may be relatively unattractive to large-bodied frugivores. 
This reality led to the "Dispersal Syndrome Hypothesis" (van der Pijl 1982; Janson 1983), which postulates that even in a system of generalists, plants would be forced to specialize on dispersal by only a share of the frugivores community. Thus, the main prediction of the Dispersal Syndrome Hypothesis is that fruit traits would be under selection pressures exerted by the sensory capacities and physiological requirements of their main dispersal vectors.

The main evidence for the validity of the Dispersal Syndrome Hypothesis derives from the correlations between fruit characteristics and fruit choice by frugivores (Janson 1983; GautierHion et al. 1985; Lord 2002; Poulsen et al. 2002; Link and Stevenson 2004). These adaptationist views have, however, been criticized. First, some of these correlations disappear once phylogeny is controlled for, which means that much of the variation in fruit characteristics is more easily explained by common ancestry than by adaptation (Jordano 1995). Second, abiotic factors can strongly affect fruit traits (Bollen et al. 2005). Finally, fruit characteristics that were ascribed to selection pressures by some frugivores can actually be found in regions that they do not inhabit which puts a question mark over the causal link between frugivore behavior and fruit traits evolution (Fischer and Chapman 1993). These issues were at least partly addressed in later studies that still found support for frugivore-driven adaptations in fruit traits (Lomáscolo et al. 2008, 2010; Flörchinger et al. 2010; Lomáscolo and Schaefer 2010; Donatti et al. 2011; Valido et al. 2011). Thus, the Dispersal Syndrome Hypothesis - i.e. the notion that fruit traits are shaped by selection pressures exerted by frugivorous seed-dispersers - still offers a strong explanatory and predictive model for the evolution of fruit traits (Schaefer and Ruxton 2011). Nonetheless, the criticisms serve as a reminder that adaptation cannot be inferred as a default explanation and that many confounding factors should to be considered.

The evidence for and against the Dispersal Syndrome Hypothesis were almost fully based on comparative analysis of physical and morphological characteristics of fruits such as their size, shape and husk thickness (Janson 1983; Gautier-Hion et al. 1985; Fischer and Chapman 1993; Jordano 1995; Lord 2002). Another fruit trait that has been shown to correlate with dispersal vector and to have probably evolved in this context is fruit color (Lomáscolo and Schaefer 2010). 
Conspicuous fruit colors are common in fruits dispersed by birds (Janson 1983; Herrera 2002; Link and Stevenson 2004), who are tetrachromatic and probably possess the most acute colordiscrimination capacity among vertebrates (Bennett and Théry 2007). Fruit color has also been shown to promote consumption by birds and thus mediate the mutually beneficial interaction of birds and plants (Geravis et al. 1999; Cazetta et al. 2007). Finally, fruit color is at least partially correlated with nutrient content, thus making it a reliable signal for reward quality (Cazetta et al. 2011; Schaefer et al. 2014). So, fruit color is assumed to have evolved as a reliable signal for fruit ripeness: by allowing birds to easily identify ripe fruits and possibly their quality, it promotes ingestion of the ripe fruits seed dissemination. Reliable communication between birds and plants translates into fitness benefits for both parties.

\subsubsection{Fruit odor - an evolved signal for fruit ripeness?}

Similarly to color, fruit odor has a potential to signal ripeness and quality to seed-dispersal vectors if they are capable of detecting the olfactory signal and using it for food acquisition. Given the similarity between color and odor, this hypothesis is almost a trivial extension of the Dispersal Syndrome Hypothesis. In fact, the notion that fruit odor is a part of the signaling syndrome of fruit dispersed by olfactory guided frugivores has often been presented as if it had been fully established (Howe and Westley 1986, 1988).

However, analysis of wild fruits odor - the prerequisite for testing this hypothesis - has been extremely rare and the vast majority of studies focused on cultivated species (Rodríguez et al. 2013), whose odor profiles have been under artificial selection for generations and are therefore uninformative with regards to the ecological function of fruit odor. Further, the very idea that fruit secondary metabolites, volatile and non-volatile, fulfill any function is not fully established. The reason why they are labeled as "secondary" is that when first identified, they were considered to have no clear function in plant physiology (Schaefer and Ruxton 2011). While it is now clear that secondary compounds have many, mainly defensive, functions in leaves (Farmer 2014), their 
presence in fruits has been considered by some to be a result of pleiotropic constraints (i.e. their synthesis in leaves) and hence non-adaptive (Eriksson and Ehrlén 1998).

Thus, despite many speculations regarding potential adaptive functions of fruit secondary metabolites (Cipollini and Levey 1997), the hypothesis that fruit odor - i.e. its volatile secondary metabolites profile - is an adapted signal to frugivores has remained practically untested until quite recently (Herrera 2002) and was finally addressed as it was shown that fruit odor in batdispersed figs (genus Ficus) tends to be stronger and more unique (i.e. different from the odor of unripe fruits) than in bird-dispersed species and that bats direct their foraging efforts according to this olfactory signal (Hodgkison et al. 2007, 2013; Borges et al. 2008, 2011, 2013; Lomáscolo et al. 2010). So, it is now established that fruit odor promotes fig consumption and seed dispersal by bats and that it is likely to have adapted in response to selection pressures by frugivorous bats similarly to fruit color in bird-dispersed species.

Yet despite of the support this hypothesis received, it constitutes a beginning rather than an end of the investigation of the role chemical communication takes in the interaction between fruits and seed dispersers. All studies focused on the narrow bat-fig model system and, therefore, there is so far no evidence that olfactory signals for ripeness have evolved in other plant families and as a result of interaction with other frugivore guilds - thus indeed a recurring component in many Dispersal Syndromes.

\subsection{Primates: seed dispersal and sensory ecology}

\subsection{1. $\quad$ Primate frugivory and seed dispersal}

Primates and plants are involved in a myriad of different interactions. These range between antagonistic interactions such as herbivory (Dittus 1985) or granivory (seed predation) (Palminteri et al. 2012) and mutualistic interactions such as seed dispersal (Chapman and Onderdonk 1998) and occasionally pollination (Heymann 2011). Of the latter two, pollination is rather restricted and 
the more common primate-flower interaction is florivory (flower predation) (Heymann 2011). Seed dispersal, on the other hand, is probably the most prominent interaction between primates and plants. Fruits are by far the most important feeding item in the primate order as a whole: roughly 3 out of 4 primate species eat fruits at least occasionally, and in $40 \%$ of the species fruits contribute to at least $50 \%$ of the diet (Hohmann 2009). Thus, even species that are often categorized as mainly folivorous or insectivorous often include ripe fruits in their diets and provide seed dispersal services to plants.

This strong reliance on fruits, along with the fact that primates constitute a significant share (up to 40\%) of the frugivore biomass in the tropics (Chapman 1995; Chapman and Russo 2007), implies that primates have, one way or the other, a significant effect on the reproductive success of plants. Even when only ripe fruits are consumed (i.e. when the seeds are fully viable), several aspects affect the quality of the seed dispersal services - and, compared to alternative dispersal agents, determine whether the interaction leads to effective seed dispersal or to seed waste (Schupp 1993; Schupp et al. 2010). Seed-dispersal effectiveness (henceforth SDE) is defined as the product of two main factors: the quantity (how many seeds are dispersed?) and quality of dispersal (the probability that a single dispersal event would lead to the establishment of an adult plant). The quantity variable is determined by the number of visits to a given plant species and the number of seeds removed per visit, which are in turn a function of several variables: body size, handling behavior, local abundance and the degree of frugivory. The quality fraction is less straightforward to estimate, and relies on the treatment the seed receives in the mouth and gut, and on its deposition: the deposition location and microenvironment, whether it is deposited with many other con- and heterospecific seeds and whether it is surrounded by fecal material, which may promote secondary dispersal (Schupp et al. 2010).

So what is the seed dispersal effectiveness of primates? The overall effectiveness is not trivial to quantify (Chapman et al. 2013) and is anyway relative because in the absence of alternatives, even a low-SDE dispersal vector would be better than nothing. Regarding the quantity component of SDE, primates, and especially large-bodied species, were reported to disperse excessive 
amounts of seeds that amount to 25,000 seeds $/ \mathrm{km}^{2} /$ day by woolly monkeys (Stevenson 2000 ) or 16,000 seeds $/ \mathrm{km}^{2} /$ day by a single Bornean gibbons group (McConkey et al. 2002). These figures are often translated into a higher proportion of seeds dispersed relative to other dispersal vectors such as birds (Howe 1980; Clark et al. 2005), thus implying that at least for the quantity component of SDE, primates can be considered "good dispersal vectors". On the other hand, primates also employ a rather wasteful feeding strategy that often leads to substantial seed waste (Howe 1980).

The quality component of SDE is even more difficult to quantify. The effect of primate gut-passage treatment on germination probability is, on average, positive but lower than that of birds and bats (Traveset 1998; Traveset and Verdú 2002). However, the removal of pulp, even without swallowing the seeds, can significantly improve the survival and germination rates in some species (Lambert 2001). Further, primates, with body masses that apart from few outliers range between few hundreds of grams and several kilograms (Smith and Jungers 1997), are larger than other seed dispersers such as passerines (Martin et al. 2011) or bats (Thomas 1984) and therefore tend to move seeds to greater distances (Clark et al. 2005; Nathan et al. 2008). Additionally, larger-bodied dispersal vectors can disperse larger seeds (Kaplin and Lambert 2002) - which is one of the main advantages of endozoochory (see 1.1.1). On the other hand, the large body mass of primates also leads to the ingestion of many seeds in a single feeding bout and hence to deposition of these seeds together - a process that to some extent counters the positive effect of dispersal as means of escaping density-dependent seed mortality (Howe 1986). Finally, the deposition of seeds along with fecal material promotes secondary dispersal by dung beetles and therefore increases the probability of establishment (Vander Wall and Longland 2004,Culot et al. 2010a). Thus, although making generalizations regarding the quality component of primate SDE is impossible outside of a clear context with quantifiable reference points (e.g. alternative dispersal by birds), primates certainly have the potential to score high not only on the quantity component of SDE, but on the quality component too.

Yet another point that makes primates, as a group, a good dispersal vector is that while they show substantial variation in traits directly related to their SDE such as group size, body mass, feeding 
behavior, movement patterns and many other aspects (Campbell et al. 2010), they also show a significant overlap with regards to the fruit species they consume (Stevenson et al. 2000; Yamagiwa and Basabose 2009). Thus, plants that rely on primates for seed dispersal are usually consumed by several primate species (e.g. Janson 1983; Link and Stevenson 2004). As a result, the dispersal services that primates, as a group, provide are heterogeneous and potentially complementary. For example, specialization on dispersal by primates may provide a tree with removal of large quantities of seeds and their dispersal to great distances by larger-bodied primates, but also dispersal of a smaller number of seeds in smaller clumps. Additionally, dispersal by small primates is more likely to reach forest patches in early regeneration stages (Oliveira and Ferrari 2000,Culot et al. 2010b). All in all, through relatively high SDE quantity, positive SDE quality and diverse complementary dispersal services, primates prove to be essential for forest regeneration in many systems (Kaplin and Lambert 2002; Nuñez-Iturri and Howe 2007; NuñezIturri et al. 2008; Anzures-Dadda et al. 2011).

\subsubsection{The effects of primate feeding ecology on fruit characteristics}

Given the diverse and generally positive seed-dispersal services primates provide, it is not surprising that many fruit species strongly rely on primates for seed dispersal. In the Neotropics, for example, while fruits mainly dispersed by birds may also be consumed by several non-flying mammals, many fruits evolved thick protective husks inaccessible for most birds and bats and rely on primate dispersal almost exclusively (Janson 1983; Link and Stevenson 2004). When searching and choosing these fruits, primates may use their manual capacities to remove the husk and investigations from various sensory capacities - tactile, visual and olfactory - to detect the fruits and assess their quality (Dominy et al. 2006). Accordingly, under the Dispersal Syndrome Hypothesis framework (see 1.1.2), we may ask ourselves which selection pressures primate seed dispersal has generated on the characteristics of fruits they disperse? How primate behavior, feeding strategy and sensory capacities shaped the fruits that rely on their dispersal services? 
Primate-dispersed fruits tend to contain larger seeds (Howe 1986) which are also offered in fruits larger than those of bird or bat dispersed species (Janson 1983; Link and Stevenson 2004; Flörchinger et al. 2010). The ability to promote dissemination of large seeds is a boon in sunlightchallenging tropical environments (see 1.1.1) and it is thus not surprising that many plant species evolved to rely on primates and other non-flying mammals, even at the price of the need to produce larger fruits to accommodate their larger body masses (Herrera 2002). Primate-dispersed fruits also tend to be better protected (i.e. to have a thicker husk) than fruits dispersed by bats or birds (Janson 1983; Link and Stevenson 2004; Flörchinger et al. 2010). This is presumably a direct response to the complex manual capacities of primates (Torigoe 1985), which allows them to open fruits and in turn allows fruits to be better protected against antagonists while retaining the availability to their main dispersal vectors. Finally, nutrient content has been suggested to vary between fruits dispersed by different frugivore assemblages. Primate-dispersed fruits were predicted to contain lower amounts of lipids compared to fruits dispersed by bats or birds (Howe 1986). The few direct comparisons of primate and non-primate dispersed fruits published to date do not report significant differences in their nutrient content (Flörchinger et al. 2010) or minor differences in sugar and fiber content, which may in turn derive from abiotic factors (Voigt et al. 2004). Nonetheless, these two studies were conducted in the Paleotropics, where the primatebird dispersal syndromes are less distinct than in the Neotropics (cf. Janson 1983; Gautier-Hion et al. 1985).

Another fruit trait that may have been under selection pressures by seed-dispersing primates is color. Fruit color has been shown to have evolved in bird-dispersed fruits to mediate the interaction between birds and plants (see 1.1.2). Primates are the only eutherian mammals known to have evolved trichromatic vision (Jacobs 2009), and trichromacy is habitual among all cattarrhines and presents population level polymorphisms in which some females are trichromats whereas all males and the rest of the females are dichromats, in most platyrrhines and in some strepsirrhines (Dominy 2004a; Leonhardt et al. 2008; Jacobs 2009). Given that vision is considered the main sensory modality in primates (Fobes and King 1982) and that it is involved in every phase of their food acquisition process (Dominy et al. 2001, 2006), it becomes probable that fruits dispersed by 
primates would use color to communicate with them similarly to bird-dispersed fruits. Accordingly, the dominant view for the evolutionary forces leading to the evolution of primate trichromacy in primates is their frugivory - thus implying a coevolutionary process in which fruit color and primate color vision enhance each other (Regan et al. 2001).

Indeed, most primate dispersed fruits, at least in the Neotropics, change their color at ripeness (Regan et al. 2001) and become more conspicuous to foraging primates (Matsumoto et al. 2014; Melin et al. 2014). However, the color-vision-frugivory connection may not be so straightforward. First, regarding what primates can do with their color vision, as opposed to birds (see 1.1.2), there is no evidence that they can detect differences in fruit quality (i.e. nutrient content) based on fruit color (Dominy 2004a; Dominy and Lucas 2004). Accordingly, among polymorphic species, trichromatic individuals do not find fruits in higher rates (Dominy et al. 2003) or enjoy an increased caloric intake from fruits (Vogel et al. 2006). Second, regarding the evolution of fruit color, primate-

dispersed fruits provide visual signals that are less conspicuous than bird-dispersed fruits (Lomáscolo and Schaefer 2010), which implies that, all other things being equal, selection pressures exerted by the primate visual system on fruit color were weaker than those generated by birds' tetrachromacy. Finally, trichromacy proves to be more useful in folivory (Dominy and Lucas 2001, 2004). Thus, it is far from certain that primate color vision has evolved mainly in the context of frugivory, and therefore, fruit color in most species is probably not, or at least to a significantly lesser degree, an adaptation to signal ripeness to them similarly to the patterns we observe in bird-dispersed fruits. So, despite the supposed primacy of vision among the sense of primates, it appears to have had a minor effect on the evolution of fruit traits.

\subsubsection{Primate olfaction}

Olfaction - the sense of smell - has long been speculated, and recently confirmed for bat-fig interactions, to have exerted selection pressures on fruit traits, namely fruit odor (see 1.1.3). However, for long this speculation has been absent from the discussion of primates and the fruits 
they disperse. This is mainly due to the fact that primates have traditionally been viewed as "microsmats", whose sense of smell is reduced to practically negligible levels (Laska et al. 2000; Heymann 2006). This view was established in the early days of the study of primates (e.g. Elliot Smith 1927) and was based on the observation that primates have simpler nasal structures (Smith et al. 2007) and, relative to their brain size, small Main Olfactory Bulbs (MOB) - the brain structure dedicated to initial processing of olfactory stimuli (Baron et al. 1983; Ankel-Simons 2007). This has been viewed as a direct consequence of the elaboration of vision (Cartmill 1974) - a notion that is in line with the alleged increase in the centrality of vision among "higher" primates (Kirk and Kay 2004) and with the fact that the trends of reduction in nasal complexity and relative MOBs size are most apparent in the very same "higher" primates (Baron et al. 1983; Ankel-Simons 2007; Smith et al. 2007). Alongside our own experience as a vision-oriented species, the notion of "microsmatic primates" prevailed and the study of primate olfaction was almost absent for many decades.

First breaches in this paradigm came about when social functions of the sense of smell have been identified in several primate species (Michael et al. 1976) and eventually also in humans (Wysocki and Preti 2004). Yet the biggest blow to the notion of "microsmatic primates" came from a series of studies that, instead of speculating on primates' olfactory capacities based on anatomical proxies, measured actual performance in detection and discrimination tasks and demonstrated that, at least with regards to some compounds and compound classes, primate species of all main lineages outperform "macrosmats" such as dogs or rodents (Laska and Freyer 1997; Laska et al. 2000, 2005a,Laska and Seibt 2002a,b). These discoveries allowed to finally depart from the traditional notion that "olfaction doesn't matter" and led to a proliferation of studies that looked at the ecological functions of olfaction (Bolen and Green 1997; Bicca-Marques and Garber 2004; Phillips et al. 2004,Laska et al. 2007a; Siemers et al. 2007; Melin et al. 2009). However, these studies were often not integrated in a clearly defined framework, and were thus either not fully comparable or not controlling for all confounding factors. For example, several studies (e.g. Gilad et al. 2004; Dong et al. 2009) used counts of potentially functional olfactory receptor genes (ORs), which code the different receptors responsible for detection of volatile compounds, as a proxy for 
interspecific comparisons of "olfactory acuity". However, none tried to elucidate what actual physiological capacity a higher number of ORs may generate. Other studies (e.g. Siemers et al. 2007) performed controlled behavioral experiments without clear definitions of the actual function of the sense of smell in primates' feeding ecology, and found themselves demonstrating high performance in tasks that are ecologically irrelevant. As a result, even though the notion that "olfaction doesn't matter" shifted to "it may indeed matter", it is difficult to pinpoint large-scale evolutionary directions in primate olfaction: it is not clear how the sense of smell helps, if at all, to the process of food acquisition and thus which ecological niches generated selection pressures that promoted elaboration of different olfactory capacities. Consequently, apart from vague speculations (e.g. Howe 1986), primate olfaction has been absent from the study of seed dispersal and was ignored as a potential factor exerting selection pressures on fruit characteristics.

\subsection{Synthesis: the chemical ecology of primate seed dispersal}

The "chemical domain" in primate-plant interaction remains, therefore, unexplored. From the plants side of the interaction, the systematic investigation of fruit's employment of volatile compounds as a signaling mechanism to seed dispersers is limited to the narrow model system of bats and figs. From the primate side, it is by now accepted that the sense of smell may have an important role in their feeding ecology, but data are scarce and unintegrated and we do not know what primates actually do with their sense of smell in the process of food acquisition and in which species and contexts it is prominent. However, the data collected so far points at a clear

direction: if fruits are likely to have evolved to signal ripeness through olfactory cues, and if primates are important seed dispersers and have a good sense of smell, it becomes very likely that just like primate anatomy shaped fruit morphology, their sense of smell exerted selection pressures that shaped fruit chemical profiles.

The goal of this thesis is to explore this underinvestigated "chemical domain" of primate-plant interactions. The main hypothesis tested is that fruit odor in primate-dispersed species is an 
General introduction - Synthesis: the chemical ecology of primate seed dispersal

adaptation to primate seed dispersal, i.e. it has evolved in order to promote consumption of ripe fruits by primates and the dispersal of mature seeds. To test this hypothesis, both sides of the interaction - primates and plants - ought to be looked at. Thus, the thesis is comprised of three chapters that alternate between these two parties.

The first, still introductory, chapter $\mathbf{2}$ is a theoretical review that attempts to critically summarize and organize the available behavioral data regarding the use of olfaction in the process of primate food acquisition. Its goal is to examine the roles of the sense of smell in primate feeding ecology and determine in which contexts it becomes most important. More specifically, it asks two main questions:

a. What is the main function of olfaction in primates' feeding ecology?

b. Which ecological niches favor more reliance on the sense of smell?

Despite the scarcity of data, this introductory chapter concludes that frugivory is a context that often requires more reliance on olfaction and, more importantly, that the main function of olfaction in this context is food selection rather than detection. This means that primates are unlikely to rely on olfaction to locate feeding trees: they probably do so using their spatial cognition in the longer range and vision from a shorter range. Olfaction, in contrast, is used to determine, within a feeding tree, whether each individual fruit is ripe or unripe.

If this is indeed the case, it implies that the putative selection pressures exerted by the primate sense of smell on fruits would not act to generate particularly strong odors that could be carried away to long distances, but rather to emit unique odors that reliably mark ripe fruits and allow primates to know that an individual fruit is ripe and thus promote its ingestion and consequent seed dispersal. To address this, chapter 3 switches its focus to the plant side of the interaction. Its first goal is to examine whether fruit species whose main dispersal vector is primates bear an odor which is potentially informative of their ripeness level. So, its first question is:

c. Do fruits of primate-dispersed species emit a unique odor at ripeness, thus making their olfactory signature a reliable indicator for their ripeness level? 
Second, to examine whether this could be considered an adaptation for that function rather than a byproduct which is secondarily used by primates, the chapter employs a comparative approach. It compares the patterns of odor production in ripe and unripe fruits from four species: two primateand two bird-dispersed. Birds are considered to have much lesser reliance on olfaction and higher visual capacities. Thus, under the Dispersal Syndrome framework, they are expected to focus their signaling efforts on visual cues. By comparing to patterns of odor change at ripeness to birddispersed species, it allows asking the question:

d. Is the production of unique odor at ripeness a distinct feature of species whose main dispersal vector is primates (or other olfactory-developed frugivores), or does it also exist in species whose main dispersal vector is olfactory-less-dependent such as birds - and is thus an inevitable byproduct of fleshy-fruit maturation?

Further, the four plant species used as a model system correspond to a phylogeny in which each primate-dispersed species is phylogenetically closer to one bird-dispersed species than they are to one another. This allows to control for the effects of constraints on evolution, or phylogenetic inertia (Gould and Lewontin 1979), and ask:

e. Is the pattern of production of a unique odor at ripeness in primate-dispersed species independent of phylogeny?

The chapter demonstrates that fruits of primate-dispersed species indeed emit an odor unique to the ripe phase. This, however, does not yet prove that these odors have anything to do with the communication between primates and plants. To establish that, chapter 4 returns to the primate side of the interaction and reports bioassays conducted with captive monkeys that examines whether primates "understand" the signal putatively emitted by fruits. More specifically, it asks:

f. Can primates physiologically discriminate between the odors of ripe and unripe primatedispersed fruits?

g. If yes, do they use this capacity to select ripe fruits in the absence of other cues such as color, texture etc.? 
The chapter provides data that confirms that primates "understand" this signal, and thus provide the final support to the hypothesis that fruit odor is an adaptation to signal ripeness to seeddispersing primates. However, the odor of fruits is complex and composed of many different volatile compounds, some of which may be produced for other functions (Cipollini and Levey 1997). Identification of the components of odors that evolved as a direct response to selection pressures exerted by primate sensory ecology is not only a difficult task - it may be impossible, and it is in fact slightly naïve to think that the compounds composing fruits odor are fully independent from one another. Yet assuming that the main function of odor production in the context of signaling to seed dispersers is an enhancement of the perceived uniqueness of the ripe fruits (i.e. how different is the smell of ripe and unripe fruits in the eyes, or rather the nose, of the frugivore), a starting point in elucidating which compounds have evolved for this function would be to compare their relative contribution to the discrimination capacity of primates. Thus, chapter 0 asks:

h. Which compounds in the odor profiles of primate-dispersed fruits disproportionally contribute to primates' ability to identify them against unripe fruits?

To summarize, questions (a) and (b) examine what primates tend to do with their sense of smell in the context of food acquisition, and establish the predictions with regards to the selection pressures on fruit traits that are expected under the Dispersal Syndrome Hypothesis. Question (c) tests whether the volatile profile of primate-dispersed fruits is in accordance with these predictions, while questions (d) and (e) examine, through comparison to other species, whether this pattern is likely to be an adaptation evolved for this function. Returning to the primate side of the interaction, questions ( $f$ ) and $(g)$ ask whether primate physiological capacities and foodselection behavior correspond to the predictions that derive from the observed patterns of fruit odor release. Finally, question (h) addresses a more proximate level and asks which particular components of fruit odor can be attributed to the putative "odor as an evolved signaling mechanism" hypothesis tested here. 


\section{OLFACTION IN PRIMATE FEEDING ECOLOGY}

Led by the Nose: Olfaction in Primate Feeding Ecology

2015

Evolutionary Anthropology 24: 137-148

DOI: $10.1002 /$ evan.21458

With:

Eckhard W. Heymann, Behavioral Ecology and Sociobiology Unit, German Primate Center, Kellnerweg 4, 37077 Göttingen, Germany 


\section{$\underline{\text { Abstract }}$}

Olfaction - the sense of smell - was a latecomer to the systematic investigation of primate sensory ecology after long years in which it had been considered to be of minor importance (Heymann 2006). This view shifted with the growing understanding of its role in social behavior (Michael et al. 1976) and the accumulation of physiological studies which demonstrated that the olfactory abilities of some primates are on par with those of olfactory-dependent mammals such as dogs and rodents (Laska and Freyer 1997; Laska et al. 2000). Recent years have seen a proliferation of physiological, behavioral, anatomical and genetic investigations of primate olfaction that has begun shedding light on its importance in the process of food acquisition. However, integration of these works has been limited and it is therefore still difficult to pinpoint large-scale evolutionary scenarios, namely what functions the sense of smell fulfills in primates' feeding ecology and which ecological niches favor heavier reliance on olfaction. Here, we review available behavioral and physiological studies from the field or captivity, and try to elucidate how and when the sense of smell can help primates in the process of food acquisition. 


\subsection{Introduction}

The mammalian sense of smell is managed by several functionally distinct systems of which the major ones are the main and the accessory olfactory systems (Smith and Bhatnagar 2004). Although some overlap between the two exists (Baxi et al. 2006), the accessory olfactory system is generally dedicated to processing intraspecific social cues and signals (e.g. pheromones) whereas the main olfactory system deals with airborne chemicals from other sources (Lledo et al. 2005). Thus, chemosensation via the main olfactory system is what most of us would recognize as smelling: it allows sampling, detection and identification of volatile compounds from the environment (Fig. 2.1).

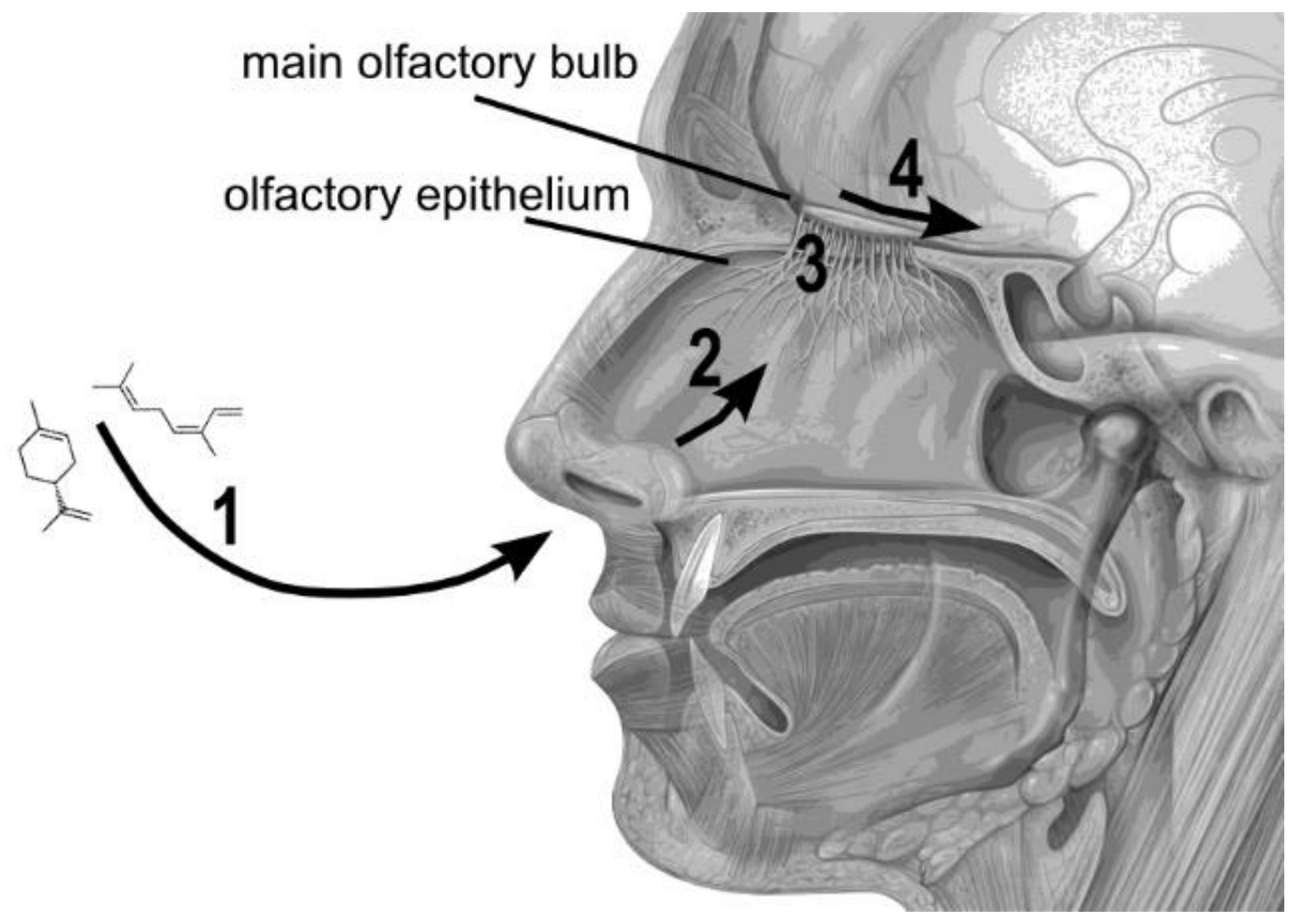

Figure 2.1. Smelling through the main olfactory system. (1) Airborne chemicals enter the nasal cavity when inhaling or when actively sniffing. (2) The odorants reach the olfactory epithelium, which hosts millions of nerve cells. Each nerve cell expresses only one type of olfactory receptor and projects to the main olfactory bulb - the first processing unit of the main olfactory system. (3) Odorants bind to only few olfactory receptors and evoke action potentials that are carried to the main olfactory bulb. (4) The signal from the one or more odorants is processed in the main olfactory bulb and then passed to the olfactory cortex, the limbic system and the rest of the brain (Mori et al. 1999; Buck 2004; Lledo et al. 2005). Figure adapted from an original by Patrick J. Lynch, medical illustrator; C. Carl Jaffe, MD, cardiologist; CC 2.5. 
There is, by now, little argument over the notion that primates have a good sense of smell. High olfactory ability entails two major olfactory capacities: olfactory sensitivity and discrimination capacity. Olfactory sensitivity is the ability to detect odorants at relatively low concentrations and discrimination capacity is the ability to perceive that two odors are different from one another and thus also to recognize odors. In a series of physiological studies, several primate species from different lineages have been shown to possess high olfactory sensitivity (Laska et al. 2000,Laska and Seibt 2002b) and discrimination capacity .(Laska and Freyer 1997,Laska et al. 2005a). Notably, their performance was on par with that of mammals which are considered to be highly olfactory-dependent such as dogs or rodents. These high olfactory capacities have been demonstrated to be relevant in various contexts, from social behavior (Palagi and Dapporto 2006) to predator avoidance (Sündermann et al. 2008). Not surprisingly, another context in which the sense of smell has been shown to be useful is food acquisition. Studies in the wild described "sniffing" behavior in foraging primates and many captive experiments have shown that primates of several lineages can use olfactory cues to detect or choose feeding items (Dominy et al. 2001, 2006). It thus became clear that the sense of smell is likely to play an important role in primate feeding ecology.

But beyond knowing that it may be important, how much do we know about what it actually does? In which contexts is it most informative? Which ecological niches favor higher reliance on olfaction and possibly select for higher olfactory sensitivity or discrimination capacity?

Previous attempts to answer some of these questions have focused on comparative analyses of anatomic and genetic components of the primate main olfactory system. Interspecific variation in several features of the main olfactory system has been documented: species differ in the surface area of the olfactory epithelium (Smith et al. 2004), the size of the main olfactory bulb (Stephan et al. 1981) and the number of functional olfactory receptor genes, which code the receptors expressed on the olfactory epithelium (Rouquier et al. 2000). Several studies interpreted interspecific variation in these traits as evidence that frugivorous and insectivorous diets, as well as a nocturnal activity time, are associated with superior olfactory capabilities. Another argued that the acquisition of trichromatic vision in primates has led to a reduction in olfactory capacities (Tab. 2.1). Yet the basic assumptions behind them are questionable and most importantly, it is unclear which olfactory capacities these measurements approximate and whether they are at all useful in assessing the level of reliance on olfaction in a species (Box 2.1, 2.2).

Thus, current conceptions regarding how and in which contexts olfaction plays a role in primate food acquisition are still primarily based on genetic and anatomical proxies, while behavioral works, when taken separately, often serve as no more than anecdotal support for the notion that the primate sense of smell can be useful. However, many behavioral studies have accumulated since the last reviews dealing with the roles of olfaction in primate feeding ecology (Dominy et al. 
$2001,2006)$ and addressing them together allows to begin to understand how the high olfactory capacities of primates translate into success in realistic ecological tasks in the context of food acquisition.

Here, we take a step in this direction and review available behavioral studies regarding the role(s) of olfaction in primate feeding ecology. Rather than simply describing all instances of usage of olfaction in the context of food acquisition, we organize relevant behavioral studies in a way that can help departure from the general notion that olfaction is useful and elucidate what primates do with their sense of smell when addressing feeding challenges, when it is useful and when it is not. We ask two main questions:

- What function the sense of smells fulfills in primate food acquisition?

- Which ecological niches favor higher reliance on olfaction?

The starting point for the first question is the distinction between the searching and selection phases (Dominy et al. 2006), i.e. the localization vs selection and quality assessment of food resources. These two levels may require different capacities and provide different kinds of information: "where food might be?" as opposed to the quality of individual items such as fruits on a tree that provides fruits of different degrees of ripeness. We then move on to the second question and examine two aspects of ecological niches that have been suggested to generate different requirements from the olfactory system: dietary strategies and the availability of visual cues. The goal is to use behavioral works and apply a (qualitative) comparative approach to examine whether species with different diets or those for whom visual cues are less available tend to use their sense of smell more than other primates when searching for or choosing food items. A concluding section emphasizes the difficulties presented by currently available data and offers directions for future studies.

\section{Box 1.1. Linking brain structures and olfactory performance}

Two main anatomical features of the main olfactory system have been considered to be good proxies to estimate the importance of olfaction in different species: the surface area of the olfactory epithelium, on which olfactory receptors are expressed, and the size of the main olfactory bulb (MOB), which projects to the olfactory epithelium and processes its input (Mori et al. 1999; Smith et al. 2004; Lledo et al. 2005). The surface area of the olfactory epithelium can potentially be indicative of olfactory sensitivity because it could host more olfactory receptors, thus allowing detection of odorants at lower concentrations (Smith et al. 2004). However, the fact that the number of receptors per unit of area is not constant across taxa renders it an unreliable 
measurement (Smith et al. 2004). The MOB, on the other hand, is clearly defined in the brain and, due to its direct projection to the olfactory epithelium, may present a better approximation for the actual number of olfactory receptors and thus olfactory sensitivity.

Volumetric data for the size of the MOB in dozens of primate species are available (Stephan et al. 1981) and have been used to compare different species and lineages (Baron et al. 1983; Barton et al. 1995; Barton 2006). But how should size be measured? Primates' brain size is strongly correlated with body mass (Jerison 1955) and individual brain structures change allometrically with brain size (Finlay and Darlington 1995). Thus, variation in MOB size that derives from differences in body mass is presumably not informative for any adaptationist discussion. This logic led comparative studies of primates (Baron et al. 1983; Barton et al. 1995; Barton 2006) and other taxa (Hutcheon et al. 2002) to use the relative rather than absolute size of the MOB. This approach, however, has been criticized. In contrast to total brain volume, which is assumed to grow with body mass to accommodate the increase in muscle mass and sensory input from a higher surface-area of the skin, olfactory information, or sensory demand, is not directly related to body size. It is therefore doubtful whether a larger animal would require a larger MOB (Smith and Bhatnagar 2004). Additionally, the MOB is not fully constrained by total brain volume and shows some degree of independent evolution (Barton and Harvey 2000; Finlay et al. 2001), and the total number of neurons in the primate main olfactory bulb is correlated with its absolute mass (Ribeiro et al. 2014). The size of the eyes or the primary visual cortex (V1) follows this same logic. Larger animals do not need larger eyes, and the absolute size of the visual cortex is correlated with the number of neurons and consequently with success in solving visual challenges between and within species (de Sousa and Proulx 2014). Thus, other studies used the absolute, and not relative, size of the MOB as a proxy for olfactory sensitivity (Heritage 2014).

Making things even more complex, when scaling aspects of the olfactory system to body or brain size, most studies log-transform the variables in order to meet the assumptions of the statistical methods employed (Baron et al. 1983; Barton et al. 1995; Barton 2006). Although this is a standard procedure, it might alter the conclusions drawn from the same dataset (Fig. 2.2). For example, before log-transformations, the nocturnal owl monkeys (Aotus spp.) have the largest $\mathrm{MOB}$ to brain ratio in all anthropoids. After transformation, they lag behind six other diurnal taxa (Stephan et al. 1981).

The question of proper scaling is not unique to the main olfactory system. For example, different legitimate measurements of brain size support different hypotheses regarding encephalization in primates (Deaner et al. 2000). This emphasizes that before using any measurement as a proxy for another trait, the functional and biological relations between the two must be fully understood. So, while it is plausible that there is some connection between the size of the MOB in a species and its olfactory capabilities, it is difficult at this point to draw any direct line between the two. 


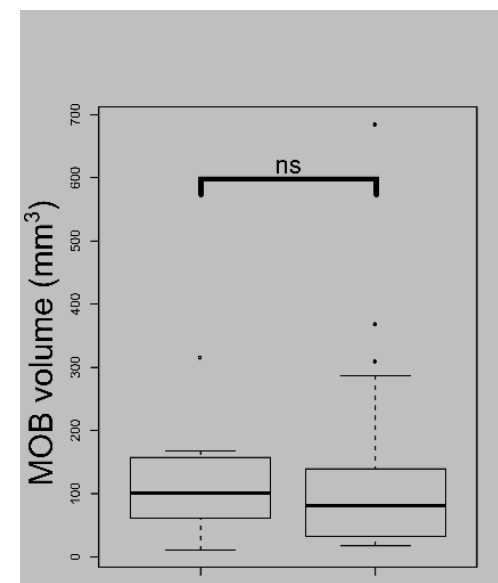

Fol

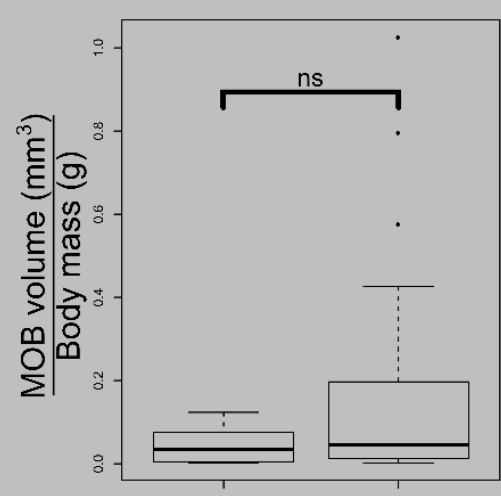

Fol

Fru/Fau

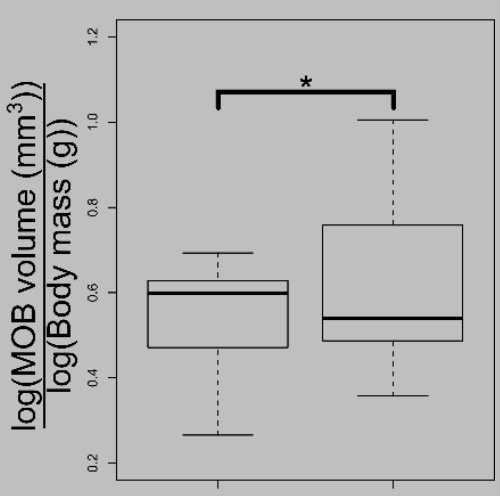

Fol

Fru/Fau

Figure 2.2. Same data, different conclusions. Differences in MOB (main olfactory bulb) size between folivorous (Fol) and frugivorous/faunivorous (Fru/Fau) primates using three legitimate scaling methods: absolute $\mathrm{MOB}$ volume, $\mathrm{MOB}$ volume relative to body mass and $\mathrm{MOB}$ volume relative to body mass when both variables are log-transformed. MOB volume and body mass data are from Stephan et al. (1981) $(\mathrm{N}=$ 36 frugivorous/faunivorous and $\mathrm{N}=8$ folivorous species) and dietary categories from Kappeler and Heymann (1996). Asterisk denotes significance at $\alpha=0.05$ from an independent contrasts linear regression model (Felsenstein 1985). Analyses were conducted on R 3.0.3 (R Core Team 2014) with package Caper (Orme et al. 2012) using an independent-rates soft-bounded constraints phylogeny from Springer et al. (2012). The absolute MOB size of folivorous primates is on average a bit larger but in a statistically non-significant level $(F=0.53(1,42), p=n s)$. This is probably because folivorous species tend to be larger (Terborgh 1992) and thus have larger brains and MOBs. The effect is reversed when looking at relative MOB size $(F=1.05$ (1, $42), p=n s)$ and becomes statistically significant only when data are log-transformed $(F=4.14(1,42), p=$ 0.048 ). This may reflect genuine higher emphasis on olfaction in these species, or merely an artifact of wrongly correcting for a larger body size in folivores.

\section{Box 2.2. The genetics of olfaction and comparative studies}

The Nobel winning discovery of the olfactory receptor (OR) gene family (Buck and Axel 1991) introduced molecular biology to the study of olfaction and vice versa. Members of this massive gene family code the majority of the different olfactory receptors of the main olfactory system. They are expressed on the olfactory epithelium, and a broader range is assumed to be associated with the ability to detect more compounds or better discriminate between odors (Young and Trask 2002; Nei et al. 2008). Since some OR genes have lost functionality in all primate lineages ("pseudogenization"), the remaining number of intact genes, or the proportion of pseudogenes, has been used to infer inter-specific variation in olfactory discrimination capacity (Rouquier et al. 2000; Gilad et al. 2004; Zhang et al. 2007; Dong et al. 2009; Matsui et al. 2010). This assumption is partially supported by physiological studies (Rizvanovic et al. 2013) and is rather sound because 
animals like rodents, which are generally considered as highly olfactory-dependent, tend to have substantially more intact ORs than animals such as dolphins, which are considered to have little or no reliance on olfaction (Niimura 2012).

It comes, however, with some caveats. To begin with, potentially functional ORs can be expressed in non-olfactory tissues while pseudogenes can be expressed in the olfactory epithelium (Zhang et al. 2007). Thus, the number of intact OR genes is not necessarily equivalent to the number of expressed functional receptors in the main olfactory system. Moreover, even a good estimation of the actual number of functional receptors in the main olfactory system would only be a very crude proxy for the ability to rely on olfaction to address real-life challenges. The primate OR family has gone through a birth-and-death process that included positive and purifying selection (Gilad et al. 2005; Dong et al. 2009). This means that the functional genes in a given species are not simply a fraction of the ancestral stock but a unique combination of genes that may be well adapted to species-specific ecological requirements. For example, a hypothetical dietary specialist may rely on olfaction more than a generalist and present superior sensitivity and discrimination capacities in its respective niche, but possess a smaller total number of functional OR genes because its olfactory system has to deal with less diverse stimuli. Lastly, given the combinatorial nature of olfaction, even a relatively modest set of about 400 intact ORs present in humans allows the detection and discrimination of over 400,000 different volatile compounds (Mori and Manabe 2014) and of up to $10^{12}$ different odor mixtures (Bushdid et al. 2014). It is therefore hard to argue that this comparatively small number of functional ORs is necessarily associated with a handicapped sense of smell (Weiss 2014).

Thus, ignoring results from behavioral works and relying only on genetics has led to unwarranted conclusions. For example, in one of the more insightful genetic works to date, the authors note that the number of functional OR genes is similar in different haplorrhine lineages and thus cannot account for "the reduced olfactory ability in apes and [Old World monkeys]" (Dong et al. 2009, p. 263). This alleged reduced ability, however, has never been demonstrated in any physiological or behavioral test and its validity is based only on the not-fully established assumption that a relatively smaller main olfactory bulb is associated with a reduction in olfaction (Box 2.1).

The genetics of olfaction clearly has a potential for going beyond low-resolution measurements such as "discrimination capacity" and telling us which species is adapted to work with which olfactory stimuli, and so to what extent a species' main olfactory system has adapted to cope with the relevant ecological challenges. This, however, requires much more than counts of intact genes. It would not only require identifying the ecologically relevant odors, but also knowing which genetic makeup generates higher sensitivity to these stimuli and better discrimination between them. Although this approach is beginning to be established (Touhara 2014), we are still far from 
understanding the environment-olfaction interaction in such resolution for any species, let alone for primates.

\subsection{Olfaction: what is it good for?}

Food acquisition is a multi-step process that includes locating food and assessing its quality (Dominy et al. 2001, 2006). Olfaction can potentially be useful in both: over the usually longer distance to detect food items and over the shorter distances for quality assessment and selection of individual items (Dominy et al. 2006). Information from these two phases does not fully overlap. For example, a high quality fruiting tree may still have many undesired unripe fruits. Thus, finding it and identifying the ripe fruits are completely different tasks that may challenge primates in different ways.

\subsubsection{Food detection}

Olfactory guided long-distance food detection is probably the more challenging task and requires not only the ability to detect and identify the cue, but also to follow the odor and track its source. In macroscopic scales, air movement over an odor source creates an odor plume, similarly to smoke issued from a chimney. Odor plumes have a weak chemical gradient across large distances and therefore maintain the integrity, i.e. proportion of odorants, over long distances in a rather narrow plume. This allows animals to scent-track the origin of the plume, usually by meandering in and around it (Vickers 2000). Humans have been shown to be able to scent-track in two-dimensional open-field conditions where the trail was restricted to the ground and movement free (Porter et al. 2007). Yet odor plumes are carried in the air and as opposed to insects, which can meander around an odor plume, primate movement in the three-dimensional space is restricted. Thus, olfactory-based long-distance location of feeding sources is a challenge in which primates are not expected to excel.

Indeed, behavioral evidence for the ability of primates to use their sense of smell to find food over large distances is scarce. Two studies tested the ability of several wild and semi-wild New World primates to detect fruit-baited feeding platforms based on olfactory cues (Bolen and Green 1997; Bicca-Marques and Garber 2004). Owl monkeys (Aotus spp.) and one out of two emperor tamarin (Saguinus imperator) groups were capable of detecting the baited platform above chance level using only olfactory cues. However, these were the exceptions. Titi monkeys (Callicebus cupreus), tufted capuchins (Cebus apella), saddleback tamarins (Saguinus fuscicollis) and the second 
emperor tamarin group failed this task. Moreover, the task was to detect baited platforms from a distance of only few meters, which is easier than the distances over which most primates search for fruits.

A more recent study reported the failure of captive owl monkeys to locate baited feeding boxes based on olfactory cues (da Costa and Bicca-Marques 2014). The authors suggested that issues such as motivation and experimental design may have contributed to the negative results. Yet it still indicates that tracking food sources using olfactory cues is a task in which even owl monkeys, which perform better than other New World species in similar tasks (Bolen and Green 1997; BiccaMarques and Garber 2004), may not excel. Apart from that, neither positive nor negative evidence for long-distance olfactory foraging is available. The absence of evidence is not necessarily evidence of absence, but the fact that the typical and easily identified scent-tracking behavior most of us would identify from dogs is practically absent from the primate literature suggests that this may simply not be a part of their normal feeding strategy.

Successful use of olfaction to detect food has been implied only in shorter distances, when tracking odor plumes is not required. Slender lorises (Loris lydekkerianus lydekkerianus) increase the level of olfactory investigation when foraging for invertebrates (Nekaris 2005) and mouse lemurs (Microcebus murinus) can detect insect prey using only their sense of smell (Piep et al. 2008). In addition, short-distance olfactory guided foraging has been suggested to allow diademed sifakas (Propithecus diadema) to locate inflorescences hidden in the leaf litter (Irwin et al. 2007).

\subsubsection{Food selection}

Olfactory-based selection or assessment of individual items is probably an easier task that would usually be conducted at a very short distance in combination with other senses (Dominy et al. 2006). Here, the challenge is to discriminate between the odors of, for example, ripe and unripe fruits and choose accordingly. Indeed, evidence for the importance of olfaction to primate food selection is much more abundant.

Several studies reported an increase in sniffing behavior in situations that require quality assessment of feeding items (Fig. 2.3). As opposed to passive inhaling, sniffing is an active process of sampling to the main olfactory system in which repetitive sampling in differing speeds, volumes and frequencies increases the ability of the animal to assess odorant identity and concentration (Mainland and Sobel 2006; Verhagen et al. 2007). Thus, elevated levels of sniffing are indicative of more thorough olfactory investigation and reliance on olfactory cues. Sniffing of feeding items from short distances has been described in captivity (Zschoke and Thomsen 2014) 
and in the wild (van Roosmalen 1985b; Matsumoto-Oda et al. 2007). Since sniffing occurs after the item is located, it clearly fulfills the function of quality assessment. Applying a more quantitative approach, two studies on wild spider monkeys (Ateles geoffroyi) and white-faced capuchins (Cebus capucinus) examined patterns of sniffing behavior when selecting figs (Hiramatsu et al. 2009; Melin et al. 2009). Both species increased their sniffing behavior when feeding on fig species that are visually cryptic - i.e. do not provide a reliable visual cue for ripeness. This demonstrates the importance of olfaction for fruit selection after their location. Additionally, Experiments held in captivity showed that tufted capuchins increase the rate sniffing rate when unpalatable secondary compounds (pepper) are added to otherwise desired food (Visalberghi and Addessi 2000) and that both spider monkeys and squirrel monkeys (Saimiri sciureus) increase frequency of sniffing when facing novel items, probably in an attempt to estimate their quality (Laska et al. 2007c). So, evidence from the wild and captivity indicates that sniffing - active, repetitive olfactory sampling - is common when examining feeding items from close proximity and increases when facing novel items or when visual cues are unavailable.

Furthermore, few works examined experimentally whether primates can rely on olfactory cues to assess the quality of feeding items. In captivity, three lemur species (ruffed lemurs - Varecia variegata, Coquerel's sifakas - Propithecus coquereli, ring-tailed lemurs - Lemur catta) were challenged to discriminate between food items under two conditions: preferred red (ripe fruits or young leaves) and less-preferred green (unripe fruits and mature leaves) (Rushmore et al. 2012). Olfactory cues, either alone or in combination with visual cues, aided all three species to correctly choose the desired items. Our own work suggests that both cotton-top tamarins (Saguinus oedipus) and common marmosets (Callithrix jacchus) can quickly learn (within $1 \mathrm{~h}$ ) to discriminate between random novel odors marking positive and negative rewards and use them to select desired odorless rewards (Rathke \& Nevo, unpub. data). Finally, based on discrimination between positive (food-related) and negative stimuli, captive pigtailed macaques (Macaca nemestrina) learn to achieve high success rates in foraging tasks (Hübener and Laska 1998). 

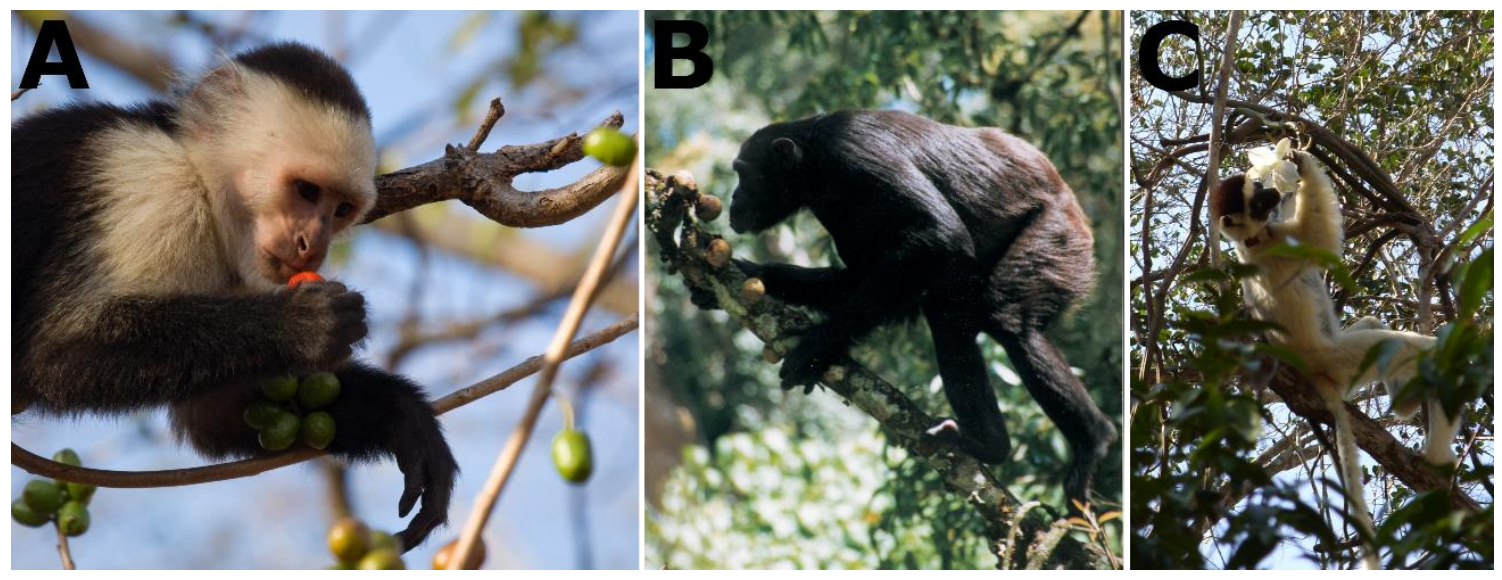

Figure 2.3. Sniffing of feeding items. (A) In the final stage of quality assessment, a white-faced capuchin in Área de Conservación Guanacaste, Costa Rica, sniffs a ripe fruit of Spondias purpurea after its removal (photo: Fernando Campos). (B) A chimpanzee sniffing fruits of Ficus bracylepis in Kibale National Park, Uganda (photo: Nathaniel J. Dominy). (C) A verreaux's sifaka sniffs a flower of Vanilla madagascariensis, which they occasionally consume (Andrea Springer, pers. comm.) in the Kirindy Forest, Madagascar. Although sniffing of ingested flowers in primates has not been systematically recorded, it is likely that olfactory cues are involved in the selection process (photo: Andrea Springer).

\subsubsection{Summary: the function of olfaction in food acquisition}

Olfaction can potentially serve two separate functions in primate feeding ecology: food detection and selection. In fruit foraging, detection is usually conducted over long distances and evidence for the use of olfaction in this process are scarce to non-existent. In contrast, there is much more evidence, from the wild and from captivity, for reliance on olfaction for quality assessment of feeding items. Olfaction can be useful for locating feeding items only when olfactory sensation takes place over very short distances that do not require tracking odor plumes. For example, insectivores may use it to determine whether tree-holes contain prey. However, in this case the use of olfaction is functionally very similar to selection. Olfaction may also be used for quality assessment of caught invertebrates, but we are not aware of any study that tested that.

An earlier account of primate sensory ecology (Dominy et al. 2001) schematically portrayed the different "sensory boundaries" of primate food perception - i.e. the distances from which the foraging primate can gather information through several sensory trajectories. While acknowledging that evidence for the use of olfaction over long distances had been scarce, it still 
placed the olfactory boundary far away from the foraging primate, somewhere between the visual and auditory boundaries. Given the data that have accumulated since, we believe that the olfactory boundary should be pushed back and considered to be almost as small as the tactile boundary. As important as olfaction may be, current evidence suggest that it serves primates mainly at very short distances.

\subsection{Which ecological niches favor reliance on olfaction?}

So olfaction could play an important role in primate feeding ecology, even if primarily from short distances, and there are examples for reliance on olfaction to acquire food in many contexts: when feeding on fruits or invertebrates, at day or night and by representatives of all major lineages. However, there is a good reason to believe that its importance - i.e. the fitness benefit from the ability to acquire olfactory information quickly and accurately - is not constant across the diverse ecological niches which primates occupy. Variation in the relative size of the main olfactory bulb and the share of functional olfactory receptor genes has been interpreted as evidence for differences in the importance of olfaction across these ecological niches (Tab. 2.1), but the validity of these proxies is questionable (Box 2.1, 2.2).

Here, we examine whether available behavioral data indicate that olfaction is more important in some ecological niches than it is in others. We look at studies that quantified sniffing behavior and directly tested success rates in foraging tasks that represent superior olfactory sensitivity or discrimination capacity. The relationship between the two is bidirectional: ecological contexts in which olfactory cues can provide more useful information, or those in which cues from other trajectories are less available, are expected to increase the level of reliance on olfaction. This should manifest in increased olfactory sampling of feeding items, i.e. sniffing. Over evolutionary time, a likely scenario is that species that show higher dependence on olfactory cues would be subjected to selection pressures for elaboration of olfactory sensitivity and discrimination capacity. Superior ability to exploit olfactory cues should lead, in turn, to higher allocation of time to olfactory investigation during the food-selection processes, and thus elevated sniffing behavior. 


\subsubsection{Dietary strategies}

Olfaction may play a role foraging for practically all primates' dietary categories. Fleshy fruits often bear odors that are unique to their ripe phase and may have even evolved to be olfactoryconspicuous to frugivores (Hodgkison et al. 2007, 2013). Further, fruit volatile compounds may be indicative of their nutrient content (Goff and Klee 2006) and mouse lemurs have been reported to consume fruit species that are more odorous than other sympatric species (Valenta et al. 2013). Olfaction may be useful for in other categories as well. The volatile signatures of broken young and mature leaves are different (Kant et al. 2009) and invertebrates sometimes emit odors that may allow their identification (Hilker and McNeil 2008). However, choosing these items or searching them over short distances also involve acquiring information from other sensory trajectories: auditory cues when catching prey, tactile cues when choosing fruits or leaves and visual cues in practically all. Additionally, young leaves tend to grow in predictable locations on the branch (Fleming 2005), which may provide a "micro-spatial" cue. Thus, olfactory cues may be very useful in some cases and redundant in others, and the questions asked here is whether feeding on items of some dietary categories is associated with more reliance on the sense of smell than others.

Although only few studies allow addressing this question, those available indicate that olfaction is particularly useful when feeding on fruits. Here, too, evidence comes from studies that quantified the frequency of sniffing behavior, i.e. the tendency to sample the odor of feeding items, and from more direct interspecific comparisons of success rates in foraging tasks. In controlled experiments conducted in captivity, spider monkeys were found to increase their sniffing behavior more than squirrel monkeys when addressing novel feeding items (Laska et al. 2007c). This was explained by spider monkeys' higher degree of frugivory as opposed to the more insectivorous squirrel monkeys, thus indicating that more frugivorous species tend to rely more on their sense of smell. Another study compared the tendency to rely on olfactory cues (sniff) as opposed to visual cues in discrimination tasks in three captive Malagasy primates of different dietary categories. 
Frugivorous ruffed lemurs showed the highest tendency to sniff items and could solve the task only when olfactory cues were present. Folivorous Coquerel's sifakas showed a lower level of sniffing behavior and could not solve the task based solely on olfactory cues. Ring-tailed lemurs, as generalists, were intermediate. They tended to use more visual than olfactory investigation, but could solve the task using either of them alone (Rushmore et al. 2012). Taken together, these studies indicate that more frugivorous species have a higher tendency to sniff feeding items while species that show a lesser degree of frugivory are less likely to employ olfactory investigation and rely more on visual cues.

In another set of studies, mouse lemurs have been shown to be able to detect both fruits and invertebrate prey hidden under opaque lids based solely olfactory cues, but success rates were much higher for fruits and approximated 100\% (Siemers et al. 2007; Piep et al. 2008; Siemers 2013). Capuchin monkeys, who were reported to employ their sense of smell when choosing fruits (Melin et al. 2009), fail to locate invertebrates from close proximity when only olfactory cues are available in captive conditions (Phillips et al. 2004). Capuchins were also shown to have higher olfactory discrimination capacity for fruity (as opposed to fishy) odors, thus indicating that their sense of smell is particularly tuned to fruity stimuli (Ueno 1994). Thus, species that consume both fruits and invertebrates achieve higher success rates in foraging tasks when using olfaction for fruit foraging. Finally, physiological studies in several primate species reported high sensitivity to and discrimination of odorants commonly present in fruits (Hernandez Salazar et al. 2003,Laska et al. 2006a,b). Whether or not the primate sense of smell is similarly tuned to odorants emitted by invertebrates or leaves is unknown.

So, although comparative behavioral data are still based on a small number of species, they indicate that olfaction is more important in fruit selection than it is in foraging for invertebrates or leaves. This may derive from differences in the difficulty of using olfaction in these tasks. In fruit foraging, as noted above, the main function of olfaction is not to locate a fruit but to determine whether it is ripe or not. Fruit odor can be endlessly re-sampled (sniffed) from a very short distance and may have even evolved to be olfactory conspicuous - i.e. to provide a reliable odor signal for 
ripeness (Hodgkison et al. 2007; Borges et al. 2008). Thus, when choosing fruits primates can extract more information via the olfactory trajectory and species for whom fruits constitute a larger share of the diet may have evolved superior olfactory capabilities. This, in turn, makes frugivorous species more likely to resort to reliance on olfactory cues and show elevated levels of sniffing behavior. Invertebrates, on the other hand, are expected to be under selection pressures to reduce their olfactory signature and even though there are descriptions of reliance on olfaction when finding them over short-distances, visual and auditory cues probably play a bigger role in their location. Finally, there are not even anecdotal reports of sniffing behavior in leaf foraging, thus suggesting that folivores may rely even less on olfactory cues when selecting leaves. The most likely explanation is that other cues - namely the location, texture and possibly color of the leaves - provide sufficient sensory information.

\subsubsection{Availability of visual cues}

The importance of olfaction for food acquisition depends, then, on the information given by the target's volatile signature and also by the potential of acquiring similar information through other sensory trajectories, thus rendering olfaction redundant. Vision is often considered to be the main primate sensory modality (Fobes and King 1982) and it is likely that the availability of visual cues reduces the importance of olfaction, and that by extension species whose visual capacities are higher would tend to rely less on their sense of smell, i.e. they will show reduced levels of sniffing behavior and, over time, may evolve to possess less acute olfactory sensitivity or discrimination capacity due to relaxation of selection pressures. The notion of a tradeoff between olfaction and vision in primates goes back to the earliest stages of biological anthropology (Elliot Smith 1927) and has been in the heart of the hypothesis that primates evolved as "visual predators" in which the enlargement of the orbitals forced a reduction of the olfactory apparatus (Cartmill 1974). It is further supported by the fact that primate olfactory and visual brain structures are negatively correlated (Barton et al. 1995). Here, we ask whether two factors that determine differences in the 
availability of visual cues - activity time and variation in color-vision capacities - predict differences in the level of reliance on olfaction.

\section{Activity time}

Among anthropoids, the prediction that the nocturnal owl monkeys tend to rely on olfaction more than diurnal counterparts (Wright 1989) has been confirmed in two independent studies (Bolen and Green 1997; Bicca-Marques and Garber 2004). Both employed similar experimental approaches and demonstrated that owl monkeys perform better in olfactory-based food-detection tasks than their diurnal counterparts. Yet since these results are based on a single species, to show that this is indeed a pattern they need to be replicated in strepsirrhines, in which more nocturnal species are available. Another interesting parallel would be a comparison of day and night feeding in cathemeral species or in diurnal species such as spider and woolly monkeys (Lagothrix spp.), which occasionally forage at night (Carlos Peres, pers. comm.).

\section{Color vision}

Primates are the only eutherian mammals known to have acquired full trichromatic vision (Jacobs 2009). However, not all species are fully trichromatic. Old World monkeys, apes and New World howler monkeys (Alouatta spp.) are habitually trichromatic, whereas all other species are di- or monochromatic, or present population-level polymorphism in which all males and some females are dichromats and the rest of the females are trichromats (Leonhardt et al. 2008; Jacobs 2009).

The prediction that a full trichromatic vision is associated with a reduction in olfaction has been mainly addressed in genetic works (Tab. 2.1). Two behavioral studies measured the tendency to sniff fruits before ingestion in wild groups of two New World monkey species. They genotyped the females to determine whether they were di- or trichromatic and tested the hypothesis that dichromats sniff fruits more often to compensate for the lack of visual information available to 
trichromatic group members. Results were contradictory: an effect was found in capuchins (Melin et al. 2009) but not in spider monkeys (Hiramatsu et al. 2009).

Evidence from comparative physiological studies provides a clearer picture. In a series of studies, M. Laska and colleagues compared olfactory sensitivity and discrimination capacity in three New and Old World monkey species: pigtailed macaques, squirrel monkeys and spider monkeys (Laska and Seibt 2002a,b,Laska et al. 2005a,b, 2006a, 2007b). Although not addressing the question directly, these experiments included male New World monkeys which are necessarily dichromats, female New World monkeys that may have been trichromats and Old World monkeys, which are trichromats. The results, encompassing a wide range of odorants, did not reveal any clear difference between New and Old World species and therefore imply that differences in color vision are not necessarily associated with interspecific differences in olfactory sensitivity or discrimination capacity.

Thus, the prediction of lesser reliance on olfaction by trichromats is, to this point, not unequivocally supported by available data. Although based on only few studies, if the lack of a trade-off between full color vision and olfaction is indeed genuine, two non-mutually exclusive explanations come to mind. The first is that acquisition of trichromatic vision does not impose strong constraints on olfaction. Trichromacy was achieved through a duplication of the M-L opsin gene (Jacobs 2009) and the expression of three rather than two opsin genes in the eye should not impose any constraints on olfaction like those attributed to the enlargement of the orbitals, which supposedly forced a reduction in olfaction (Cartmill 1974). A second, functional, potential explanation is that there is little overlap in the information that color vision and the sense of smell provide. Trichromatic vision has been speculated to specifically help in long-distance detection of fruits (Melin et al. 2014). Thus, if the sense of smell is indeed less important in anything beyond the short distance, color vision and olfaction may be complementary: trichromacy helps identifying fruit-bearing trees and olfaction is used in the short distance, along with other senses, to determine whether an individual fruit is ripe. 
Table 2.1 - Which ecological niches favor reliance on olfaction in primate feeding ecology? Summary of the behavioral evidence for differences in the level of reliance on olfaction when feeding on different dietary categories, between diurnal and nocturnal species and between tri- and dichromats, and a comparison to the conclusions from anatomical and genetic analyses. For the issues in interpreting the variation in anatomical and genetic features of the main olfactory system, see Box 2.1, 2.2.

\begin{tabular}{|c|c|c|c|c|c|c|}
\hline \multirow[b]{2}{*}{ Diet } & \multirow[b]{2}{*}{$\begin{array}{l}\text { Behavior } \\
\text { Primates rely on } \\
\text { olfactory cues } \\
\text { more when eating } \\
\text { fruits. Folivory } \\
\text { requires the least } \\
\text { reliance on } \\
\text { olfaction. }\end{array}$} & \multirow[b]{2}{*}{$\begin{array}{l}\text { Ueno } \\
1994 ; \\
\text { Phillips et } \\
\text { al. 2004; } \\
\text { Laska et } \\
\text { al. 2007c; } \\
\text { Siemers } \\
\text { et al. } \\
\text { 2007; } \\
\text { Piep et al. } \\
2008 ; \\
\text { Rushmore } \\
\text { et al. } \\
2012 ; \\
\text { Siemers } \\
2013\end{array}$} & \multicolumn{2}{|c|}{$\begin{array}{l}\text { Anatomy (main } \\
\text { bulb size) }\end{array}$} & \multicolumn{2}{|c|}{$\begin{array}{l}\text { Genetics (olfactory } \\
\text { receptor repertoire) }\end{array}$} \\
\hline & & & $\begin{array}{l}\text { Frugivores/insectiv } \\
\text { ores have a } \\
\text { relatively larger } \\
\text { main olfactory } \\
\text { bulb }\end{array}$ & $\begin{array}{l}\text { Baron et } \\
\text { al. 1983; } \\
\text { Barton et } \\
\text { al. 1995; } \\
\text { Barton } \\
2006\end{array}$ & $\begin{array}{l}\text { No clear } \\
\text { difference }\end{array}$ & $\begin{array}{l}\text { Gilad et } \\
\text { al. } 2004 \text {, } \\
2007\end{array}$ \\
\hline $\begin{array}{l}\text { Activity } \\
\text { time }\end{array}$ & $\begin{array}{l}\text { Nocturnal owl } \\
\text { monkeys } \\
\text { outperform diurnal } \\
\text { NWM in food } \\
\text { searching tasks }\end{array}$ & $\begin{array}{l}\text { Bolen and } \\
\text { Green } \\
1997 ; \\
\text { Bicca- } \\
\text { Marques } \\
\text { and } \\
\text { Garber } \\
2004\end{array}$ & $\begin{array}{l}\text { Nocturnal species } \\
\text { have a relatively } \\
\text { larger main } \\
\text { olfactory bulb }\end{array}$ & $\begin{array}{l}\text { Baron et } \\
\text { al. 1983; } \\
\text { Barton et } \\
\text { al. 1995; } \\
\text { Barton } \\
2006\end{array}$ & $\begin{array}{l}\text { No difference (but } \\
\text { based on one } \\
\text { species) }\end{array}$ & $\begin{array}{l}\text { Gilad et } \\
\text { al. } 2004, \\
2007\end{array}$ \\
\hline \multirow[t]{2}{*}{$\begin{array}{l}\text { Color } \\
\text { vision }\end{array}$} & $\begin{array}{l}\text { No clear } \\
\text { differences in } \\
\text { olfactory } \\
\text { sensitivity or } \\
\text { discrimination } \\
\text { between Old } \\
\text { World and New } \\
\text { World monkeys }\end{array}$ & $\begin{array}{l}\text { Laska and } \\
\text { Seibt } \\
2002 a, b ; \\
\text { Laska et } \\
\text { al. } \\
2005 a, b \text {, } \\
2006 a \text {, } \\
2007 b\end{array}$ & Not directly tested & & $\begin{array}{l}\text { No connection } \\
\text { between } \\
\text { trichromacy and } \\
\text { loss of functional } \\
\text { olfactory receptors }\end{array}$ & $\begin{array}{l}\text { Matsui et } \\
\text { al. } 2010\end{array}$ \\
\hline & $\begin{array}{l}\text { Within species: } \\
\text { mixed results }\end{array}$ & $\begin{array}{l}\text { Hiramatsu } \\
\text { et al. } \\
2009 ; \\
\text { Melin et } \\
\text { al. } 2009\end{array}$ & & & $\begin{array}{l}\text { Howler monkeys } \\
\text { have more } \\
\text { olfactory receptor } \\
\text { pseudogenes }\end{array}$ & $\begin{array}{l}\text { Gilad et } \\
\text { al. } 2004 \text {, } \\
2007\end{array}$ \\
\hline
\end{tabular}

\subsection{What next?}

The proliferation of behavioral and physiological work in the recent years has begun to establish trends that allow us to depart from the vague notion that olfaction may be important to primate feeding ecology and to begin to understand how it helps primates to acquire food and in which contexts it becomes useful. Currently available data indicate that olfaction is used primarily in 
short distances and usually for food selection, and support arguments for some of the factors that have been suggested to affect the level of reliance on olfaction (diet, activity period) but less for others (color vision). However, these data are still too scarce and unstandardized to reach clear conclusions. We are therefore hopeful that emphasizing these gaps in the available data will encourage future studies. More specifically, we see three main approaches that would significantly improve our understanding of the roles of olfaction in primate feeding ecology: (1) chemical ecology of primate feeding behavior: connecting primate physiological capacities and ecologically relevant chemically resolved stimuli; (2) a systematic comparative approach in behavioral studies to control for the many confounding factors; (3) identification of similar and contrasting trends in non-primate taxa.

\subsubsection{Chemical ecology of primate feeding behavior}

Something almost fully missing from available studies is an understanding of the chemical world with which the primate olfactory system has to deal in foraging and food selection. There are practically no published analyses of the odors of fruits primates consume or invertebrates they catch in the wild. So far, to our knowledge, only one study conducted chemical analyses of natural odors of fruits consumed by primates (Valenta et al. 2013), but even there the actual chemical profiles of primate-consumed fruits have not been published.

Modern techniques allow relatively easy and cheap sampling and analysis of volatile compounds in the field (Kalko and Ayasse 2009) and introducing them to the study of primate feeding ecology could resolve many questions. If indeed olfaction is more relied upon in fruit foraging and in some contexts in insect foraging, the targets for chemical investigation should be the odor profiles of ripe, unripe and overripe fruits, and occupied and non-occupied tree holes or other microhabitats in which prey can be found. This can, first, establish whether there is even a potential for reliance on olfactory cues: if an unripe fruit smells just like a ripe one, there is no need to look further. Once the "olfactory distance" between desired and undesired items is understood, many questions can be asked. Can primates discriminate between the odors? Are success rates in choosing the right 
fruit or tree hole greater when the odor is present? Is this ability learned or innate? Further investigations can focus on individual compounds or compound classes and see which contribute to primates' discrimination capacity. Finally, if certain compounds or compound classes turn out to be more important than others, functional genomics may be able to identify the olfactory receptors that allow their detection. Several studies have identified primate olfactory receptor genes that have gone through positive selection (Gilad et al. 2005; Dong et al. 2009). Thus, by connecting the two, future work may be able to reveal in great detail which chemical stimuli are relevant enough to have exerted significant selection pressures on the primate olfactory receptor gene repertoire. This is a trail that can lead to very high-resolution understanding of lineagespecific olfactory adaptations.

A more comprehensive knowledge of the olfactory challenges faced by primates can then be taken to the lab and applied in controlled captive experiments that would connect physiological capacities and the ability to deal with ecologically relevant challenges. So far, there has been almost a complete separation: studies have been either about physiology but less ecologically relevant, or vice versa. For example, available data suggest that in fruit foraging the main function of olfaction is fruit selection, but it is unclear which aspects of the primate olfactory system have been under positive selection due to this requirement. By measuring olfactory sensitivity and discrimination capacity of ecologically relevant and chemically known stimuli, comparative analyses can test whether more frugivorous species show higher sensitivity to the odorants emitted by fruits or higher discrimination capacity between these odorants and the odors of unripe fruits. When choosing fruits the most important information is not whether the fruit is there, but whether it is ripe, unripe, overripe or potentially infested. Considering that fruits are not expected to be under selection pressures to lower their olfactory signature, olfactory discrimination capacity is expected to be more important than sensitivity, and thus it can be predicted that the degree of frugivory in a species is positively correlated with discrimination capacity of relevant odorants.

A similar approach can be applied to location of slow or sedentary invertebrates from short distances. Here, the information sought after is mostly whether the prey is present or not. 
Furthermore, if primates and other predators use olfactory cues to locate these slow sedentary invertebrates, they are expected to be under selection pressures to lower their olfactory signature. Thus, it may turn out that olfactory sensitivity, and not discrimination, is the olfactory capacity most important for insectivores.

So, comparison of olfactory capacities when dealing with ecologically relevant stimuli could help departure from a rather simplistic discussion regarding "olfactory elaboration": olfaction can be useful in more than one way and different ecological challenges are expected to exert different selection pressures on the various aspects of the main olfactory system. Moreover, establishing the connection between an ecological challenge and olfactory capacity could be used to reassess the quality of measurements such as the size of the main olfactory bulb and the olfactory receptor gene repertoire as proxies for actual olfactory performance in species-specific ecological challenges.

\subsubsection{Comparative behavioral tests}

The main issue in the search of olfactory adaptation is the many confounding factors. For example, howler monkeys, as the only New World primate with routine trichromacy (Jacobs 2009), can be an interesting model taxon for examining whether elaboration of color vision is associated with a reduction in olfaction (Gilad et al. 2004). However, their rather folivorous diet (Neville et al. 1988), which may also predict a reduction in the reliance on olfaction, requires controlling for diet before any conclusions regarding the interplay between color vision and olfaction can be drawn. Other confounding factors may be related to non-feeding functions of olfaction. Although some social signals are processed by the independent accessory olfactory system in mammals, the main and accessory olfactory systems show some functional overlap (Baxi et al. 2006). Thus, the main olfactory system is expected to respond to selection pressures from various non-feeding behaviors. Crucially, diet and activity time are somewhat correlated with social structure in primates: many insectivores are also nocturnal and solitary. Hence, it is likely that high olfactory 
capabilities are promoted by one factor and are secondarily employed in other contexts, making it difficult to determine the relative contribution of each factor.

The problem of numerous confounding factors can only be addressed if future studies take a more comparative approach and are designed to control for all relevant variables. For instance, contrasting howler monkeys with muriquis (Brachyteles sp.), the other rather folivorous New World primate who does not share howlers' habitual trichromacy, could help resolve the question whether trichromatic vision is associated with lesser reliance on olfaction. Moreover, perhaps the most efficient approach to control for the relevant factors would be to focus on intraspecific variation and compare conspecific di- and trichromatic New World monkeys or lemurs.

\subsubsection{Comparison to other taxa}

Finally, the processes examined here are not confined to the primate order. Other taxonomic groups such as bats or birds are at least as ecologically diverse and also possess other strong sensory modalities which may have had an effect on olfactory evolution. Comparative anatomical and genetic studies that looked at carnivores (Gittleman 1991), birds (Healy and Guilford 1990; Steiger et al. 2008), bats (Hutcheon et al. 2002) and other mammals (Hayden et al. 2010) are now available. Behavioral studies showed both similar and different trends: long-distance olfactorybased detection is common in procellariiform birds (Nevitt 2000), whereas bats use olfaction in both detection and selection (Thies et al. 1998; Korine and Kalko 2005). Considering the similarities and differences between primates and other taxa would shed additional light on ecological adaptations, proximate mechanisms and constraints and help unravel universal trends in olfactory ecology and evolution.

\section{Acknowledgments}


Olfaction in primate feeding ecology - What next?

We thank John Fleagle, Luca Pozzi and several anonymous reviewers for many helpful comments on previous drafts of this manuscript. We also thank all members of the Behavioral Ecology \& Sociobiology Unit, German Primate Center, for their ideas and remarks in many discussions. 


\section{FRUIT AROMA: ADAPTATION TO PRIMATE SEED DISPERSAL?}

Fruit odor as a ripeness signal for seed-dispersing primates? A case study on four Neotropical plant species

In press

Journal of Chemical Ecology

DOI: 10.1007/s10886-016-0687-x

With:

Eckhard W. Heymann, Behavioral Ecology and Sociobiology Unit, German Primate Center, Kellnerweg 4, 37077 Göttingen, Germany

Stefan Schulz, Institute of Organic Chemistry, Technische Universität Braunschweig, Hagenring 30, 38106 Braunschweig, Germany

Manfred Ayasse, Institute of Evolutionary Ecology and Conservation Genomics, University of UIm, Helmholtzstr. 10-1, containerdorf, 89081 Ulm, Germany 
Fruit aroma: adaptation to primate seed dispersal?

\section{Abstract}

Fleshy fruits contain a myriad of secondary metabolites which may fulfill various non-mutually exclusive ecological functions. Among them are defense against pathogens and herbivores, manipulation of frugivores' gut retention time or controlling the germination process. In addition, it has been suggested that aroma compounds may be used as fruit-selection cues by frugivores and that plants may be under selection to provide a reliable signal for ripeness to seed-dispersal vectors through ripe fruit aroma. A previous project has demonstrated that fruit odor of two Neotropical primate-dispersed plant species can be used by primates to identify ripe fruits. Here, we provide preliminary data supporting the hypothesis that olfactory conspicuousness of ripeness in these two species may be an evolved signal rather than a cue exploited by primates. We analyzed the odors of ripe and unripe fruits of the two species along with odors of two sympatric species whose main dispersal vector is passerine birds. We show that only primate-dispersed species significantly change their odor profiles upon ripening. Thus, odor of bird-dispersed species is not informative regarding their ripeness level and is likely to fulfill other functions. We discuss these data in light of the multiple hypotheses for the presence of fruit secondary metabolites and offer a roadmap for future studies to establish the hypothesis that fruit odor is an evolved signal for ripeness. 
Fruit aroma: adaptation to primate seed dispersal? - Introduction

\subsection{Introduction}

Plants synthesize over 100,000 different secondary metabolites (PSMs) that fulfill a myriad of functions (Knudsen et al. 2006). In vegetative parts, the main function of PSMs is direct or indirect defense against pathogens and herbivors. Flowers and fruits also utilize PSMs for defense. However, in many species they also interact with animal vectors of pollination and seed dispersal and hence their PSM profiles are subjected to multidirectional, sometimes conflicting, selection pressures. While many questions remain unresolved, the biochemistry, ecology and evolution of floral fragrance, i.e. their volatile PSM profiles, have received a great deal of attention over the past decades. In contrast, inquiries into the nature of fruit PSMs, especially volatiles, have lagged behind.

\section{Why do fruits contain secondary metabolites?}

Several hypotheses have been put forward to explain the presence of fruit PSMs. A commonly held null hypothesis is that their presence in fruits derives directly from synthesis of defensive compounds in vegetative parts or unripe fruits and thus requires little further explanation (Eriksson and Ehrlén 1998). However, PSM profiles of ripe fruits are not strictly correlated with those of leaves or unripe fruits (Whitehead and Bowers 2013). This indicates that fruit PSMs may go beyond pleiotropy.

Cipollini and Levey (1997) have suggested several non-mutually exclusive adaptive functions of fruit PSMs. Like in vegetative organs, PSMs can play a defensive role. They can be of general toxicity and thus also deter mutualists (the defense trade-off hypothesis). For example, iridoid glycosides in a hybrid bush honeysuckle defend fruits against pathogens and insects but may deter birds - a major seed disperser (Whitehead and Bowers 2013). Alternatively, PSMs can be toxic to antagonists without deterring legitimate dispersers (directed toxicity). For instance, capsaicinoids in chilli peppers (Capsicum spp.) deter mammalian seed predators without decreasing the fruits' palatability to seed-dispersing birds (Tewksbury and Nabhan 2001). 
Fruit aroma: adaptation to primate seed dispersal? - Introduction

Nonetheless, an a-priori assumption that fruit PSMs play a defensive role is unwarranted. For example, terpenoids are often considered to be defensive compounds. Yet downregulation of limonene synthesis in Citrus fruits renders the fruits less susceptible to microbial pathogens and less attractive to invertebrate antagonists (Rodríguez et al. 2011).

Somewhat ironically, the attraction/repulsion hypothesis postulates that defensive PSMs may be directed against legitimate vertebrate dispersal vectors in order to prevent excessive feeding by a single seed disperser and subsequent deposition of the seeds in a clump. A variation of the attraction/repulsion hypothesis is the protein assimilation hypothesis, according to which PSMs may inhibit protein assimilation by frugivores and thus promote further movement and deposition of the seeds away from the mother tree. These hypotheses have so far received little support.

Ripe fruit PSMs can also manipulate the frugivore's gut retention time in order to maximize the dispersal distance while avoiding excessive damage during digestion. Yet while few studies demonstrated that some fruit PSMs alter frugivores' gut-retention time, none has unequivocally established a connection to an increased fitness benefit (e.g. Wahaj et al. 1998). In addition, PSMs may function in inhibiting seed germination. This ensures that the seeds do not germinate prematurely, and the removal of the pulp by frugivores triggers germination after the dispersal event (Cipollini and Levey 1997). Several studies have reported inhibiting effects of either individual compounds, fruit pulp or pulp extracts. However, the effect is not universal (a compound which inhibits germination in one species does not necessarily have the same effect in others) and it is not always clear whether inhibition has a positive effect on individual plants' fitness (e.g. Wahaj et al. 1998).

\section{PSMs as seed-disperser attractants}

Fleshy fruits are selected to be attractive and PSMs have also been suggested to act as frugivore attractants. The attraction/association hypothesis postulates that PSMs may provide cues regarding fruit ripeness or quality. They can do so through visual cues (pigments such as 
Fruit aroma: adaptation to primate seed dispersal? - Introduction

carotenoids and anthocyanins) or via the chemical senses through aroma compounds. Candidates for such compounds are those whose biosynthesis is directly associated with desired macronutrients (Cipollini and Levey 1997). For example, nitrogen-containing compounds derive from amino acid metabolism (Knudsen et al. 2006) and can therefore provide an honest signal for protein content. In this case it is difficult to distinguish between cues, which provide information but are not necessarily selected to fulfill this function- and signals, which are selected to convey information.

PSMs may also provide act as signals directed at frugivores even without direct biochemical association with the reward. For instance, lipid content in a community of Mediterranean fleshy fruits is positively correlated with fruit color, which in turn promotes consumption by birds (Schaefer et al. 2014). A similar process has been observed in pollination systems (Schiestl 2015). The logic here is that generalist mutualists can learn to prefer plants that provide a signal and "punish" cheaters. Thus, through repeating interactions over evolutionary time-scales, reliable signals may be selected. Aroma compounds which reliably signal a fruit's ripeness and promote ingestion and further foraging on the same plant may even be selected to be present in the pulp rather than the husk. They may be favored in fruits whose thick, leathery or non-permeable husk constrains efficient odor emission

$\underline{\text { Volatile PSMs - fruit odor as a signal for vertebrate dispersal vectors }}$

The hypothesis that fruit odor, its volatile PSMs profile, can be a signal to seed dispersers is not new. Yet in practice it has been rarely addressed in both empirical studies and theoretical considerations. For example, in their thorough book on plant-animal communication, Schaefer and Ruxton (2011) discuss the potential roles of ethylene and ethanol as odor cues, not signals. The strongest evidence that plants employ volatile PSMs to signal ripeness to frugivores comes from a comparison of different fig species (Ficus spp.). The odor of ripe bat-dispersed figs is attractive to them and thus mediate the communication between the plants and their primary seed 
Fruit aroma: adaptation to primate seed dispersal? - Introduction

dispersers (Hodgkison et al. 2013). Bat-dispersed figs also change their odor profile upon ripening, which allows bats to easily detect and identify the ripe fruits; at the same time, birddispersed species do not show a pronounced shift in odor at ripeness, thus indicating that the shift of odor in bat-dispersed figs is not a trait that characterizes all figs but only those which rely on olfactory-guided bats for seed dispersal (Borges et al. 2008). Finally, bat-dispersed fig species tend to emit higher amounts of odor than do bird-dispersed figs (Lomáscolo et al. 2010). Overall, this mirrors the patterns known in pollination ecology: bat-pollinated flowers tend to be odiferous while bird-pollinated flowers are usually scentless. Otherwise, studies addressing this question are practically absent and most data available on ripe fruit odor bouquets come from cultivated species and is therefore less relevant.

In a recent project on the role of primate olfaction in fruit selection, we showed that spider monkeys (Ateles geoffroyi), Neotropical frugivorous primates which are important seed disperses, can readily discriminate between odors of ripe and unripe (husk or pulp) fruits of two primate-dispersed Neotropical species, Couma macrocarpa (Apocynaceae) and Leonia cymosa (Violaceae) (Nevo et al. 2015). This is consistent with the observation that in food acquisition, primates employ their sense of smell mainly for quality assessment of individual fruits (Nevo and Heymann 2015). Primates are important seed dispersers in the tropics and in the Neotropics they constitute a significant part of a more-or-less discrete mammalian dispersal syndrome. Thus, there are many fruiting species that rely on the dispersal services of primates and other similar arboreal mammals and they may be a suitable model system to test the hypothesis that the odors used by primates are not only cues but evolved signals.

Here, we present preliminary data supporting this hypothesis. We compared patterns of odor emission between C. macrocarpa and L. cymosa and two sympatric bird-dispersed species, under the assumption that passerine-dispersed fruits tend to signal through visual rather than olfactory signals. We sampled fruit odors of ripe and unripe, intact and open (husk and pulp odor) fruits of the two primate-dispersed species and of bird-dispersed Maieta guianensis (Melastomataceae) and Psychotria cincta (Rubiaceae). Compound identity and concentration were analyzed using 
Fruit aroma: adaptation to primate seed dispersal? - Methods and materials

gas-chromatography coupled with mass-spectrometry. In each species, we examined whether odor profiles of ripe and unripe fruits are significantly different from one another, either at the intact (husk odor) or open (pulp odor) conditions. The logic is that due to the various functions of fruit PSMs, fruits of all species, ripe and unripe, are expected to emit at least trace amounts of odor. Yet crucially, most non-signaling functions can be achieved by a similar odor bouquet whereas signaling to seed dispersers requires a substantial change in the odor profile to reliably signal ripeness. Thus, it was predicted that if odor has evolved as a signal for vertebrates, a significant shift in the odor profile upon ripening is expected only in species that rely on olfactory-guided primates.

\subsection{Methods and materials}

Fruits from different trees were enclosed in unused oven bags (Toppits, Germany). After $2.5 \mathrm{~h}$ their headspace was collected for 10 min onto a self-produced absorbent Chromatoprobe trap (1.5 mg Tenax-TA, $1.5 \mathrm{mg}$ Carbotrap. Both Supelco, Sigma-Aldrich, Germany). Samples were kept frozen in $-20^{\circ} \mathrm{C}$ and analyzed on a Hewlett Packard 6890 Series gas chromatographic-mass selective detector (GC-MS; Agilent Quadrupol 5972) equipped with a DB-5ms capillary column (30 m long, $250 \mu \mathrm{m}$ in diameter, film thickness: $0.25 \mu \mathrm{m}$, J\&W). Sample size was $N=9$ to $N=15$ fruits per species/condition. Compounds were tentatively identified by comparing their mass spectra and retention indices with published data. Identity of the majority of dominant compounds was confirmed by running synthetic compounds in identical conditions. Data were analyzed for each species separately. Principal component analyses were used to reduce the number of variables in the dataset and eliminate collinearity between them. Then, PCs which explained at least $90 \%$ of the original variance were used in discriminant function analyses and MANOVA tests which examined whether the overall odor bouquet of ripe and unripe fruits differ either in the intact (husk odor) or open (pulp odor) conditions. More details on the study system, sampling and analyses are available in appendix 1. 


\section{3. $\quad$ Results}

Discriminant function analyses and subsequent MANOVA tests revealed clear differences between odor bouquets of ripe and unripe primate-dispersed fruits, but not in the two birddispersed species. In the bird-dispersed $P$. cincta the DFA produced overlapping clusters and the odor of ripe fruits was indistinguishable from that of unripe fruit in both intact and open conditions (Fig. 3.1). The first linear discriminant function (DF1) significantly separated odor samples from the four conditions in the species (ripe/unripe, intact/open) (Wilks' lambda $=0.18$, chi-square $=$ $59.09(15), p<0.001)$ while DF2 did not (Wilks' lambda $=0.69$, chi-square $=12.71(8), p=0.12)$. Consequently, MANOVA tests could not discriminate between the odor profiles of ripe and unripe fruits in either intact $(N=21, F(4,16)=1.69$, adjusted $p=1)$ or open $(N=19, F(4,14)=2.16$, adjusted $p=1$ ) conditions. Similarly, odor profiles of ripe and unripe M. guianensis fruits showed strong overlap in either intact or open conditions. Discrimination was stronger and both DFs were significant $(D F 1$ : Wilks' lambda $=0.2$, chi-square $=58.53(12), p<0.001 ; D F 2:$ Wilks' lambda $=$ 0.68 , chi-square $=14.05(6), p=0.03)$. However, discrimination between odor profiles of ripe and unripe fruits was still low (intact: $N=20, F(4,15)=4.9$, adjusted $p=0.08$. Open: $N=21, F(4,16)$ $=3.31$, adjusted $p=0.3$ ). Thus, in either intact or open conditions, ripe and unripe bird-dispersed species emit odors that are similar and do not provide reliable information regarding their ripeness level.

In contrast, DFAs in primate-dispersed species generated clearly separate clusters of ripe and unripe fruits. In C. macrocarpa the DFA as a whole was significant (DF1: Wilks' lambda $=0.39$, chi-square $=110.58(24), p<0.001 ;$ DF2: Wilks' lambda $=0.46$, chi-square $=26.41(14), p=0.02)$

(Fig. 3.1) and the odor profiles of ripe and unripe fruits were significantly different in both intact ( $N$ $=21, F(8,12)=8.33$, adjusted $p<0.01)$ and open conditions $(N=20, F(8,11)=22.6$, adjusted $p$ $<0.001)$. In L. cymosa (DF1: Wilks' lambda $=0.052$, chi-square $=125.33(21), p<0.001 ;$ DF2: Wilks' lambda $=0.35$, chi-square $=44.95(12), p<0.001)$ the odor profiles of intact ripe and unripe fruits were indistinguishable $(N=25, F(7,17)=2.01$, adjusted $p=0.9)$ but the odor of open ripe 
Fruit aroma: adaptation to primate seed dispersal? - Results

fruits formed a cluster significantly different from the odor of unripe open fruits $(N=24, F(7,16)=$ 12.63 , adjusted $p<0.001)$. Thus, in both species, ripe fruits are characterized by odors that are unique to the ripe phase and are indicative of their ripeness level.

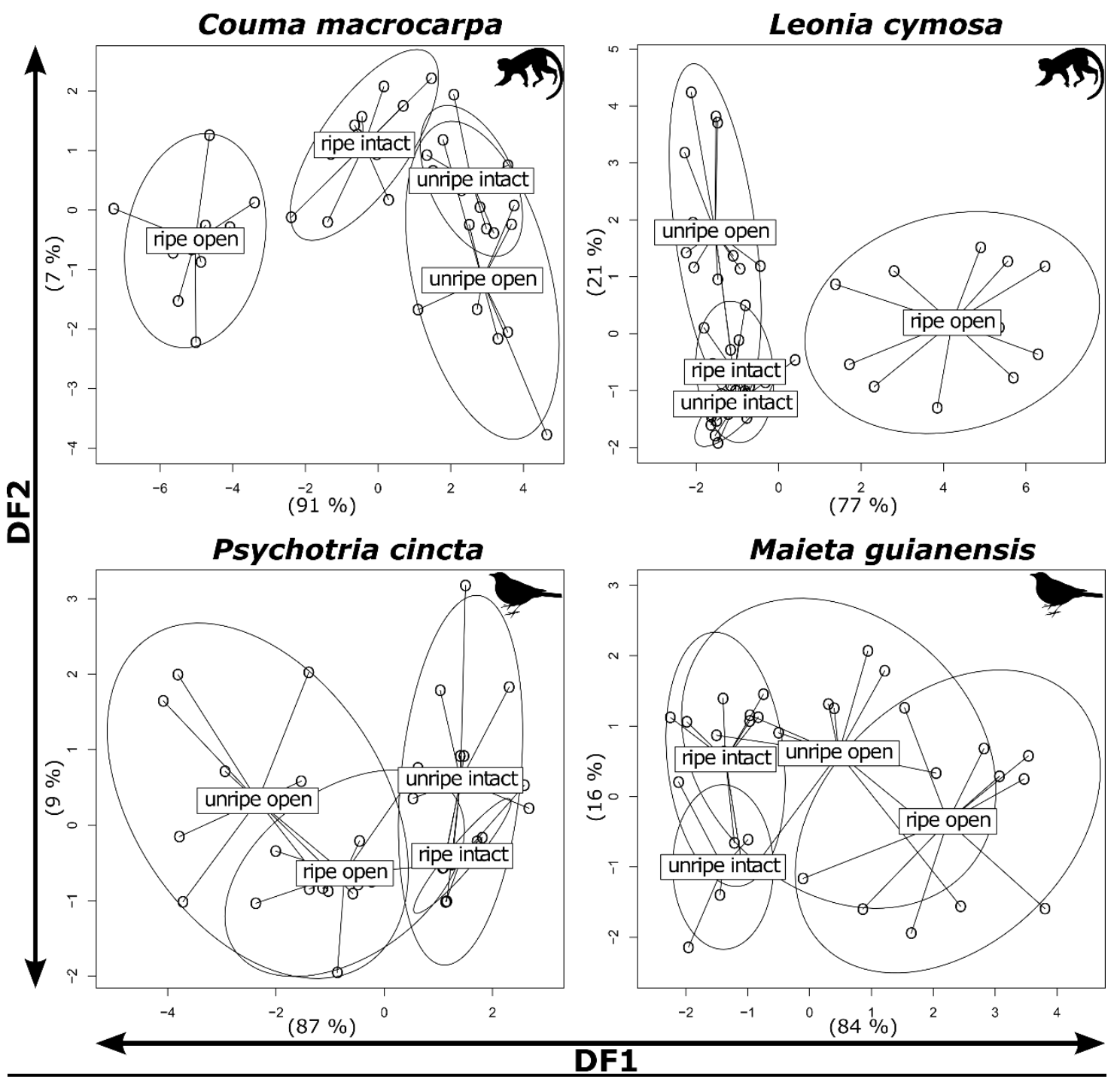

Figure 3.1. Discriminant function analyses to estimate the discrimination potential between odor profiles of ripe and unripe fruits of each species. DFAs were conducted on principal components that accounted for at least $90 \%$ of the original variance (C. macrocarpa: 8 PCs, L. cymosa: 7, P. cincta: 5, M. guianensis: 4). Numbers adjacent to axes are the proportion of between-group variance explained by the respective discriminant function. Clearly distinct odor profile of ripe fruits (intact, open or both) is present only in primate-dispersed species (for test statistics, see $\underline{3.3}$ ) 
Fruit aroma: adaptation to primate seed dispersal? - Discussion

\subsection{Discussion}

Ripe fruits of the two primate-dispersed species emit odors that are significantly different from odors of conspecific unripe fruits (husk, pulp or both), which renders their aromas unique to the ripe phase and reliably convey information to primates, their main dispersal vector (Nevo et al. 2015). In contrast, fruits of the two bird-dispersed species emit odors that are indistinguishable from the odors of unripe fruits. Passerines have elaborated visual capacities and fruits they disperse tend to provide very conspicuous visual cues (Lomáscolo and Schaefer 2010). Thus, it is possible that $M$. guianensis and $P$. cincta have been under selection to focus their signaling efforts on the visual cues. This pattern is similar to bird-pollinated flowers, which often emit only trace amounts of odors and provide conspicuous visual signals. A possible function of the volatile compounds identified is defense, since in contrast to primate-dispersed species, in order to be available to small understory passerines these and similar fruits are soft and only lightly protected by a protective husk. Thus, they are more susceptible to pathogens and herbivores than $C$. macrocarpa and L. cymosa, which are protected by a thick husk. Other alternative explanations may also explain the presence of these volatiles in the two bird-dispersed species.

Each of the primate-dispersed species is phylogenetically closer to one of the bird-dispersed species than they are to one another (see appendix 1). Taken together, these results indicate that the olfactory conspicuousness - the substantial shift in odor profile upon ripeness - of $C$. macrocarpa and L. cymosa may not be an inevitable characteristic of fleshy-fruit maturation which can be used by primates as a cue for fruit selection, but possibly an evolved signal whose function is to convey information to seed-dispersing primates. At the same time, these conclusions are based on only four species and it is hence impossible to extrapolate from them to the entire system. Thus, while they indicate that signaling ripeness to seed-dispersing mammals may be an evolved function of fruit odor, this hypothesis remains to be established. 
Fruit aroma: adaptation to primate seed dispersal? - Discussion

\subsubsection{How to continue? A roadmap for inferring whether fruit odor is an evolved signal for} ripeness

Inferring adaptation is never a straightforward task, especially in multidimensional traits such as fruit odor and when various, non-mutually exclusive, alternative explanations are applicable. We therefore suggest three approaches which together can establish the hypothesis that signaling to vertebrates is one of the functions PSMs fulfill in some taxa: the ecological, comparative and integrative approaches.

\section{Ecological approach}

Before asking whether fruit odor is an evolved signal directed at vertebrates, it must be established that it is at the very least used as a cue, i.e. that seed-dispersal vectors indeed rely on in the process of food selection. This can be done both in observational studies that quantify sniffing behavior of frugivores in the wild or in controlled experiments that annihilate other cues and ask whether the vertebrate can identify ripe fruits in the absence of cues from other sensory modalities. A second prediction that should be confirmed is that these olfactory cues are not redundant in the more natural scenario, when cues from other sensory modalities are available. Here, an effective experimental design could present vertebrates with choice tests between items that provide visual cues, olfactory cues or a combination of both. The expectation is that the presence of odor provides non-redundant information regarding fruit maturity and hence the combination of olfactory and visual cues should be preferred over visual cues alone. Finally, the hypothesis that some components of fruit odor have evolved, or are maintained, by selection by vertebrate frugivores also requires that these vertebrates would show consistent preference for fruits with a more pronounced signal.

\section{Comparative approach}


Fruit aroma: adaptation to primate seed dispersal? - Discussion

A second phase in establishing that that fruit odor is an evolved signal for ripeness is to demonstrate its absence in plant lineages that rely on dispersal services by animals which are less likely use olfaction in fruit selection. Given the alternative adaptive and non-adaptive explanations for the presence of fruit PSMs, all fruits are likely to emit some VOCs (volatile organic compounds). Thus, the prediction in a comparative study would not be that fruits whose primary seed dispersers are not olfactory-guided do not have an odor at all, but that their odors are substantially less informative than those of fruits that depend on olfactory-guided vertebrates. Hence, the main prediction should be that all other things being equal, the former emit odor bouquets that are not significantly different between ripe and unripe fruits while the latter show a pronounced shift in the VOC profile upon ripening. While the data presented above provide preliminary support that this is the case in four Neotropical species, confirmation of this hypothesis would require a much larger dataset that would allow quantitative phylogenetic-controlled analyses.

$\underline{\text { Integrative approach }}$

Finally, another approach that would help establishing that the odors vertebrates use in fruit selection are signals rather than cues is an examination, and exclusion, of alternative explanations. Once it is established that fruit odor mediates the communication between seed dispersers and plants (i.e. that it is a cue), exclusion of alternative hypotheses would increase the probability that a major selection pressure for the presence of ripe fruits VOCs is selection by frugivores. For example, biochemical pleiotropy can be excluded if odor profiles of ripe fruits are significantly different, quantitatively or qualitatively, from VOC profiles of other plant organs and unripe fruits. Adaptive functions can be tested using various designs. Antibiotic properties of odor components can be estimated using classical antibiotic tests or through genetic manipulation of odor profiles and comparison of the susceptibility of wild-type and genetically-modified fruits to various antagonists. Other functions can be tested using both correlational studies of natural populations or controlled experiments. 
Fruit aroma: adaptation to primate seed dispersal? - Conclusions

This approach, however, suffers from several shortcomings. First, fruit volatiles may fulfill many different functions, some not yet conceived and eliminating all might prove impossible. Second, in many cases it is impossible to fully exclude a function. For example, a study can show that certain VOCs in ripe fruits are not effective defenders against pathogens which are known to attack the fruit. Yet it could still be that these substances are highly effective against another pathogen which is not even identified as an antagonist of the plant species due to its effective defense mechanisms. In this case, a highly effective defensive compound may actually be considered to have no defensive properties. Third, PSMs may act in concert with other PSMs or require specific conditions which are difficult to replicate in controlled experiments. Finally, PSMs could fulfill various non-mutually exclusive functions, or their contemporary main function could be a secondary adaptation. For example, floral odorants which convey information to pollinating invertebrates are believed to have originally evolved as defensive barriers. Thus, filtering out the relative role of each function on the presence of a certain compound or compound group is neither sufficient nor straightforward.

\subsection{Conclusions}

Fruit PSMs have been suggested to fulfill various, non-mutually-exclusive functions. An attractive, yet under-investigated, hypothesis is that their role is to signal ripeness to seed-dispersing vertebrates. The data presented here provide preliminary support for this hypothesis. Yet to establish this hypothesis many factors ought to be controlled. We suggest three approaches which, together, may allow to disentangle this complex question and we are in hope that future studies will take this path to provide more established answers to the question whether fleshy fruits whose main dispersal vectors are olfactory-dependent vertebrates have evolved to communicate with them via olfactory signals. 
Fruit aroma: adaptation to primate seed dispersal? - Conclusions

\section{Acknowledgments}

We thank John Romeo, André Kessler, and several anonymous reviewers for many helpful comments on previous drafts of this manuscript. ON was funded by the Minerva Fellowship during his work on the data presented. Project expenses were covered by the German Science Foundation (DFG), grants HE 1870/19-01 and AY 12/7-1. Samples were collected under research permit n. 0329-2012-AG-DGFFS-DGEFFS issued by the Peruvian Ministry of Agriculture. We further thank Shereen Petersen for proofreading the manuscript 


\section{USE OF FRUIT ODOR FOR FRUIT CHOICE IN BLACK-HANDED SPIDER MONKEYS}

\section{Chemical recognition of fruit ripeness in spider monkeys, Ateles geoffroyi}

\section{5}

\section{Scientific Reports 5: 14895}

DOI: 10.1038/srep14895

With:

Rosa Orts Garri (shared first authorship), IFM Biology, Linköping University, 58183 Linköping, Sweden

Manfred Ayasse, Institute of Evolutionary Ecology and Conservation Genomics, University of Ulm, Helmholtzstr. 10-1, containerdorf, 89081 Ulm, Germany

Laura Teresa Hernandez Salazar, Institute of Neuroethology, University of Veracruzana, Calle y No. Dr. Luis Castelazo s/n, Col. Industrial Animas, C.P. 91190, Ciudad, Xalapa, Ver., México

Eckhard W. Heymann, Behavioral Ecology and Sociobiology Unit, German Primate Center, Kellnerweg 4, 37077 Göttingen, Germany

Stefan Schulz, Institute of Organic Chemistry, Technische Universität Braunschweig, Hagenring 30, 38106 Braunschweig, Germany

Matthias Laska, IFM Biology, Linköping University, 58183 Linköping, Sweden $\underline{\text { Abstract }}$ 
Primates are now known to possess well-developed olfactory sensitivity and discrimination capacities that can play a substantial role in many aspects of their interaction with conspecifics and the environment. Several studies have demonstrated that olfactory cues may be useful in fruit selection. Here, using a conditioning paradigm, we show that captive spider monkeys (Ateles geoffroyi) display high olfactory discrimination performance between synthetic odor mixtures mimicking ripe and unripe fruits of two wild, primate-consumed, Neotropical plant species. Further, we show that spider monkeys are able to discriminate the odor of ripe fruits from odors that simulate unripe fruits that become increasingly similar to that of ripe ones. These results suggest that the ability of spider monkeys to identify ripe fruits may not depend on the presence of any individual compound that mark fruit ripeness. Further, the results demonstrate that spider monkeys are able to identify ripe fruits even when the odor signal is accompanied by a substantial degree of noise. 


\subsection{Introduction}

Primates have traditionally been considered as primarily visually-oriented animals with a poorly developed sense of smell (Le Gros Clark 1971; Fobes and King 1982). This view was mainly, if not exclusively, based on an interpretation of neuroanatomical and - more recently - genetic findings and not on behavioral or physiological evidence (Laska et al. 2005a). Meanwhile, an increasing number of studies now suggest that olfaction may play a significant role in regulating a wide variety of primate behaviors (Michael et al. 1976; Kappel et al. 2011) and that the olfactory sensitivity and discrimination capacities of both human and nonhuman primates are not generally inferior to that of nonprimate species believed to have a keen sense of smell (Laska et al. 2000).

A high olfactory sensitivity and well-developed olfactory discrimination capabilities have been reported in several primate species of different lineages (Hübener and Laska 1998; Laska et al. 2000, 2005a,Laska and Seibt 2002b). These olfactory capabilities have been shown to be particularly tuned to detection, discrimination and identification of compounds common in fruits (Ueno 1994,Laska and Seibt 2002a,b; Hernandez Salazar et al. 2003,Laska et al. 2006a). Additionally, behavioral studies from captivity and the wild demonstrated that non-human primates can rely on olfactory cues in fruit selection tasks (Nevo and Heymann 2015; Pablo-Rodríguez et al. 2015).

While psychophysical studies quantified the olfactory sensitivity for and discrimination performance of primates with compounds that are potentially relevant for feeding on fruits (Laska and Seibt 2002b,Laska et al. 2006a), they all used monomolecular stimuli. Yet fruit odor is composed of complex mixtures of odorants (Hodgkison et al. 2007, 2013) and in natural fruitselection primates need to identify complex odor signatures of ripe fruits and be able to discriminate them from odor profiles of unripe fruits. Thus, although these studies showed a potential to rely on olfactory cues in frugivory, they could not demonstrate whether and how high olfactory sensitivity and discrimination capacity translate into success in food acquisition tasks mimicking a real-life situation. On the other hand, most behavioral studies that employed more ecologically-realistic approaches used chemically undefined stimuli (Bicca-Marques and Garber 
Use of fruit odor for fruit choice in black-handed spider monkeys - Introduction

2004; Hiramatsu et al. 2009; Melin et al. 2009) or chemically known stimuli of domesticated fruit species (Rushmore et al. 2012) whose odor profiles may have been enhanced through artificial selection and are therefore not representative of the challenges primates face when selecting fruits in the wild. So, the connection between primate olfactory physiology and feeding ecology is not yet fully established. First, the ability to discriminate between complex odor mixtures that mimic odors of wild ripe and unripe fruits has not been experimentally demonstrated. Second, it is unknown whether sensitivity to, or discrimination of, any particular compound or compound family disproportionally increases their capability to identify ripe fruits.

A recent study (Nevo et al., unpublished data) provided detailed analyses of the chemical profiles of the fruit odors of two Neotropical plant species whose seeds are dispersed primarily by primates. By comparison to patterns of odor emission in fruits whose main seed-dispersal vectors are birds, which are demonstrated to be less olfactory-, and more visually, dependent, it was hypothesized that odors of ripe fruits consumed by primates are not merely a cue that primates can potentially exploit, but an evolved signal whose function is to facilitate the communication between seed-dispersing primates and plants. More specifically, it was suggested that since primates and other, extant or extinct, frugivores tend to use olfactory cues in fruit selection (Nevo and Heymann 2015), fruits that rely on their seed-dispersal services have evolved to emit an odor which is unique to the ripe phase, i.e. significantly different from the odor of unripe fruits of the same species. This was hypothesized to increase the ability of primates and other frugivores to identify ripe fruits when selecting between ripe and unripe fruits in a feeding tree and hence their foraging efficiency. In turn, this should increase the overall attractiveness of a fruit and allow the plant to outcompete con- and heterospecifics in attracting dispersal vectors. However, without bioassays that test the ability of primates to detect the odors and discriminate them from the odors of unripe fruits, this claim remains tentative.

The current study attempts to build upon these chemical analyses of odors emitted by primateconsumed fruits and takes a step forward in connecting primate olfactory physiology and feeding ecology. Nevo et al. provide chemical characterizations of the odors of ripe and unripe fruits of 
Use of fruit odor for fruit choice in black-handed spider monkeys - Introduction

two Neotropical plant species, Couma macrocarpa (Apocynaceae) and Leonia cymosa (Violaceae). Both plant species provide indehiscent fruits (i.e. whose peel does not open upon maturation) with a soft, leathery peel that acquires a yellow color when ripening. Analyses of the chemical profiles revealed that in one species, C. macrocarpa, peel odor (i.e. odor of the intact fruits) profiles of ripe and unripe fruits are strong, rich and distinct (i.e. different between ripe and unripe fruits). In contrast, intact ripe and unripe L. cymosa fruits bear weak and similar odors, whereas the pulp (open fruits) is very odorous and its composition differs significantly between ripe and unripe fruits. Thus, it was predicted that in C. macrocarpa the odor of intact fruits would be sufficient to inform primates that an individual fruit is ripe while in L. cymosa primates must first manipulate the fruits and expose the pulp, and only in this phase receive the signal that the fruit is ripe.

The goal of the current study was to assess whether the odor profiles of fruits of these two species indeed have the potential to mediate the interaction between plants and primates by signaling ripeness at the fruit selection phase, and to examine whether this ability depends on few compounds or compound classes that characterize the odors of ripe fruits. Using five captive black-handed spider monkeys (Ateles geoffroyi) as a model system, we addressed the following questions:

1. Can spider monkeys discriminate between odor profiles of ripe and unripe fruits (C. macrocarpa: peel odor, L. cymosa: peel and pulp odor)?

2. Does the ability to discriminate between odor profiles of ripe and unripe fruits of a certain plant species depend on one or a few compounds or compound classes that mark ripe fruits? Are odor profiles of unripe fruits that are more similar to the odor of ripe fruits with regards to these compounds more difficult to discriminate from the odor of ripe fruits?

To address these questions, we employed an olfactory conditioning paradigm (Laska et al. 2003). We used synthetic odor mixtures that mimicked the odors of ripe and unripe fruits (peel odor in $C$. macrocarpa and both peel and pulp odors in L. cymosa). We first trained the monkeys to identify 
the odor of ripe fruits and associate it with a food reward. Then, to address the first question, we tested the ability of spider monkeys to discriminate between the odors of ripe and unripe fruits of each species in a given condition (intact or open) using the full odor mixtures (i.e. those mimicking the natural odors of ripe and unripe fruits, respectively, as closely as possible). To address the second question, we employed a series of similar experiments in which we tested the ability of spider monkeys to discriminate between the odor of ripe fruits and the odor of "partially ripe" fruits. Partially ripe fruits were odor mixtures similar to the odor of unripe fruits which were manipulated to resemble the odor of ripe fruits with regards to one or more compounds (i.e. the concentration of compounds was manipulated so that one or more compounds in the odor of unripe fruits were matched to their respective concentration in the odor of ripe fruits). Thus, in each of these experiments, the odor of unripe fruits became more similar to the odor of ripe fruits with regards to one or more compound while all the other odorants in the mixture remained at concentrations appropriate for unripe fruits. The question was whether this would decrease the monkeys' ability to discriminate between the odors.

\section{2. $\quad \underline{\text { Results }}$}

\subsubsection{Couma macrocarpa - peel odor (intact fruits)}

Figure 4.1 shows the performance of the five spider monkeys in the training phase (Fig. 4.1, odor pair 1) and in discriminating between the odor of intact ripe and unripe fruits of Couma macrocarpa (Q1, odor pair 2) and odors mimicking different degrees of ripeness of this fruit (Q2, odor pairs 313). With all 13 odor pairs, either all five animals ( 6 cases), or at least the majority of animals (7 cases) scored $\geq 70.0 \%$ correct decisions (corresponding to $p<0.05$ in a binomial test; see methods) and therefore succeeded in discriminating between the stimuli above chance level. With 11 of the 13 odor pairs, the majority of animals even scored $\geq 76.7 \%$ correct decisions (corresponding to $p<0.01$ ). Thus, the spider monkeys were clearly able to distinguish between odors of intact ripe and unripe fruits (Q1) and between ripe fruits and partially ripe fruits (Q2). 
Discrimination performance did not differ between treatments (Friedman's test: $n=5(11), x 2=$ 14.59, $p=0.2$ ), implying that none of the odor mixtures, including partially ripe odor mixtures that were more similar to the fully-ripe odor, were more difficult to discriminate compared to other odor mixtures.

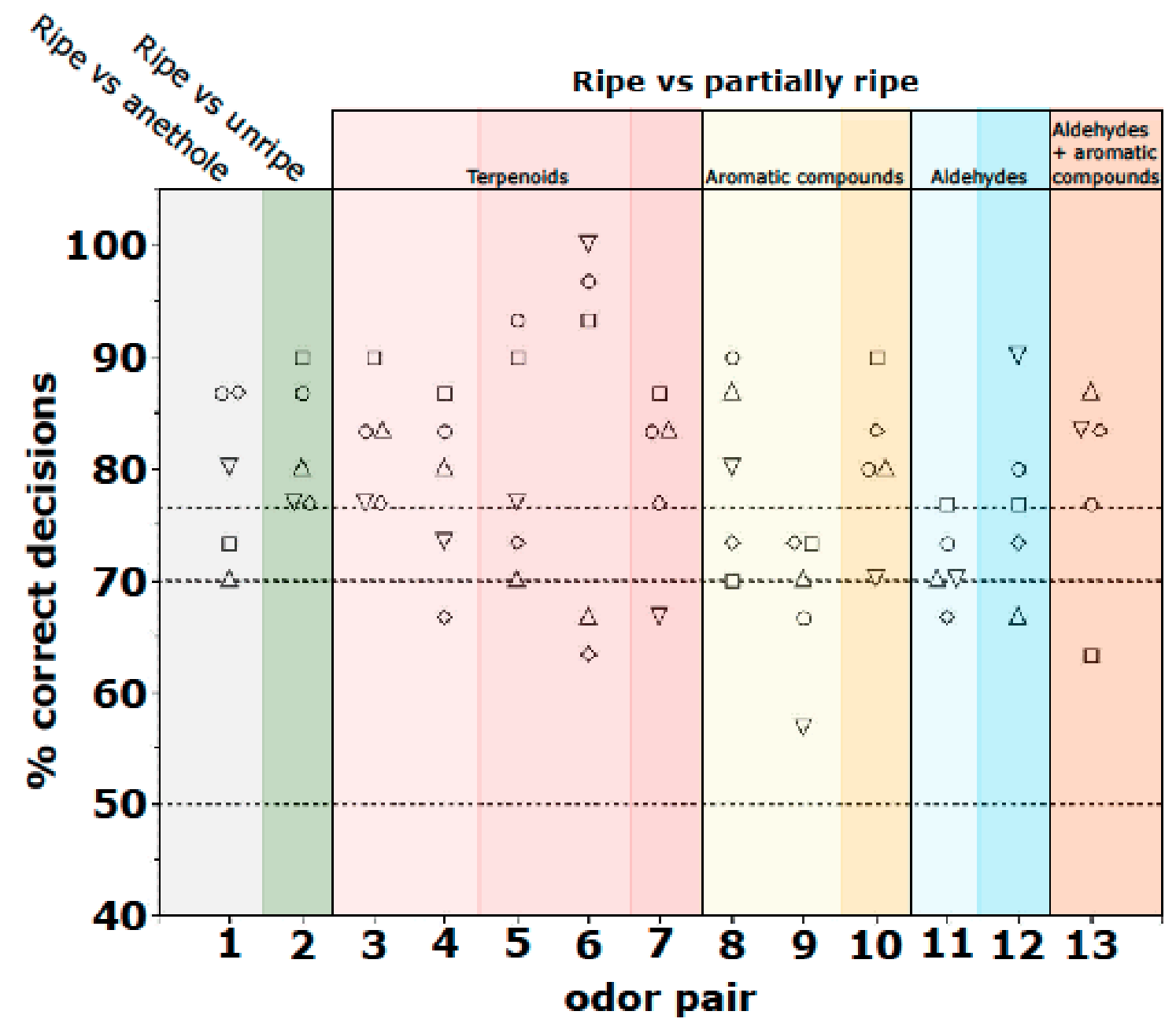

Figure 4.1.Performance of five spider monkeys in discriminating between the odor of ripe intact fruits of Couma macrocarpa and odor mixtures mimicking different degrees of ripeness of this fruit. Each data point represents the percentage of correct decisions per odor pair and animal. Horizontal lines indicate chance level at $50 \%$, and criterion levels at $70 \%$ (corresponding to $p<0.05$ in a binomial test; see methods) and at $76.7 \%$ (corresponding to $p<0.01$ ). The numbers and composition of odor pairs are given in Table 4.1. Anethole (odor pair 1 ) served as a monomolecular training stimulus. Ripe vs unripe (odor pair 2) corresponds to question 1 from the introduction. Odor pairs 3-13 correspond to question 2. Colors in odor pairs 3-13 mark different odorant categories and darker shades within them (left to right) indicate increasingly ripe odor mixtures within these categories. 


\subsubsection{Leonia cymosa - peel odor (intact fruits)}

Discrimination performance between odor profiles of ripe and unripe intact Leonia cymosa odors was overall very low. Mean success rates of 4 out of 5 spider monkeys was lower than $70 \%$ (mean: 58\%) and thus not different from chance whereas one individual achieved $80 \%$ success. Success rates were equally low in the training phase (ripe fruits vs anethole) (mean: $59 \%$ ). So, as a group, the monkeys showed difficulties in identifying the odor of ripe intact $L$. cymosa fruits and as a result could not recognize ripe fruits based on their odor in the intact condition. To exclude the possibility that the inability to discriminate the odor of ripe intact L. cymosa fruits from anethole or from the odor of intact unripe fruits derives from the inability to detect the odor, we tested the discrimination capacity from water. Success rates of all five individuals were equal to, or higher than, $76.7 \%$ (mean: $80 \%$ ).

\subsubsection{Leonia cymosa - pulp odor (open fruits)}

Figure 4.2 shows the performance of the five spider monkeys in the training phase (Fig. 4.2, odor pair 1) and in discriminating between the odor of open ripe and unripe fruits of Leonia cymosa (Q1, odor pair 2) and odors mimicking different degrees of ripeness of this fruit (Q2, odor pairs 310). With all 10 odor pairs, either all five animals, or at least the majority of animals succeeded in discriminating between the stimuli above chance level $(p<0.05)$. With 8 of the 10 odor pairs, all five animals even scored $\geq 76.7 \%$ correct decisions (corresponding to $p<0.01$ ). Thus, the monkeys readily discriminated between odors of open ripe and unripe fruits and between the odor of ripe fruits and partially ripe fruits.

Differences in discrimination performance between treatments approached significance (Friedman's test: $n=5(8), x^{2}=14.73, p=0.065$ ) but subsequent post-hoc analyses (pair-wise Wilcoxon Signed-Rank tests followed by the Bonferroni correction for multiple testing) revealed 
that discrimination performance in all tasks was statistically indistinguishable (all pairwise comparisons: adjusted $p=1$ ). Thus, similarly to intact $C$. macrocarpa fruits, none of the odor mixtures, including partially ripe odor mixtures that were more similar to the fully-ripe odor, were more difficult to discriminate compared to other odor mixtures.

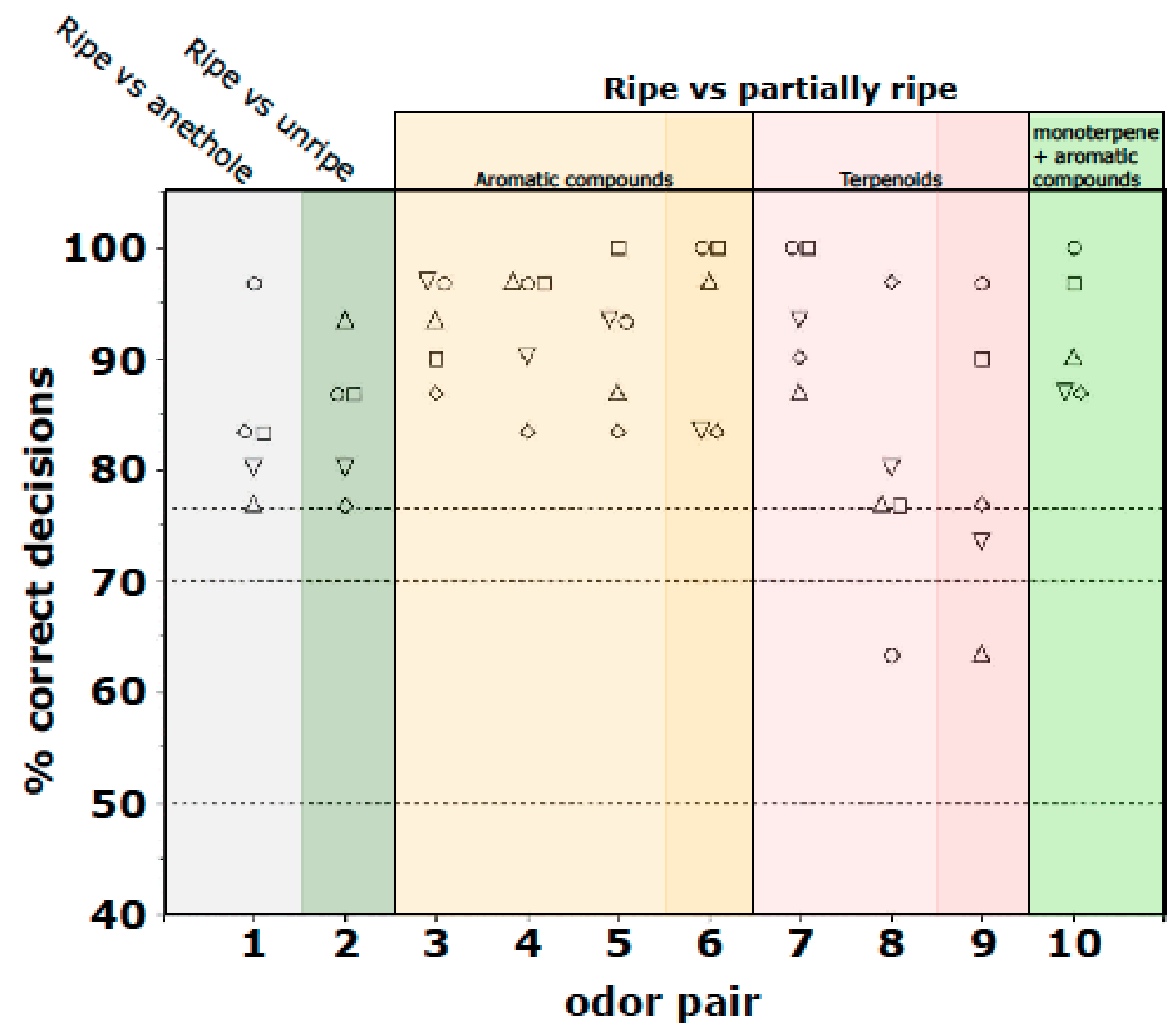

Figure 4.2 Performance of five spider monkeys in discriminating between the odor of ripe open fruits of Leonia cymosa and odor mixtures mimicking different degrees of ripeness of this fruit. Each data point represents the percentage of correct decisions per odor pair and animal. Horizontal lines indicate chance level at $50 \%$, and criterion levels at $70 \%$ (corresponding to $p<0.05$ in a binomial test; see methods) and at $76.7 \%$ (corresponding to $p<0.01$ ). The numbers and composition of odor pairs are given in Table 4.3. Anethole (odor pair 1 ) served as a monomolecular training stimulus. Ripe vs unripe (odor pair 2) corresponds to question 1 from the introduction. Odor pairs 3-10 correspond to question 2. Colors in odor pairs 3-10 mark different odorant categories and darker shades within them (left to right) indicate increasingly ripe odor mixtures within these categories. 


\subsection{Discussion}

The first question we addressed was whether spider monkeys can discriminate between the odors of ripe and unripe fruits. The results were positive in both plant species: in $C$. macrocarpa the animals successfully discriminated between the odors of intact (peel odor) ripe and unripe fruits (Fig. 4.1, odor pair 2); in L. cymosa they failed to do so, but could readily discriminate between the odors of open ripe and unripe fruits (pulp odor) (Fig. 4.2, odor pair 2). These results suggest that spider monkeys can rely on fruit odor for identification of ripe C. macrocarpa and L. cymosa fruits in the wild: during the food-selection process individuals sample the odors of ripe and unripe fruits. In an unordered series of visual, olfactory and tactile examination (Dominy et al. 2006), they are exposed to the odor of both the intact (peel odor) or open (pulp odor) fruit. As a result, they learn to associate the odors of ripe fruits with a reward, in a process that is similar to the conditioning paradigm employed here. Thus, over time, the ability to discriminate between odors of ripe and unripe fruits of these species is likely to translate into the ability to assess the fruits' ripeness based solely on their odor.

These results are in line with the hypothesis that fruit odor in C. macrocarpa and L. cymosa is an evolved signal to seed-dispersing primates and/or other contemporary or extinct frugivores. Nevo et al. showed that the emission of a unique odor at ripeness, either of the intact or open fruits, characterizes plants whose seeds are dispersed by primates and not by birds. Our results confirm that spider monkeys have the ability to discriminate between the odors of ripe and unripe fruits and thus the potential to use fruit odor to identify ripe fruits. While alternative explanations cannot at this point be ruled out, these results indicate that selection by monkeys and other frugivores may have driven an evolution of unique odor at ripeness in fruit species whose seeds they disperse.

The second question we addressed was whether the ability of spider monkeys to discriminate between odors of ripe and unripe fruits depends on one or perhaps a few odorants or odorant classes which may be indicative of ripeness. Using an odor mixture that did not mimic a natural stimulus, a previous study has found that discrimination performance in squirrel monkeys (Saimiri 
Use of fruit odor for fruit choice in black-handed spider monkeys - Discussion

sciureus) decreases when odor mixtures become increasingly similar and that some odorants disproportionally contribute to their ability to do so (Laska and Hudson 1993). Here, using stimuli that mimicked natural fruit odors, only minor differences in the performance of the spider monkeys between the different odor pairs was observed and none was statistically significant. For example, in intact C. macrocarpa, adding methyl salicylate (Fig. 4.1, odor pair 9) to the odor of unripe fruits yielded a slight, but statistically insignificant, decrease in the spider monkeys' discrimination performance. However, when adding all relevant aromatic compounds (Fig. 4.1, odor pair 10: methyl salicylate, ethyl salicylate, p-cymene), which was expected to yield an odor mixture that resembles the odor of ripe fruits even more, the spider monkeys' discrimination performance was slightly higher and statistically indistinguishable from their ability to discriminate between the full ripe and unripe odors (Fig. 4.1, odor pair 2). Thus, we interpret all deviations from the baseline discrimination level (ripe vs. full unripe, odor pair 2) as statistical noise and are left to conclude that none of the manipulations of unripe odors caused any systematic decrease in the ability of the spider monkeys to discriminate between odors of ripe and unripe fruits. In summary, even as the odors of ripe and partially ripe fruits became increasingly similar, spider monkeys still readily discriminated between them and identified the full ripe odor mixture.

These results exemplify the acute sense of smell in spider monkeys and suggest that the ability to identify ripe fruits does not depend on any single compound. Further, these results show that even when the odors of ripe and unripe fruits become increasingly similar, the monkeys still readily discriminate between them and can use olfactory cues to determine whether a fruit is ripe or not. Finally, the results show that the monkeys quickly learn to successfully solve novel olfactory tasks (discrimination between a known rewarding and a variety of novel non-rewarding odors). This ability is beneficial because natural fruit odors are not uniform: individual fruits may develop under different conditions and therefore unripe fruits may emit some compounds in concentrations similar to the ripe fruits, and vice versa (Hodgkison et al. 2007). As a result, the ability to recognize the odor of ripe fruits against different combinations of partially ripe odor should allow spider monkeys to select fruits of an optimal degree of ripeness in a natural environment, in which signals are often accompanied with some degree of noise. 
Use of fruit odor for fruit choice in black-handed spider monkeys - Discussion

Thus, odor profiles of ripe C. macrocarpa and L. cymosa, which are composed of a plethora of different odorants, show a complexity that allows them to remain unique, and hence identifiable by spider monkeys and probably other primates and non-primate frugivores as well, even when the concentration of some compounds substantially deviates from the mean typical for a given ripeness level. This increases the signal's specificity and ensures the reliability of communication despite inevitable noise with regards to the concentration of some compounds, and may therefore be an adapted feature of fruit odor in the two plant species. On the other hand, it should be considered that the biosynthetic machinery used for production of plant secondary metabolites is non-specific, and therefore volatile plant secondary metabolites are always produced in complex mixtures (Fischbach and Clardy 2007). Therefore, it could be that if fruits are under selection to emit an odor that signals their ripeness, the only way to achieve that is through complex mixtures of volatiles. In this case, odor complexity of ripe C. macrocarpa and L. cymosa is an inevitable byproduct of signaling via the olfactory trajectory and it is possible that if a more compoundspecific biosynthetic pathway for synthesis of volatile secondary metabolites were available, a simpler odor mixture could function equally well in conveying information to seed-dispersal vectors.

Our results further highlight that the sense of smell of a species cannot be summed up simply as "good" or "bad". As fruit specialists that feed on fruits of many different plant species (di Fiore et al. 2008), spider monkeys would benefit from the ability to learn olfactory discrimination tasks and maintaining high discrimination ability between complex mixtures even when signals include a substantial amount of noise. Other, more specialist species, may possess olfactory systems that serve them well in their respective ecological niche but which do not require to maintain such high discrimination capacity in diverse, noisy, conditions. So, their olfactory systems may be useful and good - but entail different capacities.

In conclusion, our study provides the first attempt to examine how primate olfactory discrimination capacity translates into success in ecologically realistic fruit-selection tasks. It confirms that spider monkeys achieve high discrimination performance between odor profiles of ripe and unripe fruits 
Use of fruit odor for fruit choice in black-handed spider monkeys - Methods

of two wild plant species, and therefore identify ripe fruits based on their volatile profiles. Further, our results show that the ability of spider monkeys to discriminate between the odors of ripe and unripe fruits does not depend on single compounds or compound classes. This unique odor signature, which retains information regarding fruit ripeness even when some noise is introduced, should enhance the overall attractiveness of the fruits to frugivores and therefore contribute to facilitating the mutually-beneficial interaction between plants and seed-dispersing primates and possibly other seed-dispersal vectors.

\subsection{Methods}

\subsubsection{Animals}

Testing was carried out using four adult female and one adult male black-handed spider monkeys (Ateles geoffroyi). The male was 8 years old, and the females were 9, 10, 11, and 15 years old, respectively, at the start of the study. The spider monkeys were kept in outdoor enclosures at the UMA Hilda O'Farrill (environmental management unit), maintained by the Universidad Veracruzana near Catemaco, Veracruz, Mexico, and were thus exposed to natural environmental conditions concerning ambient temperature, relative humidity, and light. All spider monkeys had served as subjects in previous olfactory experiments and were familiar with the basic test procedure (Løtvedt et al. 2012; Wallén et al. 2012; Sarrafchi et al. 2013). Maintenance of the animals has been described in detail elsewhere (Hernandez Salazar et al. 2003). As they were all captive born, it is unlikely that they had been familiar with fruits of Couma macrocarpa and Leonia cymosa prior to the current experiments.

The experiments reported here comply with the Guide for the Care and Use of Laboratory Animals (National Institutes of Health Publication no. 86-23, revised 1985) and also with current Swedish, German, and Mexican laws. They were performed according to a protocol approved by the ethical board of the Federal Government of Mexico's Secretariat of Environment and Natural Resources (SEMARNAT; Official permits no. 09/GS-2132/05/10). 


\subsection{2. $\underline{\text { Odorants }}$}

Odor stimuli were synthetic odor mixtures mimicking the odors of ripe and unripe fruits of Couma macrocarpa (intact) and Leonia cymosa (intact and open) as well as of intermediate degrees of ripeness of both fruits. For this, we prepared mixtures mimicking the odor of partially-ripe fruits in which the concentration of one or more compounds as present in the unripe fruit was manipulated to match the concentration in the odor of the respective ripe fruit.

We used commercially available odorants (Tab. 9.1) dissolved in near-odorless diethyl phthalate (99\%, Sigma Aldrich, Germany). Although not all odor chemicals identified in the natural fruits (Nevo et al.) were available, we obtained most of the major components. This allowed reconstructing of a substantial proportion of the natural odors (C. macrocarpa - ripe intact: $84 \%$, unripe intact: $84 \%$; L. cymosa - ripe intact: $69 \%$, unripe intact: $77 \%$, ripe open: $87 \%$, unripe open: $68 \%)$.

After mixture preparation we sampled their headspaces to verify that their odors resembled the odors of natural fruits. Sampling was conducted according to a protocol identical to the one used for analysis of natural fruit odor in Nevo et al. $1 \mathrm{ml}$ of mixture was placed in an open $2 \mathrm{ml}$ Eppendorf tube and placed inside a sealed chamber made from an unused inert baking bag (Toppits, Germany) for $2.5 \mathrm{~h}$. The accumulated headspace was then collected for $10 \mathrm{~min}$ in a constant airflow if $330 \mathrm{ml} / \mathrm{min}$ onto a self-made absorbent trap containing $1.5 \mathrm{mg}$ of Tenax-TA and $1.5 \mathrm{mg}$ Carbotrap (both Supelco, Sigma-Aldrich, Germany). Absorbent traps were loaded at the tip of a cleaned Teflon tube which was the only opening in the system. Absorbent traps were loaded immediately afterwards to a Hewlett Packard HP 6890 Series gas chromatographic-mass selective detector (GC-MS; Agilent Quadrupol 5972) equipped with a DB-5ms capillary column (30 m long, $250 \mu \mathrm{m}$ in diameter, film thickness: $0.25 \mu \mathrm{m}, \mathrm{J \& W}$ ) and analyzed in conditions identical to those described in Nevo et al. We then adjusted the concentrations of the odorants in accordance with the results, until the headspace was similar to the odor of natural fruits in both 
composition and intensity. To confirm that our synthetic mixtures sufficiently resembled the natural odors, we ran a principal component analysis (PCA) followed by a discriminant function analyses on the natural odor of ripe and unripe fruits of both species (data and analysis methods from Nevo et al.) and then verified that the synthetic mixtures scored on the DFAs similarly to the natural odors. All samples scored within the range of the natural odors on the discriminant functions and can thus be considered to be reasonable representatives of natural odors (Fig. 9.1, $\underline{\text { 9.2). }}$ ).

This procedure led to 6 basic "recipes" mimicking the full odors of ripe and unripe fruits of $C$. macrocarpa (intact only) and L. cymosa (intact and open) (Tab. 9.2, $\underline{9.3}$ ), which we also used to generate the partially ripe odors. Due to time and budget constraints, we did not test all possible combinations of compounds in partially ripe mixtures but focused on compounds that showed large differences between ripe and unripe fruits.

\subsubsection{Behavioral test}

We assessed the olfactory discrimination performance of the spider monkeys using a foodrewarded two-choice instrumental conditioning paradigm (Hernandez Salazar et al. 2003). The test apparatus consisted of a $50 \mathrm{~cm}$ long and $6 \mathrm{~cm}$ wide metal bar with two cube-shaped opaque PVC boxes with a side length of $5.5 \mathrm{~cm}$ attached to it at a distance of $22 \mathrm{~cm}$ from each other. Each container was equipped with a tightly closing hinged metallic lid, hanging $2 \mathrm{~cm}$ down the front of the container. From the center of the front part of the lid, a pin of $3 \mathrm{~cm}$ length extended towards the animal and served as a lever to open the lid. On top of each lid was a metal clip attached. This clip held a $70 \times 10 \mathrm{~mm}$ absorbent paper strip (Schleicher \& Schuell, Einbeck, Germany) which was impregnated at its distal end with $10 \mu \mathrm{l}$ of an odorant used as rewarded stimulus (S+) or with $10 \mu \mathrm{l}$ of an odorant used as unrewarded stimulus (S-). The paper strips extended approximately $3 \mathrm{~cm}$ into the cage when the apparatus was presented to the animals. The box with the absorbent paper strip bearing the $S+$ attached to the lid contained a food reward, a Kellogg's Honey Loop $®$, while the one bearing the S- did not. 
Use of fruit odor for fruit choice in black-handed spider monkeys - Methods

When presented with the test apparatus the monkeys sniffed both paper strips for as long as they liked and then decided to open one of the boxes. In the rare cases when a monkey tried to open a box without prior sniffing or tried to open both boxes, the experimenter held a chain connected to the lid tight so that the animal could not move the lid. After the decision and, in the case of a correct choice, after food retrieval the apparatus was immediately removed and prepared for the next presentation out of sight from the monkeys. Each monkey received three blocks of 10 trials (i.e., three sessions) per day. In five of the 10 trials of a session, the left box was baited and in the other five trials the right box was baited. The order of the "correct" and the "wrong" sides was pseudorandomized with the limitation that one box was not baited more often than three times in a row. At the end of each session the apparatus was thoroughly cleaned with $96 \%$ ethanol to ensure that no traces of odorants were left.

Control tests without a food reward being present in the box bearing the absorbent paper strip with the S+ resulted in the same high level of correct choices as tests with a food reward being present in the box. Further, previous studies have shown that the animals consistently failed to perform above chance level when the $S_{+}$was presented at subthreshold (i.e. undetectable) concentrations, despite a food reward being present in the box bearing the absorbent paper strip with the S+. Together, this excludes the possibility that the monkeys smelled the food reward inside the box or based their decisions on cues other than the odors of the $\mathrm{S}+$ and the $\mathrm{S}$-.

The animals were tested individually to avoid distraction from conspecifics. To this end, an animal voluntarily entered a small test cage $(80 \times 50 \times 50 \mathrm{~cm})$ adjacent to the group enclosure which could be closed by a sliding door for temporary separation. The animal sat on a bar mounted horizontally and parallel to the front side of the test cage. This front side of the test cage consisted of a stainless steel mesh with a width of $1 \mathrm{~cm}$ and had two openings of $5 \times 5 \mathrm{~cm}$ allowing the animal to reach through the mesh, open the lid of one of the boxes of the test apparatus and to retrieve the food reward. The test apparatus could be attached to the outside of the front side of the test cage in such a way that the lids of the boxes were at a height consistent with the reachthrough openings. 
We assessed the ability to discriminate between the odors of ripe and unripe fruits, or between the odors of ripe and partially ripe fruits, by assigning one odor mixture mimicking the ripe fruit odor as the rewarded stimulus $\left(\mathrm{S}_{+}\right)$, and several other odor mixtures representing different degrees of unripe fruit odor as the unrewarded stimulus (S-). In order to allow animals to build a robust association between a given odorant and its reward value, the critical tests started by assessing the ability to discriminate between a ripe fruit odor as $\mathrm{S}_{+}$and the monomolecular odorant anethole (described by humans as smelling of aniseed) as S-.

With each stimulus combination, each spider monkey performed six sessions of 10 trials. The first three sessions were considered as training sessions intended to allow the animals to learn the differing reward values of the two stimuli, and the last three sessions were considered as critical sessions that were used for statistical analysis of discrimination performance. Data collection took place between May and September 2014. The spider monkeys were not maintained on a food deprivation schedule but were tested in the morning prior to the presentation of their daily ration of food.

\subsubsection{Experiments}

\section{Tab. 4.1 - Couma macrocarpa - intact fruits}

\begin{tabular}{|c|c|}
\hline & Full ripe vs. \\
\hline 1 & Anethole \\
\hline \multirow[t]{3}{*}{2} & Full unripe odor \\
\hline & Partially ripe odors \\
\hline & Terpenoids: \\
\hline 3 & Unripe $+(E-)$ Caryophyllene \\
\hline 4 & Unripe + a-Copaene \\
\hline 5 & Unripe + monoterpenes (E-B-Ocimene, D-Limonene, Myrcene, Sabinene, $\mathrm{\gamma}$-Terpinen) \\
\hline 6 & Unripe + sesquiterpenes ( $\alpha$-Humulene, $(E-)$ Caryophyllene, $\alpha$-Copaene) \\
\hline
\end{tabular}


Use of fruit odor for fruit choice in black-handed spider monkeys - Methods

\begin{tabular}{|l|l|}
\hline 7 & $\begin{array}{l}\text { Unripe + monoterpenes + sesquiterpenes (E- } \beta \text {-Ocimene, D-Limonene, Myrcene, Sabinene, } \mathrm{V} \text {-Terpinen, } \alpha- \\
\text { Humulene, }(E-) \text { Caryophyllene, } \alpha \text {-Copaene) }\end{array}$ \\
\hline 8 & Aromatic compounds and aldehydes: \\
\hline 9 & Unripe + Ethyl salicylate \\
\hline 10 & Unripe + aromatic compounds (Ethyl salicylate, Methyl salicylate, p-Cymene) \\
\hline 11 & Unripe + Trans-2-nonenal \\
\hline 12 & Unripe + aldehydes (Trans-2-nonenal + Nonanal) \\
\hline 13 & Unripe + aromatic compounds + aldehydes (Ethyl salicylate, Methyl salicylate, Trans-2-nonenal, Nonanal) \\
\hline
\end{tabular}

Tab. 4.2 - Leonia cymosa - intact fruits

\begin{tabular}{|l|l|}
\hline & Full ripe vs. \\
\hline 1 & Anethole \\
\hline 2 & Full unripe \\
\hline 3 & Water \\
\hline
\end{tabular}

Tab. 4.3 - Leonia cymosa - open fruits

\begin{tabular}{|l|l|}
\hline & Full ripe vs. \\
\hline 1 & Anethole \\
\hline 2 & Full unripe \\
\hline & Partially ripe odors \\
\hline & Aromatic compounds: \\
\hline 3 & Unripe + Acetophenone \\
\hline 4 & Unripe + Benzaldehyde \\
\hline 5 & Unripe $+p$-Cymenene \\
\hline 6 & Unripe + aromatic compounds (Acetophenone, Benzaldehyde, Cumene, $p$-Cymene, $p$-Cymenene) \\
\hline & Terpenoids: \\
\hline 7 & Unripe + $\alpha$-Copaene \\
\hline 8 & Unripe + E- $\beta$-Ocimene \\
\hline
\end{tabular}




\begin{tabular}{|c|c|}
\hline 9 & Unripe + all terpenoids ( $E$ - $\beta$-Ocimene, $\alpha$-Copaene $)$ \\
\hline & Monoterpenes + aromatic compounds: \\
\hline 10 & $\begin{array}{l}\text { Unripe + all aromatic compounds and monoterpenes (Acetophenone, Benzaldehyde, Cumene, } p \text {-Cymene, } \\
p \text {-Cymenene, } E-\beta \text {-Ocimene) }\end{array}$ \\
\hline
\end{tabular}

\subsubsection{Data analysis}

For each individual animal, the percentage of correct choices from 30 trials per stimulus combination was calculated. Correct choices consisted both of animals opening a box equipped with the S+ and failing to open a box equipped with the S-. Conversely, errors consisted of animals opening a box equipped with the S- or failing to open a box equipped with the S+. Significance levels were determined by calculating binomial z-scores from the number of correct and false responses for each individual and condition. All tests were two-tailed and two different alpha levels were considerd: 0.05 , corresponding to 21 out of 30 decisions $(=70 \%)$ correct, and 0.01 , corresponding to 23 out of 30 decisions $(=76.7 \%)$ correct.

To assess whether discrimination performance within species/condition (e.g. C. macrocarpa, intact fruits) differed between treatments, we conducted a one-way non-parametric repeatedmeasures ANOVA (Friedman test). If the result of this test proved to be significant or approached significance, we further applied a post-hoc analysis of pairwise non-parametric repeatedmeasures Wilcoxon signed-rank tests, whose p-values were then subjected to the Bonferroni correction for multiple testing. 


\section{SYNOPSIS}

Chapter 2 raised the questions (a) "which functions the sense of smell fulfills in the process of primate food acquisition?" and (b) "which ecological niches favor higher reliance on olfaction when searching and selecting feeding items?". Its conclusions were that available studies are still not fully representative and integratable, but that some patterns emerge. Frugivory probably requires more reliance on olfaction than insectivory or folivory, and this is fully realized only in the context of food selection, and not detection of feeding trees over long distances. Thus, the main function of primate olfaction is recognizing, after the arrival to a fruiting tree, which fruits are ripe and which are unripe, or possibly over-ripe or infested.

$\underline{\text { Chapter } 3}$ examined the implications of this aspect of primate sensory ecology on the evolution of fruit traits. Plant fitness is expected to increase if a reliable signal for fruit ripeness is provided and in turn promotes consumption and consequent seed dispersal by the frugivore. Thus, under the Dispersal Syndrome Hypothesis, in the communication with primates, unique odor indicative of a fruit's ripeness level is expected to be selected for in primate-dispersed species.

However, fruit odor could have functions other than mediating the communication with seeddispersal vectors. Indeed, it might not have a clear function at all. So, to test the hypothesis that fruit odor in primate-dispersed species evolved as a signal for ripeness, a comparative study examined patterns of odor release in four Neotropical species, two dispersed by primates (Couma macrocarpa: Apocynaceae; Leonia cymosa: Violaceae) and two by small passerines (Psychotria cincta: Rubiaceae; Maieta guianensis: Melastomataceae). Passerines are assumed to rely less on olfactory cues and therefore to generate weaker to non-existent selection pressures for the production of an odor signal for ripeness. It set to test three predictions that derive from the hypothesis that fruit odor is an adaptation to primate seed dispersal: (c) that primate-dispersed fruits emit odors which are relatively strong and rich, and most importantly unique to the ripe phase - i.e. provide reliable information that the fruits are ripe; (d) that bird-dispersed species have poorer, weaker odor profiles indistinguishable from the odors of unripe fruits; and (e) that this is independent of phylogeny. 
To test these predictions, chemical analyses of the odor profiles of fruits of the four plant species were conducted. The odor of ripe and unripe fruits was sampled in two conditions, intact and open, that corresponded to two potential signaling mechanisms: husk (intact fruits) and pulp (open fruits) odor. The results confirmed the three predictions: ripe primate-dispersed fruits produced that were significantly different than those of conspecific unripe fruits (C. macrocarpa: in both intact and open conditions; L. cymosa: open only). Bird-dispersed fruits, each phylogenetically closer to one primate-dispersed species than they are to one another, emitted odors that were indistinguishable from those of unripe fruits. Thus, the chapter concluded that patterns of odor release in these four species are in line with the hypothesis that fruit odor in primate-dispersed species is an evolved signal as predicted by the Dispersal Syndrome Hypothesis.

But even if patterns of odor release are in agreement with the predictions of the odor as an adapted signal hypothesis, to demonstrate that fruit odor is indeed a part of an evolved communication system, it was imperative to demonstrate that primates "understand" the signal, i.e. that (f) they can physiologically discriminate between the odors of ripe and unripe primatedispersed fruits, and (g) use this information to select ripe fruits in the absence of cues from other sensory trajectories.

The bioassays reported in chapter 4 addressed these questions and showed that black-handed spider monkeys' (Ateles geoffroyi) behavior was in agreement with the discrimination potential inferred from the chemical analyses presented in chapter 0. In C. macrocarpa, whose odor profiles were rich, strong and different already in the intact phase, the monkeys easily discriminated between the odors of intact ripe and unripe fruits and quickly learned to use the information to correctly choose ripe over unripe fruits in the absence of cues from other sensory trajectories. In L. cymosa primate behavior was also well predicted by the differences of chemical profiles of ripe and unripe fruits. In the intact condition, in which ripe fruit odor was weak and indistinguishable from the odor of unripe fruits, discrimination capacity of the monkeys was low. In contrast, in the open condition, in which ripe fruits emitted strong, rich odors significantly different from that of unripe fruits, spider monkeys easily discriminated between the odors and showed that they can 
rely solely the olfactory signature of the fruits to identify ripe fruits. This positively answered questions ( $\mathrm{f})$ and $(\mathrm{g})$ and confirmed that primates indeed "understand" the signal provided by the primate-dispersed fruits.

Thus, acknowledging that the small model system of four species investigated here should be extended, all predictions of the hypothesis that fruit odor is an evolved signal for seed-dispersing primates have been met. Further, these results provide additional evidence supporting the Dispersal Syndrome Hypothesis, which predicts that fruit traits evolve in response to the anatomical, physiological and sensory capacities of their major dispersal vector.

Finally, an additional set of experiments explored whether any of the compounds or compound classes present in the odor bouquets of primate-dispersed fruits are particularly important in enhancing primates' discrimination capacity between odors of ripe and unripe fruits (question h). The results were negative: no single compound or compound class is, in itself, crucial for creating the perceived difference between the odors of ripe and unripe fruits. 


\section{GENERAL DISCUSSION}

\subsection{The evolution of fruit odor}

Fruit secondary metabolites may have many functions (Cipollini and Levey 1997), some of them non-adaptive (Eriksson and Ehrlén 1998). Recent studies have focused on the long-untested prediction, under the Dispersal Syndrome Hypothesis, that fruits dispersed by olfactorydependent animals such as bats would be subjected to selection pressures to provide a reliable olfactory signal for ripeness. The data presented here joins these findings in showing that fruits dispersed by primates are likely to have been subjected to similar selection pressures and evolved to emit odor signals when ripe. It thus expands the fruit odor an adapted signal model, for the first time, to plants and dispersal vectors outside the bat-fig model system. Furthermore, they provide support for the Dispersal Syndrome Hypothesis by showing that fruit odor is likely to be yet another fruit trait that has been shaped by the sensory capacities of the plants' major seed-dispersal vector.

Potentially, fruit odor could serve two separate functions in the communication with primates: first, to guide them to the fruiting tree and second, to signal whether an individual fruit is ripe or not. However, as noted in chapter 2 , the ability of primates to track odor plumes to detect feeding items over long distances is all but absent. Therefore, as apparent in the results here, the selection pressure that the primate sense of smell exerted on fruit odor has been to emit an odor that characterizes ripe fruits and allows primates to discriminate between ripe and unripe fruits - thus helping them in the selection rather than the foraging phase of food acquisition.

In this context, it is not surprising that the compound classes dominating fruit odor in primatedispersed species are monoterpenes and sesquiterpenes. Terpenoids are heavier and less volatile than many other common volatile organic compounds and are therefore more efficient in signaling quality from short distances than location over long distances (Schaefer and Ruxton 2011). Additionally, chapter 2 concluded that as opposed to the common notion, there is no clear tradeoff between olfaction and color vision in primates. Trichromatic vision, which is either habitual 
or present in some individuals in all haplorrhines and many strepsirrhine species (Jacobs 2009), has been specifically speculated to be useful for detection of fruiting tree from a long distance (Melin et al. 2014). Taken together, this may indicate that fruit bimodal signaling - through color and odor - has evolved to fulfill two different functions: color promotes conspicuousness to primate groups over longer distances whereas odor signals the quality of individual fruits. This idea is further supported by the fact that primate-dispersed fruits are, in the eyes of primates, visually less conspicuous than bird-dispersed fruits (Lomáscolo and Schaefer 2010). This suggests that primate dispersed-species are under a milder selection pressure to be visually conspicuous - possibly because olfactory cues can provide reliable information at the fruitselection stage.

When expanded to the interactions of other fruit species and non-primate frugivores, patterns of odor signaling are expected to be somewhat different. Not surprisingly, all works that discussed the evolution of fruit odor in bat-dispersed species (Hodgkison et al. 2007, 2013; Borges et al. 2008; Lomáscolo et al. 2010) focused solely on husk odor. This is because bat and primate foraging behaviors are different. First, bats lack manual capacities and are not likely to manipulate a fruit and be exposed to the pulp odor before carrying the fruit away. Therefore, bats would not be able to choose a fruit correctly unless a reliable signal is provided without its manipulation in situ. Second, bats rely on olfaction in the search phase as well (Korine and Kalko 2005). Thus, it is likely that bat-dispersed fruits would be under selection pressure to provide olfactory signals that are carried farther away and enhance the long-distance detectability of the fruiting tree, not only the ripeness of individual fruits.

The results from the second set of bioassays (question $h$ ) indicate that not all secondary metabolites emitted by the two primate-dispersed species are necessary for achieving the goal of allowing primates to identify that a fruit is ripe. This leads to an inevitable question: why should fruits synthesize a score of unnecessary compounds?

One potential explanation is that the olfactory signal contains information that is not merely whether the fruit is ripe or not. The underlying working hypothesis in this project has been that the 
signal was binary: simply ripe or unripe, like the one argued to be present in bat dispersed fruits (Hodgkison et al. 2007, 2013; Borges et al. 2008; Lomáscolo et al. 2010). In contrast, the signal's content can, in addition to the information that the fruit is ripe, also provide information regarding fruit nutrient content. Dispersal vectors can be a limiting factor, which leads to inter- and intraspecific competition for seed dispersers (Alcántara et al. 1997). Thus, individual plants are under pressure to provide an attractive reward to seed dispersers, but also to provide reliable signals regarding the quality of the fruits they offer. Frugivores often need such sensory cues to assess the quality of fruits. Primates may rely on texture or ethanol content as a proxy for sugar content (Dominy 2004b), and birds use fruit color, which is assumed to be an evolved signal to birds similarly to odor in primate-dispersed species. Crucially, fruit color in bird-dispersed species is correlated with fat content and thus provides a reliable signal for fruit quality (Schaefer et al. 2014).

Therefore, the function of the rich odor bouquets emitted by C. macrocarpa and L. cymosa may be to signal fruit quality, and not merely that the fruits are ripe. In fact, most fruit volatiles are biosynthetically associated with nutrients and therefore provide reliable cues for fruit quality (Goff and Klee 2006). Further, the compound class that dominates the odors of intact $C$. macrocarpa and open L. cymosa fruits is terpenoids, whose main building block is isoprene (McGarvey and Croteau 1995), a compound whose emission from leaves is correlated with photosynthetic activity (Lerdau and Throop 2000). Thus, it is plausible that individual compounds in the bouquet are cues for the presence of specific nutrients, and that a terpenoid rich volatile bouquet is a reliable, "honest" (sensu Zahavi 1975; Zahavi and Zahavi 1997), signal for sugar content and fruit quality. This hypothesis can be confirmed if future studies show that terpenoid richness is indeed positively correlated with sugar content and that primates show a preference for fruits that emit terpenoid-rich bouquets.

Other, less adaptive explanations may account for the excess of volatile compounds emitted by the two primate-dispersed species. Plant secondary metabolic biosynthetic pathways are typically non-specific: rather than synthesizing one compound, they produce several similar-yet-different 
compounds (Fischbach and Clardy 2007). While it is unclear whether this has any adaptive function or whether it derives from a constraint that prevents fully specific biosynthesis, the inevitable result is that plant secondary compounds are often present in mixtures. As a result, fruits that are under selection to use secondary metabolites as olfactory signals for seed dispersers are forced, due to this constraint, to present an overly complex mixture.

Finally, it should be noted that by focusing on the role of fruit secondary metabolites in communication with primates, the scope taken in this thesis is, admittedly, rather narrow. The many potential functions of fruit secondary compounds (Cipollini and Levey 1997), along with inevitable constraints, generate multiple selection pressures in varying directions (Borges 2015). Consequently, the discussion of the evolution of fruit odor should rather explore the evolution of fruit odors, not odor. In this context, it is far from surprising that fruit odor contained compounds that are unnecessary for discrimination between ripe and unripe fruits: synthesis of some of the compounds identified may have evolved to fulfill other functions, or may be present due to pleiotropic constraints (i.e. their synthesis in other plant organs, namely leaves). Therefore, although the trends documented here indicate that the need to signal ripeness to primates indeed exerted significant selection pressures that resulted in differences in patterns of odor release between primate- and bird-dispersed species, the main task of future research addressing this is to identify which components of fruit odor responded to which selection pressures.

\subsection{Primate olfaction: functions and evolution}

Just like the synthesis of fruit secondary metabolites is the result of many, differing, selection pressures combined with phylogenetic and developmental constraints (Borges 2015), so is the primate sense of smell: factors like activity time, modal diet and the availability of cues from other sensory trajectories join constraints to form species-specific olfactory capacities and overall level of reliance on olfaction. Thus, chapter 2 concluded that more systematic comparative studies of primate behavior are required to pinpoint large-scale evolutionary scenarios in primate olfactory 
evolution. However, available behavioral works indicate that frugivory requires more reliance on olfactory cues than other dietary categories and that the function of olfaction in this context is fruit selection. It further speculated that the particular olfactory capacity that is expected to be crucial for fulfilling this function is olfactory discrimination, i.e. the ability to tell that two odors are different and thus also to identify odors, and not olfactory sensitivity - the ability to detect odorants at low concentrations.

The data presented in chapters 3 and $0 \underline{4}$ are in line with these trends and predictions. Spider monkeys proved to be able to easily discriminate between odors of ripe and unripe fruits with which they had been previously unfamiliar, and to be able to use this ability to correctly choose ripe fruits almost immediately. Furthermore, they could easily discriminate the odors of ripe and unripe fruits even when the latter were manipulated to be increasingly more similar to the former. This indicates that primates do not rely on any individual compound for odor identification and that their olfactory system is acute and flexible enough to filter out a lot of noise and still identify stimuli. These results raise the discussion regarding the relative importance of olfaction to primate feeding ecology to a whole new level: if olfaction did not play a crucial role in primate feeding behavior, it could not exert any significant selection pressures on the consumed fruits and the patterns of fruit odor production would probably be very different.

The work reported here provides the first detailed account of realistic chemical challenges with which the primate sense of smell has to deal in real-life ecological challenges. Based on only two plant species, the data presented here regarding this "olfactory space" is still limited. However, it is clear that the chemical signal identified in both primate-dispersed species contains a significant share of terpenoids. Terpenoids are by far the most important family of plant volatile compounds (Gershenzon and Dudareva 2007) and the ability to detect them and discriminate between them should be useful, apart from frugivory, in all chemically-mediated primate-plant interactions. Thus, future studies that wish to identify aspects of primate olfaction that evolved for food selection should focus on measurements of olfactory sensitivity and discrimination capacities to terpenoids and other compounds identified in fruit odor. 
One question left unanswered by the data presented here is the level to which primate response to fruit olfactory signals is innate or learned. In insect-plant pollination interactions, which are in many cases much more specific than frugivore-plant interactions (Blüthgen et al. 2007), chemical communication is often based on a small number of compounds and the elicited insect behavior is often hard-wired (i.e. it is innate and does not require learning) (Lunau 1992). In contrast, primates can consume hundreds of different fruit species (van Roosmalen 1985b,Julliot 1996b; Culot 2009), some of which provide fruits only every other year (Chapman et al. 2005). Although there is no information regarding their volatile profiles, data from cultivated fruit species suggest that the diversity of fruit volatiles is enormous (McGrath and Karahadian 1994; Beaulieu and Grimm 2001; Franco and Janzantti 2005). Thus, the chemical stimuli primates deal with when choosing fruits are likely to be highly complex and unpredictable - a condition that strongly promotes plastic, learned, responses rather than innate reactions.

On the other hand, innate response to predator-related odorants has been documented in mouse lemurs (Microcebus murinus) (Sündermann et al. 2008) and even in birds, whose web of interactions with fruits is as complex as that of primates, innate response to visual fruit stimuli coexists alongside learned behavior (Schaefer and Ruxton 2011). Additionally, the previous section ( $\underline{6.1})$ speculated that some volatile compounds - especially terpenoids - may be inherently associated with sugar content. Although learned discrimination between odors of ripe and unripe fruits may still be required, in this context some innate preference for terpenoid-rich odors may be selected. Thus, it cannot be excluded that at least some of primates' "understanding" of fruit chemical signals is innate. Unfortunately, the experiments described in chapter 4 cannot shed light on this question because the experimental procedure required conditioning of the subjects before the experiments begun. Therefore, all they can tell us is that even without innate response, primates can learn to use olfactory cues to choose ripe fruits. Based on these results, it is impossible to tell whether any innate preference was present in the monkeys. 
General discussion - "Chemical coevolution" of primates and angiosperms?

\section{3. "Chemical coevolution" of primates and angiosperms?}

The major radiations of primates and angiosperms coincided (Eriksson 2014) and the two groups show a lot of co-dependency: many primate species of all lineages show at least some degree of frugivory and angiosperms, especially in the tropics, rely on their seed-dispersal services (see 1.2.1). These observations have led to the "primate-angiosperm coevolution hypothesis", which postulates that the major drive behind the early evolution of primates was their interactions with the co-evolving flowering plants (Sussman 1991; Sussman et al. 2013). Support for this notion is somewhat circumstantial: apart from the temporal coincidence and contemporary mutualistic interactions, it strongly relies on a single fossil (Bloch and Boyer 2002). So, coevolution sensu stricto - i.e. a tight process in which gradual changes in one taxon drive a change in another, which in turn drives further change in the former - might not best describe the complex, long-term and, most importantly, non-exclusive relationship between primates and angiosperms (Janzen 1980).

However, several angiosperm and primate traits can clearly be attributed to the interaction with one another: the patterns of fruit production and spatial distribution have had a profound effect on primate social evolution (Wrangham 1980) and primate-dispersed fruits tend to have larger seeds and fruits as well as a thick husk (Janson 1983; Howe 1986), which makes them better protected and more viable but less accessible to many other frugivores.

The chemical communication described here may represent yet another aspect of primate-plant shared evolutionary history. The presence of unique odor marking ripe fruits of only primatedispersed fruits, independent of phylogeny and used by monkeys to identify ripeness, strongly indicates that the plant side of the interaction is shaped by the interaction with primates. Whether or not primate olfactory evolution has been shaped by the chemical communication with plants more difficult to answer with the data presented here. However, the quasi-comparative review of behavioral data (chapter 2) indicated that frugivory is the dietary category which requires and can benefit the most from olfaction and physiological studies showed that the primate sense of smell is particularly tuned to detect and discriminate fruit-related odorants (Ueno 1994,Laska and Seibt 
General discussion - "Chemical coevolution" of primates and angiosperms?

2002a; Hernandez Salazar et al. 2003,Laska et al. 2006a,b). Thus, although not based on data presented here, it seems safe to assume that fruit odor has shaped primate olfactory evolution as well.

Nonetheless, whether or not we can identify selection pressures acting on both sides of the primate-plant interactions, this does not imply that fruit odor or primate relevant olfactory capacities have evolved de novo as a direct response to the interaction with one another. Due to differences in generation length and to the fact that both plants and frugivores are generalists, tight species-to-species coevolutionary processes between plant and seed disperser are very unlikely (Herrera 1985). The more likely scenario is of softer, diffuse, coevolution (Janzen 1980; Herrera 1985): fleshy fruits evolved independently in many lineages (Bremer and Eriksson 1992; Bolmgren and Eriksson 2005; Eriksson 2014) and, due to the benefits of producing larger seeds and a thick-husked protected fruit (see 1.1.1), converged to produce the typical "mammal dispersal syndrome" fruits (Janson 1983; Link and Stevenson 2004) and rely on the dispersal services of relatively larger, arboreal, mammals. The taxa that filled this ecological niche may have been different in various ecological systems and time periods: early "mammal fruits" may have relied on dispersal by frugivorous multituberculates and later ones may have evolved when rodents, plesiadapiforms or early euprimates filled this niche (Eriksson 2014). As long as selection pressures exerted by the occupants of this niche were consistent and directional, evolution of fruit traits in response is expected (Janzen 1980; Herrera 1985).

Olfaction is a major sensory modality in most mammals (Ache 1991) and since primates are now known to share this olfactory acuity with other mammals (e.g. Laska et al. 2000), olfactory signaling of fruit ripeness is expected to be a part of the mammalian dispersal syndrome in many systems. Indeed, it is impossible at this point to determine whether the olfactory signals described here have originated as a result of selection pressures exerted by primate seed dispersal or whether it was the interaction with other similar mammals. But even if primates radiated only later into the olfactory-guided arboreal frugivorous mammal niche, the fact that fruit odor still mediates mutually beneficial communication with the contemporary occupants of this niche implies that they 
are likely to have continued generating these same selection pressures. Thus, even if olfactory signals originated when interacting with other species, their function in communicating with primates drives plants to retain olfactory signaling in the sense of "use it or lose it".

\section{4. $\quad$ Caveats}

Although the data presented are in line with the predictions generated by the hypothesis that fruit odor has evolved as a communication system with seed dispersers, the study suffers from several limitations that should be addressed before generalizations to primate-plant interactions as a whole can be made. First, the four model plant species are relatively far-related. This was inevitable since the selection of model species had to be done in field conditions because it had been impossible to predict which species would provide fruits in sufficient amounts. Within family plant taxonomy is still frequently revisited and even in families in which the phylogeny is considered to be resolved, there was a risk of wrong identification in the field. Either of the two wrong identification or a major revision of the family's phylogeny - could render the results of the study meaningless if the control for phylogeny, i.e. the fact that primate-dispersed species are each phylogenetically closer to one bird-dispersed species than they are to one another, were lost. In principal, the fact that species are relatively far-related does not weaken the conclusions because patterns of odor release in the four species are still independent of phylogeny. Yet the farther species are, the more changes in the relevant traits in either direction could have taken place since their last common ancestor. Thus, conducting a similar study on a smaller scale within family or even genus - could be more informative.

Second, due to time and budget constraints, sample sizes were rather small. The model system of four species is clearly too small to determine whether the patterns described here can indeed be generalized to the entire range of fruit-bearing plants dispersed by primates, in the Neotropics and beyond. Less problematic, given the consistent results and the standards in the field, the five individuals available for the bioassays ( $\underline{\text { chapter } 4)}$ can be considered a reasonable sample for the 
General discussion - Concluding remarks: towards an evolutionary chemical ecology of primate plant interactions

physiological experiments conducted. Yet it would clearly be beneficial to repeat them with more individuals - preferably from other primate species, and possibly not in captivity.

Finally, the bioassays that tested primate reaction to fruit odor used synthetic mixtures instead of natural odors. This allowed presenting standardized mixtures to all participants. Further, the additional set of experiments which examined the roles of individual compounds in primate discrimination capacity could be conducted only when stimuli are synthetic. Yet while the synthetic mixtures reasonably resembled the odor of natural fruits (see appendix 2), they did not contain all identified compounds and they could not reflect the natural variation in the odor of fruits from the same species and ripeness level. Thus, although using synthetic mixtures has had many benefits, the conclusions drawn from the experiments would be strengthened if they are replicated using odors of natural fruits.

\subsection{Concluding remarks: towards an evolutionary chemical ecology of primate plant} interactions

Chemical ecology - the study of the role of chemical compounds in mediating interactions between organisms - is a well-established interdisciplinary field. Among its model systems, insectplant interactions have taken a prominent role while work with vertebrates has been rarer. Primates, whose sense of smell had been considered almost negligible up until the turn of the new century, have only recently begun receiving attention. The majority of work has focused on intraspecific communication, but by now there are numerous studies describing the roles of chemosensation in primates' interaction with their environment, too. However, as noted in chapters $\underline{2}$ and $\underline{4}$, integration of works has been limited. Physiological studies documented olfactory sensitivity and discrimination capacities for a myriad of ecologically irrelevant stimuli, and behavioral works ignored primate olfactory physiology and as a result could not conclude much beyond a general notion that primates can sometimes use their sense of smell. The gap that lies between them is the lack of any knowledge regarding the chemical world with which the primate 
General discussion - Concluding remarks: towards an evolutionary chemical ecology of primate plant interactions

sense of smell interacts, which can be used to connect primate and plant physiology and thus to explore how they interact on the proximate level and how they may have affected the evolution of one another.

The current study provides a first attempt to fill this gap by importing methods and approaches from chemical ecology into the study of non-human primates. It provides the first detailed chemical analyses of the odor of primate-consumed fruits alongside bioassays that measured the ability of primates to use a clearly defined physiological capacity (discrimination) to solve real-life feeding tasks. The comparative approach employed in chapter 3 puts this interaction in an evolutionaryecological context. On the other hand, as mentioned above (6.46.4), as a first step in this direction it still suffers from several limitations that qualify its conclusions.

Furthermore, the patterns described here are only a private case: two Neotropical primatedispersed species. Different ecological systems have experienced different evolutionary histories that are expected to yield convergence of patterns in some and divergence in others. For example, in continental Africa primates and birds overlap in their sensory capacities (Jacobs 2009) and the fruit species on which they feed (Gautier-Hion et al. 1985). This predicts that chemical communication between primates and plants would turn out to be less significant in this system. On the other hand, in Madagascar lemurs play a much more important role in seed dissemination and their sensory systems are closer to those of Neotropical primates. So, it is predicted that patterns of primate-plant chemical communication would be similar to those implied for the Neotropics in this study.

Thus, rather than providing final, full, answers, the current study should serve as a starting point for an investigation of the roles of fruit secondary compounds in mediating primate-plant interactions. The discussion in each chapter offered many questions for future studies and raised concerns regarding potential confounding factors. Rather than repeating them here, Fig. 6.1 provides a synthesis of the approach taken in this thesis alongside the many open questions. It schematically portrays the myriad of questions and relevant factors to the investigation of the functions and evolution of chemically-mediated primate plant interactions and thus attempts to 
General discussion - Concluding remarks: towards an evolutionary chemical ecology of primate plant interactions

provide a framework for future studies of both proximate mechanisms and ultimate functions of primate-plant chemical communication.

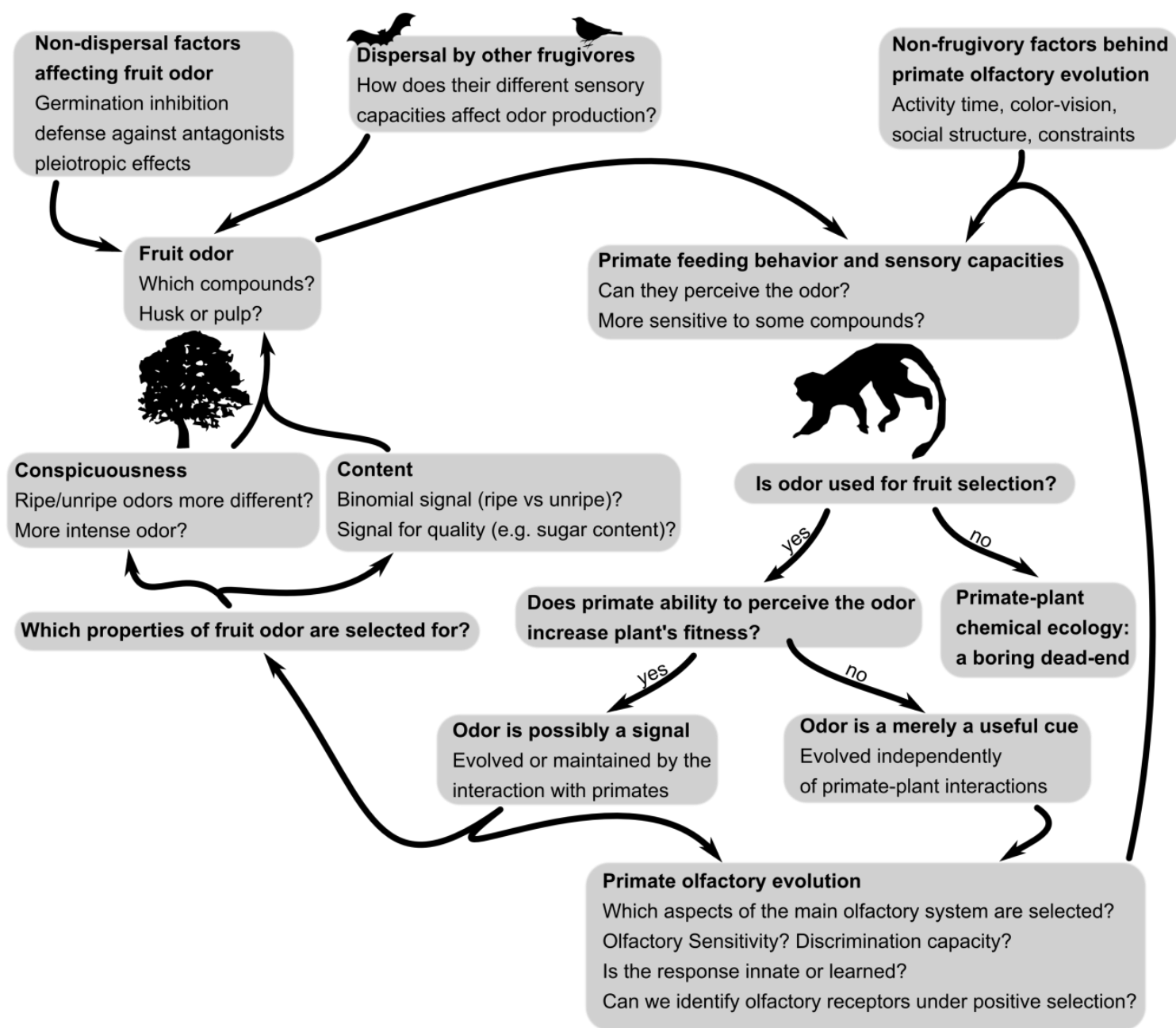

Figure 6.1. Towards an evolutionary chemical ecology of primate-plant interactions. A synthesis of questions, approaches and potential confounding factors to investigate the evolution and ecological roles of chemical signals in primate-plant interactions. 


\section{REFERENCES CITED}

Ache, B. W. 1991. Phylogeny of smell and taste. Pp. 3-18 in T. V. Getchell, R. L. Doty, L. M. Bartoshuk, and J. B. Snow, eds. Smell and Taste in Health and Disease. Raven Press, New-York.

Adams, R. P. 2007. Identification of Essential Oil Components by Gas Chromatography/Mass Spectrometry. 4th ed. Allured Pub. Corp., Carol Streams, IL.

Alcántara, J. M., P. J. Rey, F. Valera, A. M. Sánchez-Lafuente, and J. E. Gutiárrez. 1997. Habitat alteration and plant intra-specific competition for seed dispersers. An example with Olea europaea var. sylvestris. Oikos 79:291-300.

Ankel-Simons, F. 2007. Primate Anatomy. 3rd ed. Academic Press, San-Diego.

Anzures-Dadda, A., E. Andresen, M. L. Martínez, and R. H. Manson. 2011. Absence of howlers (Alouatta palliata) influences tree seedling densities in tropical rain forest fragments in southern Mexico. Int. J. Primatol. 634-651.

Ballard, H. E., J. De Paula-souza, and G. A. Wahlert. 2014. Violaceae. Pp. 303-322 in K. Kubitzki, ed. Flowering Plants: Eudicots. Springer, Berlin Heidelberg.

Ballard, H. E., and K. J. Sytsma. 2000. Evolution and biogeography of the woody Hawaiian violets (Viola, Violaceae): arctic origins, herbaceous ancestry and bird dispersal. Evolution 54:15211532.

Baron, G., H. D. Frahm, K. P. Bhatnagar, and H. Stephan. 1983. Comparison of brain structure volumes in insectivora and primates. III. Main olfactory bulb (MOB). J. Hirnforsch. 24:551-568.

Barton, R. 2006. Olfactory evolution and behavioral ecology in primates. Am. J. Primatol. 68:545558.

Barton, R., and P. H. Harvey. 2000. Mosaic evolution of brain structure in mammals. Nature 405:1055-1058.

Barton, R., A. Purvis, and P. H. Harvey. 1995. Evolutionary radiation of visual and olfactory brain systems in primates, bats and insectivores. Philos T Roy Soc B 348:381-392.

Baxi, K. N., K. M. Dorries, and H. L. Eisthen. 2006. Is the vomeronasal system really specialized for detecting pheromones? Trends Neurosci. 29:1-7.

Beaulieu, J. C., and C. C. Grimm. 2001. Identification of volatile compounds in cantaloupe at various developmental stages using solid phase microextraction. J. Agric. Food Chem. 49:13451352.

Bennett, A. T. D., and M. Théry. 2007. Avian color vision and coloration: multidisciplinary evolutionary biology. Am. Nat. 169:S1-S6.

Bicca-Marques, J. C., and P. A. Garber. 2004. Use of spatial, visual, and olfactory information during foraging in wild nocturnal and diurnal anthropoids: a field experiment comparing Aotus, Callicebus, and Saguinus. Am. J. Primatol. 62:171-187.

Bloch, J. I., and D. M. Boyer. 2002. Grasping primate origins. Science 298:1606-1610.

Blüthgen, N., F. Menzel, T. Hovestadt, B. Fiala, and N. Blüthgen. 2007. Specialization, constraints, and conflicting interests in mutualistic networks. Curr. Biol. 17:341-346.

Bolen, R. H., and S. M. Green. 1997. Use of olfactory cues in foraging by owl monkeys (Aotus nancymai) and capuchin monkeys (Cebus apella). J. Comp. Psychol. 111:152-158. 
Bollen, A., G. Donati, J. Fietz, D. Schwab, J.-B. Ramanamanjato, L. Randrihasipara, L. van Elsacker, and J. U. Ganzhorn. 2005. An intersite comparison of fruit characteristics in Madagascar: evidence for selection pressure through abiotic constraints rather than through coevolution. Pp. 93-119 in J. L. Dew and J. P. Boubli, eds. Tropical Fruits and Frugivores. Springer, Dordrecht.

Bolmgren, K., and O. Eriksson. 2005. Fleshy fruits - origins, niche shifts, and diversification. Oikos 109:255-272.

Bolmgren, K., and O. Eriksson. 2010. Seed mass and the evolution of fleshy fruits in angiosperms. Oikos 119:707-718.

Borges, R. M. 2015. Fruit and seed volatiles: multiple stage settings, actors and props in an evolutionary play. J. Indian Inst. Sci. 95:93-104.

Borges, R. M., J. M. Bessière, and M. Hossaert-McKey. 2008. The chemical ecology of seed dispersal in monoecious and dioecious figs. Funct. Ecol. 22:484-493.

Borges, R. M., J.-M. Bessière, and Y. Ranganathan. 2013. Diel variation in fig volatiles across syconium development: making sense of scents. J. Chem. Ecol. 39:630-642.

Borges, R. M., Y. Ranganathan, A. Krishnan, M. Ghara, and G. Pramanik. 2011. When should fig fruit produce volatiles? Pattern in a ripening process. Acta Oecologica 37:611-618.

Bremer, B., and O. Eriksson. 1992. Evolution of fruit characters and dispersal modes in the tropical family Rubiaceae. Biol. J. Linn. Soc. 47:79-95.

Buck, L. B. 2004. Olfactory receptors and odor coding in mammals. Nutr. Rev. 62:S184-S188.

Buck, L. B., and R. Axel. 1991. A novel multigene family may encode odorant receptors: a molecular basis for odor recognition. Cell 65:175-87.

Bushdid, C., M. O. Magnasco, L. B. Vosshall, and A. Keller. 2014. Humans can discriminate more than 1 trillion olfactory stimuli. Science 343:1370-1372.

Campbell, C. J., A. Fuentes, and K. MacKinnon. 2010. Primates in Perspective. 2nd ed. Oxford University Press, Oxford.

Cartmill, M. 1974. Rethinking primate origins. Science 184:436-443.

Cazetta, E., M. Galetti, E. L. Rezende, and H. M. Schaefer. 2011. On the reliability of visual communication in vertebrate-dispersed fruits. J. Ecol. 100:277-286.

Cazetta, E., H. M. Schaefer, and M. Galetti. 2007. Why are fruits colorful? The relative importance of achromatic and chromatic contrasts for detection by birds. Evol. Ecol. 23:233-244.

Chapman, C. A. 1995. Primate seed dispersal: Coevolution and conservation implications. Evol. Anthropol. 4:74-82.

Chapman, C. A., T. R. Bonnell, J. F. Gogarten, J. E. Lambert, P. A. Omeja, D. Twinomugisha, M. D. Wasserman, and J. M. Rothman. 2013. Are primates ecosystem engineers? Int. J. Primatol. 34:1-14.

Chapman, C. a., L. J. Chapman, T. T. Struhsaker, A. E. Zanne, C. J. Clark, and J. R. Poulsen. 2005. A long-term evaluation of fruiting phenology: importance of climate change. J. Trop. Ecol. 21:31-45.

Chapman, C. A., and D. A. Onderdonk. 1998. Forests without primates: primate/plant codependency. Am. J. Primatol. 141:127-141. 
Chapman, C. A., and S. E. Russo. 2007. Linking behavioral ecology with forest community structure. Pp. 510-525 in C. J. Campbell, A. Fuentes, K. C. MacKinnon, M. Panger, and S. K. Bearder, eds. Primates in Perspective. Oxford University Press, New-York.

Cipollini, M. L., and D. J. Levey. 1997. Secondary metabolites of fleshy vertebrate-dispersed fruits: adaptive hypotheses and implications for seed dispersal. Am. Nat. 150:346-372.

Clark, C. J., J. R. Poulsen, B. M. Bolker, E. F. Connor, and V. T. Parker. 2005. Comparative seed shadows of bird, monkey-, and wind-dispersed trees. Ecology 86:2684-2694.

Clausing, G., K. Meyer, and S. S. Renner. 2000. Correlations among fruit traits and evolution of different fruits within Melastomataceae. Bot. J. Linn. Soc. 133:303-326.

Connell, J. H. 1971. On the role of natural enemies in preventing competitive exclusion in some marine animals and in rain forest trees. Pp. 298-323 in P. J. den Boer and G. R. Gradwell, eds. Dynamics of Populations. Centre for Agricultural Publishing and Documentation, Wageningen.

Culot, L. 2009. Primary seed dispersal by two sympatric species of tamarins, Saguinus fuscicollis and Saguinus mystax, and post-dispersal seed fate. Université de Liège, DPZ.

Culot, L., D. J. Mann, F. J. J. Muñoz Lazo, M.-C. Huynen, and E. W. Heymann. 2010a. Tamarins and dung beetles: an efficient diplochorous dispersal system in the Peruvian Amazonia. Biotropica 43:84-92.

Culot, L., F. J. J. Muñoz Lazo, M.-C. Huynen, P. Poncin, and E. W. Heymann. 2010b. Seasonal variation in seed dispersal by tamarins alters seed rain in a secondary rain forest. Int. J. Primatol. 31:553-569.

da Costa, R. S., and J. C. Bicca-Marques. 2014. Owl monkeys (Aotus nigriceps and A. infulatus) follow routes instead of food-related cues during foraging in captivity. PLoS One 9:e115188.

Davies, T. J., T. G. Barraclough, M. W. Chase, P. S. Soltis, D. E. Soltis, and V. Savolainen. 2004. Darwin's abominable mystery: insights from a supertree of the angiosperms. P Natl Acad Sci USA 101:1904-1909.

de Sousa, A. A., and M. J. Proulx. 2014. What can volumes reveal about human brain evolution? A framework for bridging behavioral, histometric and volumetric perspectives. Front. Neuroanat. 8:51.

Deaner, R. O., C. L. Nunn, and C. P. van Schaik. 2000. Comparative tests of primate cognition: different scaling methods produce different results. Brain. Behav. Evol. 55:44-52.

di Fiore, A., A. Link, and J. L. Dew. 2008. Diets of wild spider monkeys. Pp. 81-137 in Spider Monkeys: Behavior, Ecology and Evolution of the Genus Ateles. Cambridge University Press, New-York.

Dittus, W. P. J. 1985. The influence of leaf-monkeys on their feeding trees in a cyclone-disturbed environment. Biotropica 17:100-105.

Dominy, N. J. 2004a. Color as an indicator of food quality to anthropoid primates: ecological evidence and an evolutionary scenario. Pp. 615-644 in C. F. Ross and R. F. Kay, eds. Anthropoid Origins: New Visions. Kluwer Academic / Plenum Publishers, New-York.

Dominy, N. J. 2004b. Fruits, fingers, and fermentation: the sensory cues available to foraging primates. Integr. Comp. Biol. 44:295-303.

Dominy, N. J., P. A. Garber, J. C. Bicca-Marques, and M. A. de O. Azevedo-Lopes. 2003. Do female tamarins use visual cues to detect fruit rewards more successfully than do males? Anim. Behav. 66:829-837. 
Dominy, N. J., and P. W. Lucas. 2001. Ecological importance of trichromatic vision to primates. Nature 410:363-366.

Dominy, N. J., and P. W. Lucas. 2004. Significance of color, calories, and climate to the visual ecology of catarrhines. Am. J. Primatol. 62:189-207.

Dominy, N. J., P. W. Lucas, D. Osorio, and N. Yamashita. 2001. The sensory ecology of primate food perception. Evol. Anthropol. 10:171-186.

Dominy, N. J., P. W. Lucas, and N. Supardi Noor. 2006. Primate sensory systems and foraging behavior. Pp. 489-509 in G. Hohmann, M. M. Robbins, and C. Boesch, eds. Feeding Ecology in Apes and Other Primates. Cambridge University Press, Cambridge.

Donatti, C. I., P. R. Guimarães, M. Galetti, M. A. Pizo, F. M. D. Marquitti, and R. Dirzo. 2011. Analysis of a hyper-diverse seed dispersal network: modularity and underlying mechanisms. Ecol. Lett. 14:773-781.

Dong, D., G. He, S. Zhang, and Z. Zhang. 2009. Evolution of olfactory receptor genes in primates dominated by birth-and-death process. Genome Biol. Evol. 1:258-264.

Dötterl, S., and A. Jürgens. 2005. Spatial fragrance patterns in flowers of Silene latifolia: Lilac compounds as olfactory nectar guides? Plant Syst. Evol. 255:99-109.

Dötterl, S., L. M. Wolfe, and A. Jürgens. 2005. Qualitative and quantitative analyses of flower scent in Silene latifolia. Phytochemistry 66:203-213.

Dray, S., and A. B. Dufour. 2007. The ade4 package: implementing the duality diagram for ecologists. J. Stat. Softw. 22:1-20.

Elliot Smith, G. 1927. The Evolution of Man. Oxford University Press, London.

Eriksson, O. 2014. Evolution of angiosperm seed disperser mutualisms: the timing of origins and their consequences for coevolutionary interactions between angiosperms and frugivores. Biol. Rev., doi: $10.1111 /$ brv.12164.

Eriksson, O., and J. Ehrlén. 1998. Secondary metabolites in fleshy fruits: are adaptive explanations needed? Am. Nat. 152:905-907.

Farmer, E. E. 2014. Leaf Defence. Oxford University Press, Oxford.

Felsenstein, J. 1985. Phylogenies and the compartative method. Am. Nat. 125:1-15.

Finlay, B. L., and R. B. Darlington. 1995. Linked regularities in the development and evolution of mammalian brains. Science 268:1578-1584.

Finlay, B. L., R. B. Darlington, and N. Nicastro. 2001. Developmental structure in brain evolution. Behav. Brain Sci. 24:263-308.

Fischbach, M. A., and J. Clardy. 2007. One pathway, many products. Nat. Chem. Biol. 3:353355.

Fischer, K. E., and C. A. Chapman. 1993. Frugivores and fruit syndromes: differences in patterns at the genus and species level. Oikos 66:472-482.

Fleming, A. J. 2005. Formation of primordia and phyllotaxy. Curr. Opin. Plant Biol. 8:53-58.

Flörchinger, M., J. Braun, K. Böhning-Gaese, and H. M. Schaefer. 2010. Fruit size, crop mass, and plant height explain differential fruit choice of primates and birds. Oecologia 164:151-161.

Fobes, J. L., and J. E. King. 1982. Vision: the dominant primate modality. Pp. 219-243 in J. L. Fobes and J. E. King, eds. Primate Behavior. Academic Press, New-York. 
Franco, M. R. B., and N. S. Janzantti. 2005. Aroma of minor tropical fruits. Flavour Fragr. J. 20:358-371.

Galetti, M., R. Guevara, M. C. Côrtes, R. Fadini, S. Von Matter, A. B. Leite, F. Labecca, T. Ribeiro, C. S. Carvalho, R. G. Collevatti, M. M. Pires, P. R. Guimarães, P. H. Brancalion, M. C. Ribeiro, and $P$. Jordano. 2013. Functional extinction of birds drives rapid evolutionary changes in seed size. Science 340:1086-1090.

Gautier-Hion, A., J. M. Duplantier, R. Quris, F. Feer, C. Sourd, J. P. Decoux, G. Dubost, L. Emmons, C. Erard, H. Hecketsweiler, A. Moungazi, C. Roussilhon, and J. M. Tliiollay. 1985. Fruit characters as a basis of fruit choice and seed dispersal in a tropical forest vertebrate community. Oecologia 65:324-337.

Geravis, J. A., B. R. Noon, and M. F. Willson. 1999. Avian selection of the color-dimorphic fruits of salmonberry, Rubus spectabilis: a field experiment. Oikos 84:77-86.

Gershenzon, J., and N. Dudareva. 2007. The function of terpene natural products in the natural world. Nat. Chem. Biol. 3:408-414.

Gilad, Y., O. Man, and G. Glusman. 2005. A comparison of the human and chimpanzee olfactory receptor gene repertoires. Genome Res. 15:224-230.

Gilad, Y., V. Wiebe, M. Przeworski, D. Lancet, and S. Pääbo. 2004. Loss of olfactory receptor genes coincides with the acquisition of full trichromatic vision in primates. PLoS Biol. 2:01200125 .

Gittleman, J. L. 1991. Carnivore olfactory bulb size: allometry, phylogeny and ecology. J. Zool. 225:253-272.

Goff, S. A., and H. J. Klee. 2006. Plant volatile compounds: sensory cues for health and nutritional value? Science 311:815-819.

Gorchov, D. L., F. Cornejo, C. Ascorra, and M. Jaramillo. 1995. Dietary overlap between frugivorous birds and bats in the Peruvian Amazon. Oikos 74:235-250.

Gould, S. J., and R. C. Lewontin. 1979. The spandrels of San Marco and the Panglossian paradign: a critique of the adaptionist programme. Proc. R. Soc. London. Ser. B Biol. Sci. 205:581-598.

Hamrick, J. L., and D. W. Trapnell. 2011. Using population genetic analyses to understand seed dispersal patterns. Acta Oecologica 37:641-649.

Hayden, S., M. Bekaert, T. A. Crider, S. Mariani, W. J. Murphy, and E. C. Teeling. 2010. Ecological adaptation determines functional mammalian olfactory subgenomes. Genome Res. 20:1-9.

Healy, S. D., and T. Guilford. 1990. Olfactory-bulb size and nocturnality in birds. Evolution 44:339346.

Heritage, S. 2014. Modeling olfactory bulb evolution through primate phylogeny. PLoS One 9:e113904.

Hernandez Salazar, L. T., M. Laska, and E. Rodriguez Luna. 2003. Olfactory sensitivity for aliphatic esters in spider monkeys (Ateles geoffroyi). Behav. Neurosci. 117:1142-1149.

Herrera, C. M. 1985. Determinants of plant-animal coevolution: the case of mutualistic dispersal of seeds by vertebrates. Oikos 44:132-141.

Herrera, C. M. 2002. Seed dispersal by vertebrates. Pp. 185-208 in C. M. Herrera and O. Pellmyr, eds. Plant-Animal Interactions: an Evolutionary Approach. Blackwell Scientific Publications, 
Oxford.

Heymann, E. W. 2011. Florivory, nectarivory, and pollination - a review of primate-flower interactions. Ecotropica 17:41-52.

Heymann, E. W. 1995. Sleeping habits of tamarins, Saguinus mystax and Saguinus fuscicollos (Mammalia; Primates; Callitrichidae), in north-eastern Peru. J. Zool. 237:211-226.

Heymann, E. W. 2006. The neglected sense - olfaction in primate behavior, ecology, and evolution. Am. J. Primatol. 68:519-524.

Hilker, M., and J. McNeil. 2008. Chemical and behavioral ecology in insect parasitoids: how to behave optimally in a complex odorous environment. Pp. 92-112 in E. Wajnberg, C. Bernstein, and J. van Alphen, eds. Behavioral Ecology of Insect Parasitoids. Blackwell Scientific Publications, Malden, MA.

Hiramatsu, C., A. D. Melin, F. Aureli, C. M. Schaffner, M. Vorobyev, and S. Kawamura. 2009. Interplay of olfaction and vision in fruit foraging of spider monkeys. Anim. Behav. 77:1421-1426. Elsevier Ltd.

Hodgkison, R., M. Ayasse, C. Häberlein, S. Schulz, A. Zubaid, W. A. W. Mustapha, T. H. Kunz, and E. K. V. Kalko. 2013. Fruit bats and bat fruits: the evolution of fruit scent in relation to the foraging behaviour of bats in the New and Old World tropics. Funct. Ecol. 27:1075-1084.

Hodgkison, R., M. Ayasse, E. K. V. Kalko, C. Häberlein, S. Schulz, W. A. W. Mustapha, A. Zubaid, and T. H. Kunz. 2007. Chemical ecology of fruit bat foraging behavior in relation to the fruit odors of two species of Paleotropical bat-dispersed figs (Ficus hispida and Ficus scortechinii). J. Chem. Ecol. 33:2097-2110.

Hohmann, G. 2009. The diets of non-human primates: frugivory, food processing, and food sharing. Pp. 1-14 in The Evolution of Hominin Diets: Integrating Approaches to the Study of Palaeolithic Subsistence. Springer Science \& Business Media, Heidelberg.

Howe, H. F. 1980. Monkey dispersal and waste of a Neotropical fruit. Ecology 61:944-959.

Howe, H. F. 1986. Seed dispersal by fruit-eating birds and mammals. Pp. 123-189 in D. R. Murray, ed. Seed Dispersal. Academic Press, San-Diego.

Howe, H. F., and M. N. Miriti. 2004. When seed dispersal matters. Bioscience 54:651-660.

Howe, H. F., and J. Smallwood. 1982. Ecology of seed dispersal. Annu. Rev. Ecol. Syst. 13:201228.

Howe, H. F., and L. C. Westley. 1988. Ecological Relationships of Plants and Animals. Oxford University Press, New-York.

Howe, H. F., and L. C. Westley. 1986. Ecology of pollination and seed dispersal. Pp. 185-215 in M. J. Crawley, ed. Plant Ecology. Blackwell Scientific Publications, London.

Hübener, F., and M. Laska. 1998. Assessing olfactory performance in an Old World primate, Macaca nemestrina. Physiol. Behav. 64:521-527.

Hutcheon, J. M., J. A. W. Kirsch, and T. Garland Jr. 2002. A comparative analysis of brain size in relation to foraging ecology and phylogeny in the chiroptera. Brain. Behav. Evol. 60:165-180.

Irwin, M. T., F. J. Raharison, H. Rakotoarimanana, E. Razanadrakoto, E. Ranaivoson, J. Rakotofanala, and C. Randrianarimanana. 2007. Diademed sifakas (Propithecus diadema) use olfaction to forage for the inflorescences of subterranean parasitic plants (Balanophoraceae: Langsdorffia sp ., and Cytinaceae: Cytinus sp .). Int. J. Primatol. 69:471-476. 
Jacobs, G. H. 2009. Evolution of colour vision in mammals. Philos T Roy Soc B 364:2957-2967. Janson, C. H. 1983. Adaptation of fruit morphology to dispersal agents in a Neotropical forest. Science 219:187-189.

Janzen, D. H. 1970. Herbivores and the number of tree species in tropical forests. Am. Nat. 104:501-528.

Janzen, D. H. 1980. When is it coevolution? Evolution 34:611-612.

Jerison, H. J. 1955. Brain to body ratios and the evolution of intelligence. Science 121:447-449.

Jordano, P. 1995. Agiosperm fleshy fruits and seed dispersers: a comparative analysis of adaptation and constraints in plant-animal inetractions. Am. Nat. 145:163-191.

Julliot, C. 1996a. Fruit choice by red howler monkeys (Alouatta seniculus) in a tropical rain forest. Am. J. Primatol. 40:261-282.

Julliot, C. 1996b. Seed dispersal by red howling monkeys (Alouatta seniculus) in the tropical rain forest of French Guiana. Int. J. Primatol. 17:239-258.

Julliot, C., and D. Sabatier. 1993. Diet of the red howler monkey (Alouatta seniculus) in French Guiana. Int. J. Primatol. 14:527-550.

Kalko, E. K. V., and M. Ayasse. 2009. Study and analysis of odor involved in the behavioral ecology of bats. Pp. 491-499 in T. H. Kunz and S. Parsons, eds. Ecological and Behavioral Methods for the Study of Bats. The Johns Hopkins University Press, Baltimore.

Kant, M. R., P. M. Bleeker, M. van Wijk, R. C. Schuurink, and M. A. Haring. 2009. Plant volatiles in defence. Adv. Bot. Res. 51:613-666.

Kaplin, B. A., and J. E. Lambert. 2002. Effectiveness of seed dispersal by Cercopithecus monkeys: implications for seed input into degraded areas. Pp. 351-364 in D. J. Levey, W. R. Silva, and M. Galetti, eds. Frugivory and Seed Dispersal: Ecology, Evolution and Conservation. CABI, Oxon.

Kappel, P., S. Hohenbrink, and U. Radespiel. 2011. Experimental evidence for olfactory predator recognition in wild mouse lemurs. Am. J. Primatol. 73:928-938.

Kappeler, P. M., and E. W. Heymann. 1996. Nonconvergence in the evolution of primate life history and socio-ecology. Biol. J. Linn. Soc. 59:297-326.

Kirk, E. C., and R. F. Kay. 2004. The evolution of high visual acuity in the Anthropoidea. Pp. 539602 in C. F. Ross and R. F. Kay, eds. Anthropoid Origins: New Visions. Kluwer Academic / Plenum Publishers, New-York.

Knogge, C., and E. W. Heymann. 2003. Seed dispersal by sympatric tamarins, Saguinus mystax and Saguinus fuscicollis: diversity and characteristics of plant species. Folia Primatol. 74:33-47.

Knudsen, J. T., R. Eriksson, J. Gershenzon, and B. Ståhl. 2006. Diversity and distribution of floral scent. Bot. Rev. 72:1-120.

Korine, C., and E. K. V. Kalko. 2005. Fruit detection and discrimination by small fruit-eating bats (Phyllostomidae): echolocation call design and olfaction. Behav. Ecol. Sociobiol. 59:12-23.

Lambert, J. E. 2001. Red-tailed guenons (Cercopithecus ascanius) and Strychnos mitis: evidence for plant benefits beyond seed dispersal. Int. J. Primatol. 22:189-201.

Laska, M., V. Bauer, and L. T. Hernandez Salazar. 2007a. Self-anointing behavior in free-ranging spider monkeys (Ateles geoffroyi) in Mexico. Primates 48:160-163. 
Laska, M., R. M. R. Bautista, D. Höfelmann, V. Sterlemann, and L. T. Hernandez Salazar. 2007b. Olfactory sensitivity for putrefaction-associated thiols and indols in three species of non-human primate. J. Exp. Biol. 210:4169-4178.

Laska, M., P. Freist, and S. Krause. 2007c. Which senses play a role in nonhuman primate food selection? A comparison between squirrel monkeys and spider monkeys. Am. J. Primatol. 69:282-294.

Laska, M., and D. Freyer. 1997. Olfactory discrimination ability for aliphatic esters in squirrel monkeys and humans. Chem. Senses 22:457-65.

Laska, M., D. Genzel, and A. Wieser. 2005a. The number of functional olfactory receptor genes and the relative size of olfactory brain structures are poor predictors of olfactory discrimination performance with enantiomers. Chem. Senses 30:171-175.

Laska, M., L. T. Hernandez Salazar, and E. Rodriguez Luna. 2003. Successful acquisition of an olfactory discrimination paradigm by spider monkeys, Ateles geoffroyi. Physiol. Behav. 78:329329.

Laska, M., D. Höfelmann, D. Huber, and M. Schumacher. 2006a. The frequency of occurrence of acyclic monoterpene alcohols in the chemical environment does not determine olfactory sensitivity in nonhuman primates. J. Chem. Ecol. 32:1317-1331.

Laska, M., and R. Hudson. 1993. Discriminating parts from the whole: determinants of odor mixture perception in squirrel monkeys, Saimiri sciureus. J. Comp. Physiol. A 173:249-256.

Laska, M., R. M. Rivas Bautista, and L. T. Hernandez Salazar. 2006b. Olfactory sensitivity for aliphatic alcohols and aldehydes in spider monkeys (Ateles geoffroyi). Am. J. Phys. Anthropol. 129:112-120.

Laska, M., and A. Seibt. 2002a. Olfactory sensitivity for aliphatic alcohols in squirrel monkeys and pigtail macaques. J. Exp. Biol. 205:1633-1643.

Laska, M., and A. Seibt. 2002b. Olfactory sensitivity for aliphatic esters in squirrel monkeys and pigtail macaques. Behav. Brain Res. 134:165-174.

Laska, M., A. Seibt, and A. Weber. 2000. "Microsmatic" primates revisited: olfactory sensitivity in the squirrel monkey. Chem. Senses 25:47-53.

Laska, M., A. Wieser, and L. T. Hernandez Salazar. 2005b. Olfactory responsiveness to two odorous steroids in three species of nonhuman primates. Chem. Senses 30:505-511.

Le Gros Clark, W. E. 1971. The Antecedents of Man. Edinburgh University Press, Edinburgh.

Leishman, M. R., I. J. Wright, A. T. Moles, and M. Westoby. 2000. The evolutionary ecology of seed size. Pp. 31-58 in M. Fenner, ed. Seeds: the Ecology of Regeneration in Plant Communities. CABI, Oxon.

Leonhardt, S. D., J. Tung, J. B. Camden, M. Leal, and C. M. Drea. 2008. Seeing red: behavioral evidence of trichromatic color vision in strepsirrhine primates. Behav. Ecol. 20:1-12.

Lerdau, M., and H. L. Throop. 2000. Sources of variability in isoprene emission and photosynthesis in two species of tropical wet forest trees. Biotropica 32:670-676.

Link, A., and P. R. Stevenson. 2004. Fruit dispersal syndromes in animal disseminated plants at Tinigua National Park, Colombia. Rev. Chil. Hist. Nat. 77:319-334.

Lledo, P., G. Gheusi, and J. Vincent. 2005. Information processing in the mammalian olfactory system. Physiol. Rev. 85:281-317. 
Lomáscolo, S. B., D. J. Levey, R. T. Kimball, B. M. Bolker, and H. T. Alborn. 2010. Dispersers shape fruit diversity in Ficus (Moraceae). P Natl Acad Sci USA 107:14668-14672.

Lomáscolo, S. B., and H. M. Schaefer. 2010. Signal convergence in fruits: a result of selection by frugivores? J. Evol. Biol. 23:614-624.

Lomáscolo, S. B., P. Speranza, and R. T. Kimball. 2008. Correlated evolution of fig size and color supports the dispersal syndromes hypothesis. Oecologia 156:783-796.

Lord, J. M. 2002. Have frugivores influenced the evolution of fruit traits in New Zealand? Pp. 5568 in D. J. Levey, W. R. Silva, and M. Galetti, eds. Frugivory and Seed Dispersal: Ecology, Evolution, and Conservation. CABI, Wallingford.

Løtvedt, P. K., S. K. Murali, L. T. Hernandez Salazar, and M. Laska. 2012. Olfactory sensitivity for "green odors" (aliphatic C6 alcohols and C6 aldehydes) - a comparative study in male CD-1 mice (Mus musculus) and female spider monkeys (Ateles geoffroyi). Pharmacol. Biochem. Behav. 101:450-457.

Lunau, K. 1992. Innate recognition of flowers by bumble bees: orientation of antennae to visual stamen signals. Can. J. Zool. 70:2139-2144.

Mack, A. L. 2000. Did fleshy fruit pulp evolve as a defence against seed loss rather than as a dispersal mechanism? J. Biosci. 25:93-97.

Mainland, J., and N. Sobel. 2006. The sniff is part of the olfactory percept. Chem. Senses 31:181196.

Martin, J. P., M. Doucet, R. C. Knox, and D. J. Mennill. 2011. Body size correlates negatively with the frequency of distress calls and songs of Neotropical birds. J. F. Ornithol. 82:259-268.

Matsui, A., Y. Go, and Y. Niimura. 2010. Degeneration of olfactory receptor gene repertories in primates: no direct link to full trichromatic vision. Mol. Biol. Evol. 27:1192-200.

Matsumoto, Y., C. Hiramatsu, Y. Matsushita, N. Ozawa, R. Ashino, M. Nakata, S. Kasagi, A. Di Fiore, C. M. Schaffner, F. Aureli, A. D. Melin, and S. Kawamura. 2014. Evolutionary renovation of $\mathrm{L} / \mathrm{M}$ opsin polymorphism confers a fruit discrimination advantage to ateline New World monkeys. Mol. Ecol. 23:1799-1812.

Matsumoto-Oda, A., N. Kutsukake, K. Hosaka, and T. Matsusaka. 2007. Sniffing behaviors in Mahale chimpanzees. Primates 48:81-85.

McConkey, K. R., F. Aldy, A. Ario, and D. J. Chivers. 2002. Selection of fruit by gibbons (Hylobates muelleri $\times$ agilis) in the rain forests of central Borneo. Int. J. Primatol. 23:123-145.

McGarvey, D. J., and R. Croteau. 1995. Terpenoid metabolism. Plant Cell 7:1015-1026.

McGrath, M. J., and C. Karahadian. 1994. Evaluation of physical, chemical, and sensory properties of pawpaw fruit (Asimina triloba) as indicators of ripeness. J. Agric. Food Chem. 42:968-974.

McKey, D. 1975. The ecology of coevolved seed dispersal systems. Pp. 159-191 in L. E. Gilbert and P. H. Raven, eds. Co-evolution of animals and plants. University of Texas Press, Austin.

Melin, A. D., L. M. Fedigan, C. Hiramatsu, T. Hiwatashi, N. Parr, and S. Kawamura. 2009. Fig foraging by dichromatic and trichromatic Cebus capucinus in a tropical dry forest. Int. J. Primatol. 30:753-775.

Melin, A. D., C. Hiramatsu, N. A. Parr, Y. Matsushita, S. Kawamura, and L. M. Fedigan. 2014. The behavioral ecology of color vision: considering fruit conspicuity, detection distance and dietary 
importance. Int. J. Primatol. 35:258-287.

Michael, R. P., R. W. Bonsall, and D. Zumpe. 1976. Evidence for chemical communication in primates. Vitam. Horm. 34:137-186.

Mori, K., and H. Manabe. 2014. Unique characteristics of the olfactory system. Pp. 1-18 in K. Mori, ed. The Olfactory System: from Molecules to Motivational Behaviors. Springer, Tokyo.

Mori, K., H. Nagao, and Y. Yoshihara. 1999. The olfactory bulb: coding and processing of odor molecule information. Science 286:711-715.

Nathan, R., and H. C. Muller-Landau. 2000. Spatial patterns of seed dispersal, their determinants and consequences for recruitment. Trends Ecol. Evol. 15:278-285.

Nathan, R., F. M. Schurr, O. Spiegel, O. Steinitz, A. Trakhtenbrot, and A. Tsoar. 2008. Mechanisms of long-distance seed dispersal. Trends Ecol. Evol. 23:638-647.

Nei, M., Y. Niimura, and M. Nozawa. 2008. The evolution of animal chemosensory receptor gene repertoires: roles of chance and necessity. Nat. Rev. Genet. 9:951-963.

Nekaris, K. A. 2005. Foraging behaviour of the slender loris (Loris lydekkerianus lydekkerianus): implications for theories of primate origins. J. Hum. Evol. 49:289-300.

Neville, M. K., K. E. Glander, F. Braza, and A. B. Rylands. 1988. The howling monkeys. Pp. 349354 in R. A. Mittermeier, A. B. Rylands, A. F. Coimbra-Fliho, and G. A. B. de Fonesco, eds. Ecology and Behavior of Neotropical Primates. World Wildlife Fund, Washington, D.C.

Nevitt, G. A. 2000. Olfactory foraging by Antarctic procellariiform seabirds: life at high Reynolds numbers. Biol. Bull. 198:245-253.

Nevo, O., R. O. Garri, L. T. Hernandez Salazar, S. Schulz, E. W. Heymann, M. Ayasse, and M. Laska. 2015. Chemical recognition of fruit ripeness in spider monkeys (Ateles geoffroyi). Sci. Rep. 5:14895. Nature Publishing Group.

Nevo, O., and E. W. Heymann. 2015. Led by the Nose: Olfaction in Primate Feeding Ecology. Evol. Anthropol. 24:137-148.

Niimura, Y. 2012. Evolution of chemosensory receptor genes in primates and other mammals. Pp. 43-62 in H. Hirai, Y. Go, and H. Imai, eds. Post-genome Biology of Primates. Springer, Tokyo.

Nuñez-Iturri, G., and H. F. Howe. 2007. Bushmeat and the fate of trees with seeds dispersed by large primates in a lowland rain forest in western Amazonia. Biotropica 39:348-354.

Nuñez-Iturri, G., O. Olsson, and H. F. Howe. 2008. Hunting reduces recruitment of primatedispersed trees in Amazonian Peru. Biol. Conserv. 141:1536-1546.

Oliveira, A. C. M., and S. F. Ferrari. 2000. Seed dispersal by black-handed tamarins, Saguinus midas niger (Callitrichinae, Primates): implications for the regeneration of degraded forest habitats in eastern Amazonia. J. Trop. Ecol. 16:709-716.

Orme, D., R. P. Freckleton, G. Thomas, T. Petzoldt, S. Fritz, N. Isaac, and W. Pearse. 2012. Caper: comparative analyses of phylogenetics and evolution in R. R package version 0.5.

Pablo-Rodríguez, M., L. T. Hernández-Salazar, F. Aureli, and C. M. Schaffner. 2015. The role of sucrose and sensory systems in fruit selection and consumption of Ateles geoffroyi in Yucatan, Mexico. J. Trop. Ecol. 1-7.

Palagi, E., and L. Dapporto. 2006. Beyond odor discrimination: demonstrating individual recognition by scent in Lemur catta. Chem. Senses 31:437-443. 
Palminteri, S., G. V. Powell, and C. A. Peres. 2012. Advantages of granivory in seasonal environments: feeding ecology of an arboreal seed predator in Amazonian forests. Oikos 121:1896-1904.

Peres, C. A. 1994. Diet and feeding ecology of gray woolly monkeys (Lagothrix lagotricha cana) in Central Amazonia: comparisons with other atelines. Int. J. Primatol. 15:333-372.

Pfrommer, A. 2009. Seed dispersal ecology of Leonia cymosa (Violaceae) in the rain forest of Eastern Ecuador. Bayrische Julius-Maximilians Universität Würzburg.

Phillips, K. A., L. M. S. Goodchild, M. E. Haas, M. J. Ulyan, and S. Petro. 2004. Use of visual, acoustic, and olfactory information during embedded invertebrate foraging in brown capuchins (Cebus apella). J. Comp. Psychol. 118:200-205.

Piep, M., U. Radespiel, E. Zimmermann, S. Schmidt, and B. M. Siemers. 2008. The sensory basis of prey detection in captive-born grey mouse lemurs, Microcebus murinus. Anim. Behav. 75:871878.

Porter, J., B. Craven, R. M. Khan, S.-J. Chang, I. Kang, B. Judkewitz, J. Volpe, G. Settles, and N. Sobel. 2007. Mechanisms of scent-tracking in humans. Nat. Neurosci. 10:27-29.

Poulsen, J. R., C. J. Clark, E. F. Connor, and T. B. Smith. 2002. Differential resource use by primates and hornbills: implications for seed dispersal. Ecology 83:228-240.

Quinn, G. P., and M. J. Keough. 2002. Experimental Design and Data Analysis for Biologists. Cambridge University Press, Cambridge.

R Core Team. 2014. R: A language and environment for statistical computing. R Foundation for Statistical Computing, Vienna, Austria. URL http://www.R-project.org/.

Regan, B. C., C. Julliot, B. Simmen, F. Viénot, P. Charles-Dominique, and J. D. Mollon. 2001. Fruits, foliage and the evolution of primate colour vision. Philos T Roy Soc B 356:229-283.

Reinehr, A. 2010. Seed-dispersal of Leonia cymosa (Violaceae) by the sympatric tamarins Saguinus mystax and Saguinus fuscicollis, Primates, Callitrichidae. MSc Thesis. Universität Hohemheim.

Renner, S. S. 1989. A survey of reproductive biology in Neotropical Melastomataceae and Memecylaceae. Ann. Missouri Bot. Gard. 76:496-518.

Ribeiro, P. F. M., P. R. Manger, K. C. Catania, J. H. Kaas, and S. Herculano-Houzel. 2014. Greater addition of neurons to the olfactory bulb than to the cerebral cortex of eulipotyphlans but not rodents, afrotherians or primates. Front. Neuroanat. 8:23.

Rizvanovic, A., M. Amundin, and M. Laska. 2013. Olfactory discrimination ability of Asian elephants (Elephas maximus) for structurally related odorants. Chem. Senses 38:107-118.

Rodríguez, A., B. Alquézar, and L. Peña. 2013. Fruit aromas in mature fleshy fruits as signals of readiness for predation and seed dispersal. New Phytol. 197:36-48.

Rodríguez, A., V. San Andrés, M. Cervera, A. Redondo, B. Alquézar, T. Shimada, J. Gadea, M. J. Rodrigo, L. Zacarías, L. Palou, M. M. López, P. Castañera, and L. Peña. 2011. Terpene downregulation in orange reveals the role of fruit aromas in mediating interactions with insect herbivores and pathogens. Plant Physiol. 156:793-802.

Rouquier, S., A. Blancher, and D. Giorgi. 2000. The olfactory receptor gene repertoire in primates and mouse: evidence for reduction of the functional fraction in primates. P Natl Acad Sci USA 97:2870-2874. 
Rushmore, J., S. D. Leonhardt, and C. M. Drea. 2012. Sight or scent: lemur sensory reliance in detecting food quality varies with feeding ecology. PLoS One 7:e41558.

Sarrafchi, A., A. M. E. Odhammer, L. T. Hernandez Salazar, and M. Laska. 2013. Olfactory sensitivity for six predator odorants in CD-1 mice, human subjects, and spider monkeys. PLoS One 8:e80621.

Schaefer, H. M., and G. D. Ruxton. 2011. Animal-plant communication. Oxford University Press, Oxford.

Schaefer, H. M., A. Valido, and P. Jordano. 2014. Birds see the true colours of fruits to live off the fat of the land. Proc. R. Soc. B Biol. Sci. 281:20132516.

Schiestl, F. P. 2015. Ecology and evolution of floral volatile- mediated information transfer in plants. New Phytol. 206:571-577.

Schupp, E. W. 1993. Quantity, quality and the effectiveness of seed dispersal by animals. Vegetatio 107/108:15-29.

Schupp, E. W., P. Jordano, and J. M. Gómez. 2010. Seed dispersal effectiveness revisited: a conceptual review. New Phytol. 188:333-353.

Siemers, B. M. 2013. The sensory ecology of foraging for animal prey. Pp. 257-263 in J. Masters, M. Gamba, and F. Génin, eds. Leaping Ahead: Advances in Prosimian Biology. Springer, NewYork.

Siemers, B. M., H. R. Goerlitz, E. Robsomanitrandrasana, M. Piep, J.-B. Ramanamanjato, D. Rakotondravony, O. Ramilijaona, and J. U. Ganzhorn. 2007. Sensory basis of food detection in wild Microcebus murinus. Int. J. Primatol. 28:291-304.

Smith, A. C. 1997. Comparative ecology of saddleback (Saguinus fuscicollis) and moustached (Saguinus mystax) tamarins. University of Reading.

Smith, R. J., and W. L. Jungers. 1997. Body mass in comparative primatology. J. Hum. Evol. 32:523-559.

Smith, T. D., and K. P. Bhatnagar. 2004. Microsmatic primates: reconsidering how and when size matters. Anat. Rec. B. New Anat. 279:24-31.

Smith, T. D., K. P. Bhatnagar, P. Tuladhar, and A. M. Burrows. 2004. Distribution of olfactory epithelium in the primate nasal cavity: are microsmia and macrosmia valid morphological concepts? Anat. Rec. Part A 281:1173-1181.

Smith, T. D., J. B. Rossie, and K. P. Bhatnagar. 2007. Evolution of the nose and nasal skeleton in primates. Evol. Anthropol. 16:132-146.

Snow, D. W. 1981. Tropical frugivorous birds and their food plants: a world survey. Biotropica 13:1-14.

Springer, M. S., R. W. Meredith, J. Gatesy, C. a Emerling, J. Park, D. L. Rabosky, T. Stadler, C. Steiner, O. a Ryder, J. E. Janečka, C. a Fisher, and W. J. Murphy. 2012. Macroevolutionary dynamics and historical biogeography of primate diversification inferred from a species supermatrix. PLoS One 7:e49521.

Steiger, S. S., A. E. Fidler, M. Valcu, and B. Kempenaers. 2008. Avian olfactory receptor gene repertoires: evidence for a well-developed sense of smell in birds? Proc. R. Soc. London. Ser. B Biol. Sci. 275:2309-2317.

Stephan, H., H. D. Frahm, and G. Baron. 1981. New and revised data on volumes of brain 
structures in insectivores and primates. Folia Primatol. 35:1-29.

Stevenson, P. R. 2000. Seed dispersal by woolly monkeys (Lagothrix lagothricha) at Tinigua National Park, Colombia: dispersal distance, germination rates, and dispersal quantity. Am. J. Primatol. 50:275-89.

Stevenson, P. R., M. J. Quiiiones, and J. A. Ahumada. 2000. Influence of fruit availability on ecological overlap among four Neotropical primates at Tinigua National Park, Colombia. Biotropica 32:533-544.

Stiles, F. G., and L. Rosselli. 1993. Consumption of fruits of the Melastomataceae by birds: how diffuse is coevolution? Pp. 57-73 in T. H. Fleming and A. Estrada, eds. Frugivory and Seed Dispersal: Ecological and Evolutionary Aspects. Kluwer Academic Publishers, Dodrecht.

Sündermann, D., M. Scheumann, and E. Zimmermann. 2008. Olfactory predator recognition in predator-naïve gray mouse lemurs (Microcebus murinus). J. Comp. Psychol. 122:146-155.

Sussman, R. W. 1991. Primate origins and the evolution of angiosperms. Am. J. Primatol. 223:209-223.

Sussman, R. W., D. T. Rasmussen, and P. H. Raven. 2013. Rethinking primate origins again. Am. J. Primatol. 75:95-106.

Terborgh, J. W. 1992. Diversity and the tropical rain forest. Scientific American Library, New-York.

Tewksbury, J. J., and G. P. Nabhan. 2001. Directed deterrence by capsaicin in chilies. Nature 412:403-404.

Thies, W., E. K. V. Kalko, and H.-U. Schnitzler. 1998. The roles of echolocation and olfaction in two Neotropical fruit-eating bats, Carollia perspicillata and C. castanea, feeding on Piper. Behav. Ecol. Sociobiol. 42:397-409.

Thomas, D. W. 1984. Fruit intake and energy budgets of frugivorous bats. Physiol. Zool. 57:457467.

Tiffney, B. H. 1986. Evolution of seed dispersal syndromes according to the fossil record. Pp. 273-305 in D. R. Murray, ed. Seed Dispersal. Academic Press, San-Diego.

Tiffney, B. H. 1984. Seed size, dispersal syndromes, and the rise of the angiosperms: evidence and hypothesis. Ann. Missouri Bot. Gard. 71:551-576.

Tiffney, B. H. 2004. Vertebrate dispersal of seed plants through time. Annu. Rev. Ecol. Evol. Syst. 35:1-29.

Tiffney, B. H., and S. J. Mazer. 1995. Angiosperm growth habit, dispersal and diversification reconsidered. Evol. Ecol. 9:93-117.

Torigoe, T. 1985. Comparison of object manipulation among 74 species of non-human primates. Primates 26:182-194.

Touhara, K. 2014. Odor and pheromone molecules, receptors and behavioral responses. Pp. 1938 in K. Mori, ed. The Olfactory System: from Molecules to Motivational Behaviors. Springer Japan, Tokyo.

Traveset, A. 1998. Effect of seed passage through vertebrate frugivores' guts on germination: a review. Perspect. Plant Ecol. Evol. Syst. 1:151-190.

Traveset, A., and M. Verdú. 2002. A meta-analysis of the effect of gut treatment on seed germination. Pp. 339-350 in D. J. Levey, W. R. Silva, and M. Galetti, eds. Seed Dispersal and Frugivory: Ecology, Evolution and Conservation. CABI, Oxon. 
Ueno, Y. 1994. Olfactory discrimination of eight food flavors in the capuchin monkey (Cebus apella): comparison between fruity fishy odors. Primates 35:301-310.

Valenta, K., R. J. Burke, S. A. Styler, D. A. Jackson, A. D. Melin, and S. M. Lehman. 2013. Colour and odour drive fruit selection and seed dispersal by mouse lemurs. Sci. Rep. 3:2424.

Valido, A., H. M. Schaefer, and P. Jordano. 2011. Colour, design and reward: phenotypic integration of fleshy fruit displays. J. Evol. Biol. 24:751-760.

van der Pijl, L. 1982. Principles of Dispersal in Higher Plants. 3rd ed. Springer, Berlin.

van Roosmalen, M. G. M. 1985a. Fruits of the Guiana Flora. Institute of Systematic Botany, Utrecht.

van Roosmalen, M. G. M. 1985b. Habitat preference, diet, feeding strategy and social organization of the black spider monkey (Ateles paniscus paniscus Linnaeus 1758) in Surinam. Acta Amaz. 15:1-238.

Vander Wall, S. B., and W. S. Longland. 2004. Diplochory: are two seed dispersers better than one? Trends Ecol. Evol. 19:155-61.

Venables, W. N., and B. D. Ripley. 2002. Modern applied statistics with S. Fourth Edition. Springer, New-York.

Verhagen, J. V, D. W. Wesson, T. I. Netoff, J. A. White, and M. Wachowiak. 2007. Sniffing controls an adaptive filter of sensory input to the olfactory bulb. Nat. Neurosci. 10:631-639.

Vickers, N. J. 2000. Mechanisms of animal navigation in odor plumes. Biol. Bull. 198:203-212.

Visalberghi, E., and E. Addessi. 2000. Response to changes in food palatability in tufted capuchin monkeys, Cebus apella. Anim. Behav. 59:231-238.

Vogel, E. R., M. Neitz, and N. J. Dominy. 2006. Effect of color vision phenotype on the foraging of wild white-faced capuchins, Cebus capucinus. Behav. Ecol. 18:292-297.

Voigt, F. A., B. Bleher, J. Fietz, J. U. Ganzhorn, D. Schwab, and K. Böhning-Gaese. 2004. A comparison of morphological and chemical fruit traits between two sites with different frugivore assemblages. Oecologia 141:94-104.

Wahaj, S. a, D. J. Levey, A. K. Sanders, and M. L. Cipollini. 1998. Control of gut retention time by secondary metabolites in ripe Solanum fruits. Ecology 79:2309-2319.

Wallén, H., I. Engström, L. T. Hernandez Salazar, and M. Laska. 2012. Olfactory sensitivity for six amino acids: a comparative study in CD-1 mice and spider monkeys. Amino Acids 42:1475-1485.

Weiss, K. M. 2014. I smell a rat! (and 999,999,999,999 other things, too). Evol. Anthropol. 23:166171.

Wenny, D. G. 2001. Advantages of seed dispersal: a re-evaluation of directed dispersal. Evol. Ecol. Res. 3:51-74.

Wheelwright, N. T. 1993. Fruit size in a tropical tree species: variation, preference by birds, and heritability. Pp. 163-174 in T. H. Fleming and A. Estrada, eds. Frugivory and Seed Dispersal: Ecological and Evolutionary Aspects. Kluwer Academic Publishers, Dordrecht.

Wheelwright, N. T., W. A. Haber, K. G. Murray, and C. Guindon. 1984. Tropical fruit-eating birds and their food plants: a survey of a Costa Rican lower montane forest. Biotropica 16:173-192.

Whitehead, S. R., and M. D. Bowers. 2013. Evidence for the adaptive significance of secondary compounds in vertebrate-dispersed fruits. Am. Nat. 182:563-577. 
Wrangham, R. W. 1980. An ecological model of female-bonded primate groups. Behaviour 75:262-300.

Wright, P. C. 1989. The nocturnal primate niche in the New World. J. Hum. Evol. 18:635-658.

Wysocki, C. J., and G. Preti. 2004. Facts, fallacies, fears, and frustrations with human pheromones. Anat. Rec. Part A 281:1201-1211.

Yamagiwa, J., and A. K. Basabose. 2009. Fallback foods and dietary partitioning among Pan and Gorilla. Am. J. Phys. Anthropol. 140:739-750.

Young, J. M., and B. J. Trask. 2002. The sense of smell: genomics of vertebrate odorant receptors. Hum. Mol. Genet. 11:1153-60.

Zahavi, A. 1975. Mate selection-a selection for a handicap. J. Theor. Biol. 53:205-14.

Zahavi, A., and A. Zahavi. 1997. The Handicap Principle. Oxford University Press, New-York.

Zhang, X., O. De la Cruz, J. M. Pinto, D. Nicolae, S. Firestein, and Y. Gilad. 2007. Characterizing the expression of the human olfactory receptor gene family using a novel DNA microarray. Genome Biol. 8:R86.

Zschoke, A., and R. Thomsen. 2014. Sniffing behaviours in guenons. Folia Primatol. 85:244-251. 
Appendix 1 - supplementary materials for chapter 3

\section{APPENDIX 1 - SUPPLEMENTARY MATERIALS FOR CHAPTER 3}

\section{Methods and materials}

Model System. Odor samples of fruits were collected at the Estación Biológica Quebrada Blanco, Loreto, north-eastern Peru ( $4^{\circ} 21^{\prime} S 73^{\circ} 09^{\prime} \mathrm{W}$ ), between February and June 2013 (for more details on the site, see Heymann 1995). Due to the difficulties of obtaining a sufficient amount of samples in the tropics, we restricted our analyses to four model species. Model species were selected during field work because it is impossible to predict which species would provide fruits in sufficient amounts in a given year. We selected species that were abundant and dyssynchronous (i.e. provided both ripe and unripe fruits at the same time). This was important for both technical and theoretical reasons: first, to allow sampling in both modes under the same conditions and second, because in fully synchronous species signals for ripeness are probably less important since there is no need to distinguish ripe from unripe fruits. We selected species that allowed to control for phylogeny and that are either primate or bird dispersed. Couma macrocarpa (Apocynaceae) is a canopy tree whose fruits are yellow, globular and large $(>4 \mathrm{~cm}$ in diameter) and dispersed by many primate species (Peres 1994; Culot 2009). Leonia cymosa (Violaceae) is an understory tree producing yellow globular fruits (ca. $3 \mathrm{~cm}$ in diameter) dispersed exclusively by primates (Pfrommer 2009; Reinehr 2010). Fruits of both species are protected with a thick husk preventing access from small birds. Psychotria cincta (Rubiaceae) provides small (ca. $0.5 \mathrm{~cm}$ in diameter) soft orange fruits. Fruits of this genus are dispersed by small birds (Snow 1981; Gorchov et al. 1995). Maieta guianensis (Melastomataceae) provides small (ca. $1 \mathrm{~cm}$ in diameter) dark and soft berries like most other fleshy-fruit producing Melastomataceae. Apart from a single genus (Bellucia), all fleshy fruits from this family are dispersed by birds (Renner 1989; Stiles and Rosselli 1993). Both species were observed to be consumed by small understory passerines (O.N., pers. obs.) and are usually inaccessible to primates because they grow on small bushes or treelets. Long-term behavioral and ecological field work on tamarin monkeys (Saguinus mystax, Saguinus nigrifrons), which are smaller than most other Neotropical primates, confirmed that $P$. cincta and M. guianensis are never consumed by primates even when they do have physical access to the 
Appendix 1 - supplementary materials for chapter 3

fruits (Smith 1997; Knogge and Heymann 2003; Culot 2009). Finally, all four model species are from families that include species dispersed by the other dispersal vector (i.e. primates in the case of the two bird-dispersed species and vice versa) (Wheelwright et al. 1984; Julliot and Sabatier 1993; Ballard and Sytsma 2000,Culot et al. 2010b) as well as species producing nonendozoochoric dry fruits (Bremer and Eriksson 1992; Clausing et al. 2000; Bolmgren and Eriksson 2005; Ballard et al. 2014). Thus, while we cannot provide any information regarding ancestral states, fruit traits in these families showed, over evolutionary time, malleability that allowed many lineages to adapt in response to selection pressures exerted by different dispersal vectors. Each primate-dispersed species is phylogenetically closer to one bird-dispersed species than they are to one another (Davies et al. 2004).

Collection of Fruit Odor Samples. Fresh ripe and unripe fruits were collected from different trees/shrubs, cleaned with a wet cloth and sampled, each fruit individually, within up to $4 \mathrm{~h}$ of removal. $P$. cincta infructescences often contain small clusters of 2-3 ripe or unripe fruits, which means that if it signals ripeness via the olfactory channel, it may be a combined signal of more than one fruit. We thus always sampled two fruits from the same infructescense at a time from this species. Within species, sampling in the four conditions (ripe/unripe, intact/open) was unordered, although for natural reasons ripe fruits (intact/open) tended to be sampled later in the season. For sampling of the pulp odor (open fruits), fruits were cut through with a clean knife. Fruits were enclosed in chambers of $30 \mathrm{~cm}$ unused inert oven bags (Toppits, Germany) for $2.5 \mathrm{~h}$. Then, their headspace odors were collected for $10 \mathrm{~min}$ in a constant airflow of $330 \mathrm{ml} / \mathrm{min}$ onto a self-made adsorbent trap containing $3 \mathrm{mg}$ 1:1 mixture of Tenax-TA and Carbotrap (both Supelco, Sigma-Aldrich, Germany) (Dötterl et al. 2005). Adsorbent traps were installed on a cleaned (ethanol EMSURE 99.9\% and pentane EMSURE 99\%, Merck, Germany) Teflon tube that penetrated the chamber and was the only opening in the system. Adsorbent traps were then packed individually in clean $1.5 \mathrm{ml}$ vials sealed with Teflon caps (Supelco, Sigma-Aldrich, Germany) and held frozen in $-20^{\circ} \mathrm{C}$ until analysis apart from a 4-day transportation period in which they were packed isolated with freezing packs (Techni-ice, Australia) frozen to $-30^{\circ} \mathrm{C}$. Sample size 
Appendix 1 - supplementary materials for chapter 3

was 9-15 fruits per species and condition (ripe or unripe, intact or open), totaling at $\mathrm{N}=40-49$ samples per species in all conditions. To identify possible contaminations, we took at least one control sample (same conditions, chamber without a fruit) on every sampling day.

Chemical Analyses. Samples were analyzed on a Hewlett Packard HP 6890 Series gas chromatographic-mass selective detector (GC-MS; Agilent Quadrupol 5972) equipped with a DB5ms capillary column (30 m long, $250 \mu \mathrm{m}$ in diameter, film thickness: $0.25 \mu \mathrm{m}$, J\&W) which had been fitted with the ChromatoProbe kit (Dötterl and Jürgens 2005). An adsorbent trap was loaded into the probe, which was then inserted into the modified GC injector at an injector temperature of $300^{\circ} \mathrm{C}$. Volatile traps were injected splitless at an oven temperature of $40^{\circ} \mathrm{C}$. After $1 \mathrm{~min}$ the split valve was opened and the oven temperature increased by $6^{\circ} \mathrm{C} / \mathrm{min}$ until reaching $120^{\circ} \mathrm{C}$ and then by $10^{\circ} \mathrm{C} / \mathrm{min}$ until reaching $310^{\circ} \mathrm{C}$, a temperature which was held for 6 extra min. Tentative identification of most compounds was carried out using the Adams (2007) mass spectral data bases on MSD Chemstation (build 75) and confirmed by comparison of their retention indices with those from the published libraries (Adams 2007; NIST 11). Retention indices were calculated using an external alkane standard (C9 - C31) which was ran at the same conditions several times. We confirmed the identity of the majority of dominant compounds (see Tab. $\underline{8.1}, \underline{8.2}, \underline{8.3}, \underline{8.4}$ ) by running known synthetic compounds at the same conditions. Compounds were quantified using Amdis 2.71. Absolute amounts were estimated using an external standard of $0.01 \mathrm{mg} p$-Cymene which we ran 10 times under identical conditions.

Statistical Analysis. Before further analyses, in each species, we excluded compounds which were present in less than eight samples of that species and compounds considered as contamination. We considered a compound to be a contaminant when it was present in similar concentrations in the control samples. Few compounds (e.g. limonene) were contaminants but also appeared in much bigger concentrations in C. macrocarpa, which implied that it was a genuine peak in this 
Appendix 1 - supplementary materials for chapter 3

species. We thus excluded it from the species in which the concentration was on par with that of control samples, but kept it for C. macrocarpa.

To compare the odor profiles of ripe and unripe fruits in either intact or open conditions, we first, for each species separately, applied a principal component analysis $(P C A)$ to reduce the number of variables and avoid collinearity between them. Since C. macrocarpa showed a high number of minor compounds, in order to avoid inflating the number of variables in the statistical tests we only used compounds whose absolute amounts exceeded $100 \mathrm{ng}$ in at least one sample. We then used the PCs that accounted for at least $90 \%$ of the original variance (C. macrocarpa: 8 PCs, $L$. cymosa: 7, P. cincta: 5, M. guianensis: 4) as new variables in discriminant function analyses (DFA) applied for each species separately. We used DFAs to graphically examine, for each species, whether odor profiles of ripe and unripe fruits in both conditions (intact and open) form separate clusters. To answer the biologically relevant question whether odor is indicative of the ripeness level of the fruits and quantify the level of discrimination between the clusters of ripe and unripe fruits (intact or open) identified in the DFAs, we applied a post-hoc analysis comprised of a series of 8 pair-wise MANOVA tests. MANOVA tests are mathematically identical to DFA (Quinn and Keough 2002) and thus allow using the same data for this analysis in which, for each species, we tested whether the odor profiles of ripe and unripe intact fruits are significantly different from one another, and similarly whether profiles of open ripe and unripe fruits are different. $P$-values were then subjected to the Bonferroni correction for multiple testing. All analyses were conducted on $R$ 3.0.3 (R Core Team 2014) with packages MASS (Venables and Ripley 2002) and ade4 (Dray and Dufour 2007) and on SPSS v. 22. 


\section{Additional results}

Tab. 8.1. Compounds identified from Couma macrocarpa. Mean ( \pm SD) estimated absolute amounts (mg) of compounds in all Couma macrocarpa samples in $\mathrm{mg}$ after $2.5 \mathrm{~h}$ sampling and retention indices (RI). Absolute amounts were calculated based on an external standard. Values are rounded and thus very small amounts are sometimes presented as 0 . Compounds presented are those present in at least 8 samples in a species, including the minor compounds ( $<100 \mathrm{ng}$ in at least one sample) that were considered in the odor richness analysis but not odor production, PCA and DFA (see Methods). ID method: methods used for identification of the respective compound. RI: retention index matching published values. MS: strong match with published mass spectra. REF: compound identification verified by running a synthetic compound or, in cases of enantiomers and close derivatives, a mixture of known compounds. In unknown compounds, ID method provides dominant MS ions. Compound classes: terpenoids (TRP), aromatic compounds (AR), aldehydes (ALD), alcohols (ALC), esters (EST), acids (AC).

\begin{tabular}{|c|c|c|c|c|c|c|c|c|}
\hline Code & ID & Class & RI & $\begin{array}{l}\text { ID } \\
\text { method }\end{array}$ & $\begin{array}{l}\text { Ripe } \\
\text { intact }\end{array}$ & $\begin{array}{l}\text { Unripe } \\
\text { intact }\end{array}$ & $\begin{array}{l}\text { Ripe } \\
\text { open }\end{array}$ & $\begin{array}{l}\text { Unripe } \\
\text { open }\end{array}$ \\
\hline C1 & Cumene & AR & 9.23 & $\begin{array}{l}\text { RI, MS, } \\
\text { REF }\end{array}$ & $\begin{array}{r}0.004 \\
\pm(0.007)\end{array}$ & $\begin{array}{r}0.003 \\
\pm(0.007)\end{array}$ & $\begin{array}{r}0.01 \\
\pm(0.027)\end{array}$ & $\begin{array}{r}0.009 \\
\pm(0.029)\end{array}$ \\
\hline C2 & $\alpha$-Thujene & TRP & 9.25 & $\mathrm{RI}, \mathrm{MS}$ & $\begin{array}{r}0.001 \\
\pm(0.002)\end{array}$ & $\begin{array}{r}0.003 \\
\pm(0.007)\end{array}$ & $\begin{array}{r}0.001 \\
\pm(0.004)\end{array}$ & $\begin{array}{r}0.025 \\
\pm(0.061)\end{array}$ \\
\hline C3 & $\alpha$-Pinene & TRP & 9.32 & $\mathrm{RI}, \mathrm{MS}$ & $\begin{array}{r}0.051 \\
\pm(0.068)\end{array}$ & $\begin{array}{r}0.028 \\
\pm(0.044)\end{array}$ & $\begin{array}{r}0.094 \\
\pm(0.19)\end{array}$ & $\begin{array}{r}0.119 \\
\pm(0.276)\end{array}$ \\
\hline C4 & Benzaldehyde & $\begin{array}{l}\text { AR, } \\
\text { ALD }\end{array}$ & 9.63 & $\begin{array}{l}\text { RI, MS, } \\
\text { REF }\end{array}$ & $\begin{array}{r}0.01 \\
\pm(0.011)\end{array}$ & $\begin{array}{r}0.001 \\
\pm(0.003)\end{array}$ & $\begin{array}{r}0.189 \\
\pm(0.441)\end{array}$ & $\begin{array}{r}0.011 \\
\pm(0.02)\end{array}$ \\
\hline C5 & Sabinene & TRP & 9.73 & $\mathrm{RI}, \mathrm{MS}$ & $\begin{array}{r}0.001 \\
\pm(0.003)\end{array}$ & $\begin{array}{r}0.027 \\
\pm(0.046)\end{array}$ & $\begin{array}{r}0 \\
\pm(0.001)\end{array}$ & $\begin{array}{r}0.03 \\
\pm(0.04)\end{array}$ \\
\hline C6 & Myrcene & TRP & 9.89 & $\begin{array}{l}\text { RI, MS, } \\
\text { REF }\end{array}$ & $\begin{array}{r}0.023 \\
\pm(0.021)\end{array}$ & $\begin{array}{l}0.063 \\
\pm(0.1)\end{array}$ & $\begin{array}{r}0.047 \\
\pm(0.042)\end{array}$ & $\begin{array}{r}0.068 \\
\pm(0.084)\end{array}$ \\
\hline C7 & $\alpha$-Terpinene & TRP & 10.17 & $\mathrm{RI}, \mathrm{MS}$ & $0 \pm(0)$ & $\begin{array}{r}0.008 \\
\pm(0.014)\end{array}$ & $\begin{array}{r}0 \\
\pm(0.001)\end{array}$ & $\begin{array}{r}0.022 \\
\pm(0.041)\end{array}$ \\
\hline C8 & $p$-Cymene & $\begin{array}{l}\text { AR, } \\
\text { TRP }\end{array}$ & 10.25 & $\begin{array}{l}\text { RI, MS, } \\
\text { REF }\end{array}$ & $\begin{array}{r}0.004 \\
\pm(0.005)\end{array}$ & $\begin{array}{r}0.029 \\
\pm(0.038)\end{array}$ & $\begin{array}{r}0.011 \\
\pm(0.008)\end{array}$ & $\begin{array}{r}0.094 \\
\pm(0.161)\end{array}$ \\
\hline C9 & Limonene & TRP & 10.30 & $\begin{array}{l}\text { RI, MS, } \\
\text { REF }\end{array}$ & $\begin{array}{r}0.026 \\
\pm(0.019)\end{array}$ & $\begin{array}{r}0.274 \\
\pm(0.418)\end{array}$ & $\begin{array}{r}0.057 \\
\pm(0.051)\end{array}$ & $\begin{array}{r}0.258 \\
\pm(0.339)\end{array}$ \\
\hline C10 & $\begin{array}{l}(E)- \\
\beta-\text { Ocimene }\end{array}$ & TRP & 10.47 & $\begin{array}{l}\text { RI, MS, } \\
\text { REF }\end{array}$ & $\begin{array}{r}0.067 \\
\pm(0.105)\end{array}$ & $\begin{array}{r}0.014 \\
\pm(0.015)\end{array}$ & $\begin{array}{r}0.116 \\
\pm(0.208)\end{array}$ & $\begin{array}{r}0.025 \\
\pm(0.031)\end{array}$ \\
\hline C11 & $\gamma$-Terpinene & TRP & 10.58 & $\begin{array}{l}\text { RI, MS, } \\
\text { REF }\end{array}$ & $\begin{array}{r}0.008 \\
\pm(0.025)\end{array}$ & $\begin{array}{r}0.018 \\
\pm(0.029)\end{array}$ & $\begin{array}{r}0.001 \\
\pm(0.002)\end{array}$ & $\begin{array}{r}0.028 \\
\pm(0.052)\end{array}$ \\
\hline C12 & Acetophenone & AR & 10.67 & $\begin{array}{l}\text { RI, MS, } \\
\text { REF }\end{array}$ & $\begin{array}{r}0.002 \\
\pm(0.003)\end{array}$ & $\begin{array}{r}0.001 \\
\pm(0.002)\end{array}$ & $\begin{array}{r}0.003 \\
\pm(0.004)\end{array}$ & $\begin{array}{r}0.001 \\
\pm(0.003)\end{array}$ \\
\hline C13 & $\begin{array}{l}\text { cis-Linalool } \\
\text { oxide } \\
\text { (furanoid) }\end{array}$ & $\begin{array}{l}\text { TRP, } \\
\text { ALC }\end{array}$ & 10.71 & $\mathrm{RI}, \mathrm{MS}$ & $\begin{array}{r}0.002 \\
\pm(0.004)\end{array}$ & $0 \pm(0)$ & $\begin{array}{r}0.016 \\
\pm(0.042)\end{array}$ & $\begin{array}{r}0.012 \\
\pm(0.014)\end{array}$ \\
\hline
\end{tabular}


Appendix 1 - supplementary materials for chapter 3

\begin{tabular}{|c|c|c|c|c|c|c|c|c|}
\hline C14 & Unknown & & 10.74 & $\begin{array}{l}\text { Dominant } \\
\text { ions: } 43 \text {, } \\
85,69,58\end{array}$ & $\begin{array}{r}0.003 \\
\pm(0.005)\end{array}$ & $\begin{array}{r}0.017 \\
\pm(0.039)\end{array}$ & $\begin{array}{r}0.006 \\
\pm(0.006)\end{array}$ & $\begin{array}{r}0.011 \\
\pm(0.026)\end{array}$ \\
\hline C15 & $\begin{array}{l}\text { Unknown } \\
\text { monoterpene }\end{array}$ & TRP & 10.86 & $\begin{array}{l}\text { Dominant } \\
\text { ions: } \\
91, \\
91,79, \\
105,77, \\
41, \quad 93, \\
136,119\end{array}$ & $0 \pm(0)$ & $\begin{array}{r}0.003 \\
\pm(0.006)\end{array}$ & $\begin{array}{r}0.001 \\
\pm(0.003)\end{array}$ & $\begin{array}{r}0.01 \\
\pm(0.018)\end{array}$ \\
\hline C16 & $\begin{array}{l}\text { trans-Linalool } \\
\text { oxide } \\
\text { (furanoid) }\end{array}$ & $\begin{array}{l}\text { TRP, } \\
\text { ALC }\end{array}$ & 10.87 & $\mathrm{RI}, \mathrm{MS}$ & $\begin{array}{r}0.001 \\
\pm(0.004)\end{array}$ & $0 \pm(0)$ & $\begin{array}{r}0.016 \\
\pm(0.042)\end{array}$ & $\begin{array}{r}0.009 \\
\pm(0.011)\end{array}$ \\
\hline C17 & $p$-Cymenene & $\begin{array}{l}\text { AR, } \\
\text { TRP }\end{array}$ & 10.91 & $\begin{array}{l}\text { RI, MS, } \\
\text { REF }\end{array}$ & $0 \pm(0)$ & $\begin{array}{r}0.005 \\
\pm(0.007)\end{array}$ & $0 \pm(0)$ & $\begin{array}{r}0.015 \\
\pm(0.028)\end{array}$ \\
\hline C18 & Unknown & & 10.93 & $\begin{array}{l}\text { Dominant } \\
\text { ions: } 89, \\
41\end{array}$ & $\begin{array}{r}0.001 \\
\pm(0.002)\end{array}$ & $\begin{array}{r}0.003 \\
\pm(0.006)\end{array}$ & $\begin{array}{r}0.003 \\
\pm(0.007)\end{array}$ & $\begin{array}{r}0.015 \\
\pm(0.02)\end{array}$ \\
\hline C19 & $\begin{array}{l}\text { Methyl } \\
\text { benzoate }\end{array}$ & $\begin{array}{l}\text { AR, } \\
\text { EST }\end{array}$ & 10.96 & $\mathrm{RI}, \mathrm{MS}$ & $\begin{array}{r}0.001 \\
\pm(0.003)\end{array}$ & $0 \pm(0)$ & $\begin{array}{r}0.009 \\
\pm(0.012)\end{array}$ & $0 \pm(0)$ \\
\hline C20 & Unknown & & 10.98 & $\begin{array}{l}\text { Dominant } \\
\text { ions: } 55 \text {, } \\
41, \quad 69, \\
84,96\end{array}$ & $0 \pm(0)$ & $0 \pm(0)$ & $\begin{array}{r}0.022 \\
\pm(0.023)\end{array}$ & $0 \pm(0)$ \\
\hline C21 & Linalool & $\begin{array}{l}\text { TRP, } \\
\text { ALC }\end{array}$ & 11.01 & $\begin{array}{l}\text { RI, MS, } \\
\text { REF }\end{array}$ & $\begin{array}{r}0.014 \\
\pm(0.027)\end{array}$ & $\begin{array}{r}0.002 \\
\pm(0.007)\end{array}$ & $\begin{array}{r}0.065 \\
\pm(0.158)\end{array}$ & $\begin{array}{r}0.077 \\
\pm(0.111)\end{array}$ \\
\hline C22 & (Z)-6-Nonenal & ALD & 11.03 & $\mathrm{RI}, \mathrm{MS}$ & $0 \pm(0)$ & $0 \pm(0)$ & $\begin{array}{r}0.033 \\
\pm(0.039)\end{array}$ & $0 \pm(0)$ \\
\hline C23 & Nonanal & ALD & 11.06 & $\begin{array}{l}\text { RI, MS, } \\
\text { REF }\end{array}$ & $\begin{array}{r}0.02 \\
\pm(0.014)\end{array}$ & $\begin{array}{r}0.015 \\
\pm(0.012)\end{array}$ & $\begin{array}{r}0.106 \\
\pm(0.065)\end{array}$ & $\begin{array}{r}0.013 \\
\pm(0.013)\end{array}$ \\
\hline C24 & $\begin{array}{l}(E)-4,8- \\
\text { Dimethyl- } \\
1,3,7- \\
\text { nonatriene }\end{array}$ & TRP & 11.14 & $\mathrm{RI}, \mathrm{MS}$ & $\begin{array}{r}0.01 \\
\pm(0.014)\end{array}$ & $\begin{array}{r}0.024 \\
\pm(0.042)\end{array}$ & $\begin{array}{r}0.054 \\
\pm(0.107)\end{array}$ & $\begin{array}{r}0.152 \\
\pm(0.185)\end{array}$ \\
\hline C25 & allo-Ocimene & TRP & 11.29 & $\begin{array}{l}\text { RI, MS, } \\
\text { REF }\end{array}$ & $\begin{array}{r}0.011 \\
\pm(0.014)\end{array}$ & $\begin{array}{r}0.003 \\
\pm(0.006)\end{array}$ & $\begin{array}{r}0.019 \\
\pm(0.025)\end{array}$ & $\begin{array}{r}0.01 \\
\pm(0.013)\end{array}$ \\
\hline C26 & $\begin{array}{l}\text { (2E,6Z)-2,6- } \\
\text { Nonadienal }\end{array}$ & ALD & 11.53 & $\mathrm{RI}, \mathrm{MS}$ & $\begin{array}{r}0.002 \\
\pm(0.004)\end{array}$ & $0 \pm(0)$ & $\begin{array}{r}0.077 \\
\pm(0.073)\end{array}$ & $\begin{array}{r}0.001 \\
\pm(0.002)\end{array}$ \\
\hline C27 & $\begin{array}{l}\text { Trans-2- } \\
\text { nonenal }\end{array}$ & ALD & 11.61 & $\begin{array}{l}\text { RI, MS, } \\
\text { REF }\end{array}$ & $\begin{array}{r}0.008 \\
\pm(0.014)\end{array}$ & $0 \pm(0)$ & $\begin{array}{r}0.213 \\
\pm(0.169)\end{array}$ & $\begin{array}{r}0.002 \\
\pm(0.007)\end{array}$ \\
\hline C28 & $\begin{array}{l}\text { Methyl } \\
\text { salicylate }\end{array}$ & AR & 11.93 & $\begin{array}{l}\text { RI, MS, } \\
\text { REF }\end{array}$ & $\begin{array}{r}0.034 \\
\pm(0.057)\end{array}$ & $\begin{array}{r}0 \\
\pm(0.001)\end{array}$ & $\begin{array}{r}0.269 \\
\pm(0.506)\end{array}$ & $\begin{array}{r}0.011 \\
\pm(0.013)\end{array}$ \\
\hline C2 & $\begin{array}{l}\text { Methyl 6- } \\
\text { nonenoate }\end{array}$ & EST & 12.21 & $\mathrm{RI}, \mathrm{MS}$ & $\begin{array}{r}0.01 \\
\pm(0.025)\end{array}$ & $0 \pm(0)$ & $\begin{array}{r}0.02 \\
\pm(0.01)\end{array}$ & $0 \pm(0)$ \\
\hline
\end{tabular}


Appendix 1 - supplementary materials for chapter 3

\begin{tabular}{|c|c|c|c|c|c|c|c|c|}
\hline C30 & $\begin{array}{l}\text { Methyl } \\
\text { nonanoate }\end{array}$ & EST & 12.25 & $\mathrm{RI}, \mathrm{MS}$ & $\begin{array}{r}0.003 \\
\pm(0.009)\end{array}$ & $0 \pm(0)$ & $\begin{array}{r}0.01 \\
\pm(0.006)\end{array}$ & $0 \pm(0)$ \\
\hline C31 & Unknown & & 12.29 & $\begin{array}{l}\text { Dominant } \\
\text { ions: } 123 \text {, } \\
43,180\end{array}$ & $\begin{array}{r}0 \\
\pm(0.001)\end{array}$ & $\begin{array}{r}0.003 \\
\pm(0.008)\end{array}$ & $\begin{array}{r}0 \\
\pm(0.001)\end{array}$ & $\begin{array}{r}0.002 \\
\pm(0.006)\end{array}$ \\
\hline C32 & Unknown & & 12.31 & $\begin{array}{l}\text { Dominant } \\
\text { ions: } 93, \\
43,121, \\
41, \quad 40, \\
121,108, \\
\begin{array}{lr}136, & 55, \\
79, & 66, \\
151, & 166\end{array}\end{array}$ & $0 \pm(0)$ & $0 \pm(0)$ & $\begin{array}{r}0.006 \\
\pm(0.011)\end{array}$ & $\begin{array}{r}0.001 \\
\pm(0.001)\end{array}$ \\
\hline C33 & $\begin{array}{l}\text { Ethyl } \\
\text { salicylate }\end{array}$ & AR & 12.71 & $\begin{array}{l}\text { RI, MS, } \\
\text { REF }\end{array}$ & $\begin{array}{r}0.015 \\
\pm(0.029)\end{array}$ & $0 \pm(0)$ & $\begin{array}{r}0.079 \\
\pm(0.089)\end{array}$ & $0 \pm(0)$ \\
\hline C34 & Neryl formate & TRP & 12.83 & $\mathrm{RI}, \mathrm{MS}$ & $\begin{array}{r}0.002 \\
\pm(0.004)\end{array}$ & $0 \pm(0)$ & $\begin{array}{r}0.013 \\
\pm(0.011)\end{array}$ & $0 \pm(0)$ \\
\hline C35 & $\alpha$-Cubebene & TRP & 13.50 & $\mathrm{RI}, \mathrm{MS}$ & $\begin{array}{r}0.123 \\
\pm(0.129)\end{array}$ & $\begin{array}{r}0.122 \\
\pm(0.152)\end{array}$ & $\begin{array}{r}0.116 \\
\pm(0.112)\end{array}$ & $\begin{array}{r}0.111 \\
\pm(0.126)\end{array}$ \\
\hline C36 & $\alpha$-Ylangene & TRP & 13.73 & $\mathrm{RI}, \mathrm{MS}$ & $\begin{array}{r}0.004 \\
\pm(0.007)\end{array}$ & $\begin{array}{r}0.006 \\
\pm(0.011)\end{array}$ & $\begin{array}{r}0.007 \\
\pm(0.01)\end{array}$ & $\begin{array}{r}0.012 \\
\pm(0.017)\end{array}$ \\
\hline C37 & $\alpha$-Copaene & TRP & 13.80 & $\begin{array}{l}\text { RI, MS, } \\
\text { REF }\end{array}$ & $\begin{array}{r}0.68 \\
\pm(0.459)\end{array}$ & $\begin{array}{r}0.74 \\
\pm(0.764)\end{array}$ & $\begin{array}{r}0.776 \\
\pm(0.757)\end{array}$ & $\begin{array}{r}1.21 \\
\pm(1.19)\end{array}$ \\
\hline C38 & $\beta$-Bourbonene & TRP & 13.87 & $\mathrm{RI}, \mathrm{MS}$ & $\begin{array}{r}0.003 \\
\pm(0.003)\end{array}$ & $\begin{array}{r}0.002 \\
\pm(0.003)\end{array}$ & $\begin{array}{r}0.003 \\
\pm(0.003)\end{array}$ & $\begin{array}{r}0.001 \\
\pm(0.001)\end{array}$ \\
\hline C39 & $\beta$-Cubebene & TRP & 13.90 & $\mathrm{RI}, \mathrm{MS}$ & $\begin{array}{r}0.013 \\
\pm(0.013)\end{array}$ & $\begin{array}{r}0.01 \\
\pm(0.014)\end{array}$ & $\begin{array}{r}0.01 \\
\pm(0.009)\end{array}$ & $\begin{array}{r}0.022 \\
\pm(0.026)\end{array}$ \\
\hline C40 & $\beta$-Elemene & TRP & 13.91 & $\mathrm{RI}, \mathrm{MS}$ & $\begin{array}{r}0.012 \\
\pm(0.019)\end{array}$ & $\begin{array}{r}0.014 \\
\pm(0.025)\end{array}$ & $\begin{array}{r}0.015 \\
\pm(0.023)\end{array}$ & $\begin{array}{r}0.001 \\
\pm(0.004)\end{array}$ \\
\hline & & & & $\begin{array}{l}\text { Dominant } \\
\text { ions: } 189 \text {, }\end{array}$ & & & & \\
\hline C41 & Unknown & & 14.00 & $\begin{array}{l}57, \quad 43, \\
133,91, \\
107,71, \\
148,204\end{array}$ & $\begin{array}{r}0.002 \\
\pm(0.006)\end{array}$ & $\begin{array}{r}0.004 \\
\pm(0.01)\end{array}$ & $\begin{array}{r}0.005 \\
\pm(0.008)\end{array}$ & $\begin{array}{r}0.004 \\
\pm(0.007)\end{array}$ \\
\hline C42 & $\begin{array}{l}(Z)- \\
\text { Caryophyllene }\end{array}$ & TRP & 14.07 & $\begin{array}{l}\text { RI, MS, } \\
\text { REF }\end{array}$ & $\begin{array}{r}0.007 \\
\pm(0.007)\end{array}$ & $\begin{array}{r}0.001 \\
\pm(0.002)\end{array}$ & $\begin{array}{r}0.025 \\
\pm(0.018)\end{array}$ & $\begin{array}{r}0.003 \\
\pm(0.01)\end{array}$ \\
\hline C43 & $\alpha$-Funebrene & TRP & 14.14 & $\begin{array}{l}\text { RI, MS, } \\
\text { REF }\end{array}$ & $\begin{array}{r}0.001 \\
\pm(0.003)\end{array}$ & $\begin{array}{r}0.004 \\
\pm(0.007)\end{array}$ & $\begin{array}{r}0.001 \\
\pm(0.004)\end{array}$ & $\begin{array}{r}0.001 \\
\pm(0.002)\end{array}$ \\
\hline C44 & $\begin{array}{l}(E)- \\
\text { Caryophyllene }\end{array}$ & TRP & 14.24 & $\begin{array}{l}\text { RI, MS, } \\
\text { REF }\end{array}$ & $\begin{array}{r}0.235 \\
\pm(0.189)\end{array}$ & $\begin{array}{r}0.073 \\
\pm(0.091)\end{array}$ & $\begin{array}{r}0.69 \\
\pm(0.452)\end{array}$ & $\begin{array}{r}0.057 \\
\pm(0.054)\end{array}$ \\
\hline C45 & $\beta$-Copaene & TRP & 14.34 & $\mathrm{RI}, \mathrm{MS}$ & $\begin{array}{r}0.005 \\
\pm(0.003)\end{array}$ & $\begin{array}{r}0.007 \\
\pm(0.008)\end{array}$ & $\begin{array}{r}0.007 \\
\pm(0.008)\end{array}$ & $\begin{array}{r}0.015 \\
\pm(0.02)\end{array}$ \\
\hline
\end{tabular}


Appendix 1 - supplementary materials for chapter 3

\begin{tabular}{|c|c|c|c|c|c|c|c|c|}
\hline C46 & $\begin{array}{l}\alpha- \\
\text { Bergamotene }\end{array}$ & TRP & 14.35 & $\mathrm{RI}, \mathrm{MS}$ & $\begin{array}{r}0.001 \\
\pm(0.003)\end{array}$ & $\begin{array}{r}0.009 \\
\pm(0.014)\end{array}$ & $\begin{array}{r}0 \\
\pm(0.001)\end{array}$ & $\begin{array}{r}0.002 \\
\pm(0.007)\end{array}$ \\
\hline C47 & $\begin{array}{l}\text { Unknown } \\
\text { sesquiterpene }\end{array}$ & TRP & 14.54 & RI & $\begin{array}{r}0.003 \\
\pm(0.004)\end{array}$ & $\begin{array}{r}0.008 \\
\pm(0.011)\end{array}$ & $\begin{array}{r}0.011 \\
\pm(0.015)\end{array}$ & $\begin{array}{r}0.008 \\
\pm(0.009)\end{array}$ \\
\hline C48 & $\begin{array}{l}\text { Unknown } \\
\text { sesquiterpene }\end{array}$ & TRP & 14.59 & MS & $\begin{array}{r}0.006 \\
\pm(0.015)\end{array}$ & $\begin{array}{r}0.01 \\
\pm(0.025)\end{array}$ & $\begin{array}{r}0.014 \\
\pm(0.026)\end{array}$ & $\begin{array}{r}0.008 \\
\pm(0.015)\end{array}$ \\
\hline C49 & $\alpha$-Humulene & TRP & 14.61 & $\begin{array}{l}\text { RI, MS, } \\
\text { REF }\end{array}$ & $\begin{array}{r}0.021 \\
\pm(0.017)\end{array}$ & $\begin{array}{r}0.012 \\
\pm(0.016)\end{array}$ & $\begin{array}{r}0.069 \\
\pm(0.039)\end{array}$ & $\begin{array}{r}0.007 \\
\pm(0.008)\end{array}$ \\
\hline C50 & $\gamma$-Muurolene & TRP & 14.78 & $\mathrm{RI}, \mathrm{MS}$ & $\begin{array}{r}0.003 \\
\pm(0.003)\end{array}$ & $\begin{array}{r}0.003 \\
\pm(0.004)\end{array}$ & $\begin{array}{r}0.006 \\
\pm(0.009)\end{array}$ & $\begin{array}{r}0.011 \\
\pm(0.024)\end{array}$ \\
\hline C51 & Unknown & & 14.86 & $\begin{array}{l}\text { Dominant } \\
\text { ions: } 41, \\
105,91, \\
69,162, \\
119,159\end{array}$ & $\begin{array}{r}0.001 \\
\pm(0.003)\end{array}$ & $\begin{array}{r}0.009 \\
\pm(0.018)\end{array}$ & $\begin{array}{r}0.001 \\
\pm(0.002)\end{array}$ & $\begin{array}{r}0.006 \\
\pm(0.01)\end{array}$ \\
\hline C52 & $\beta$-Selinene & TRP & 14.94 & $\mathrm{RI}, \mathrm{MS}$ & $\begin{array}{r}0.077 \\
\pm(0.078)\end{array}$ & $\begin{array}{r}0.101 \\
\pm(0.134)\end{array}$ & $\begin{array}{r}0.073 \\
\pm(0.054)\end{array}$ & $\begin{array}{r}0.037 \\
\pm(0.035)\end{array}$ \\
\hline C53 & $\alpha$-Selinene & TRP & 15.00 & $\begin{array}{l}\text { RI, MS, } \\
\text { REF }\end{array}$ & $\begin{array}{r}0.033 \\
\pm(0.033)\end{array}$ & $\begin{array}{r}0.04 \\
\pm(0.052)\end{array}$ & $\begin{array}{r}0.032 \\
\pm(0.027)\end{array}$ & $\begin{array}{r}0.02 \\
\pm(0.023)\end{array}$ \\
\hline C54 & $\begin{array}{l}(E, E)-\alpha- \\
\text { Farnesene }\end{array}$ & TRP & 15.05 & $\begin{array}{l}\text { RI, MS, } \\
\text { REF }\end{array}$ & $\begin{array}{r}0.032 \\
\pm(0.07)\end{array}$ & $\begin{array}{r}0.194 \\
\pm(0.363)\end{array}$ & $\begin{array}{r}0.035 \\
\pm(0.073)\end{array}$ & $\begin{array}{r}0.141 \\
\pm(0.258)\end{array}$ \\
\hline C55 & $\delta$-Amorphene & TRP & 15.22 & $\mathrm{RI}, \mathrm{MS}$ & $\begin{array}{r}0.012 \\
\pm(0.012)\end{array}$ & $\begin{array}{r}0.012 \\
\pm(0.016)\end{array}$ & $\begin{array}{r}0.018 \\
\pm(0.013)\end{array}$ & $\begin{array}{r}0.021 \\
\pm(0.024)\end{array}$ \\
\hline C56 & $\begin{array}{l}\text { cis- } \\
\text { Calamenene }\end{array}$ & TRP & 15.26 & $\mathrm{RI}, \mathrm{MS}$ & $\begin{array}{r}0.007 \\
\pm(0.01)\end{array}$ & $\begin{array}{r}0.003 \\
\pm(0.004)\end{array}$ & $\begin{array}{r}0.007 \\
\pm(0.009)\end{array}$ & $\begin{array}{r}0.013 \\
\pm(0.028)\end{array}$ \\
\hline C57 & $\begin{array}{l}\text { trans-Cadina- } \\
\text { 1,4-diene }\end{array}$ & TRP & 15.38 & $\mathrm{RI}, \mathrm{MS}$ & $\begin{array}{r}0.003 \\
\pm(0.005)\end{array}$ & $\begin{array}{r}0.004 \\
\pm(0.006)\end{array}$ & $\begin{array}{r}0.004 \\
\pm(0.005)\end{array}$ & $\begin{array}{r}0.004 \\
\pm(0.006)\end{array}$ \\
\hline C58 & $\alpha$-Calacorene & $\begin{array}{l}\text { TRP, } \\
\text { AR }\end{array}$ & 15.48 & $\mathrm{RI}, \mathrm{MS}$ & $\begin{array}{r}0.001 \\
\pm(0.002)\end{array}$ & $\begin{array}{r}0.001 \\
\pm(0.001)\end{array}$ & $\begin{array}{r}0.002 \\
\pm(0.003)\end{array}$ & $\begin{array}{r}0.005 \\
\pm(0.011)\end{array}$ \\
\hline C59 & $\begin{array}{l}\text { Unknown } \\
\text { sesquiterpene }\end{array}$ & TRP & 15.89 & RI & $0 \pm(0)$ & $0 \pm(0)$ & $\begin{array}{r}0.021 \\
\pm(0.02)\end{array}$ & $0 \pm(0)$ \\
\hline & TOTAL & & & & $\begin{array}{r}1.45 \\
\pm(0.86)\end{array}$ & $\begin{array}{r}1.77 \\
\pm(1.82)\end{array}$ & $\begin{array}{r}3.08 \\
\pm(1.29)\end{array}$ & $\begin{array}{r}2.41 \\
\pm(2.21)\end{array}$ \\
\hline
\end{tabular}


Appendix 1 - supplementary materials for chapter 3

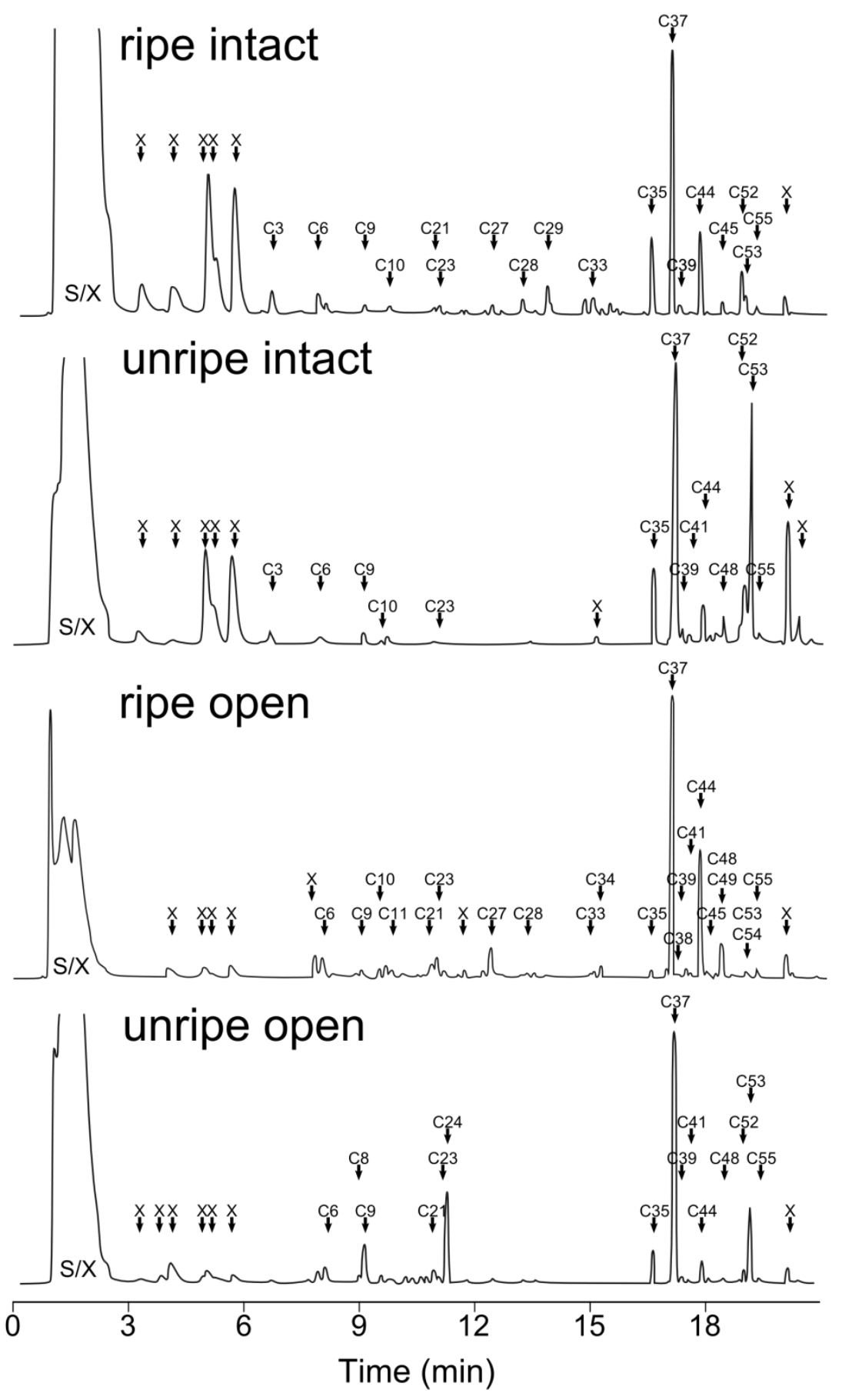

Fig. 8.1. Representative gas chromatograms of odor profiles of $\boldsymbol{C}$. macrocarpa. Compound names are shown in Tab. 8.1. X marks contaminants. SX marks a solvent \& unknown contaminant peaks present in all samples, including control. Presented samples were chosen according to their proximity to the group means (e.g. ripe intact). However, they may markedly deviate from the group mean with regards to some individual compounds and are thus for illustration only. 
Tab. 8.2. Compound identified from Leonia cymosa. Mean ( \pm SD) estimated absolute amounts $(\mathrm{mg})$ of compounds in all Leonia cymosa samples in $\mathrm{mg}$ after $2.5 \mathrm{~h}$ sampling and retention indices (RI). Absolute amounts were calculated based on an external standard. Values are rounded and thus very small amounts are sometimes presented as 0 . Compounds presented are those present in at least 8 samples in a species (see Methods). ID method: methods used for identification of the respective compound. RI: retention index matching published values. MS: strong match with published mass spectra. REF: compound identification verified by running a synthetic compound or, in cases of enantiomers and close derivatives, a mixture of known compounds. In unknown compounds, ID method provides dominant MS ions.

\begin{tabular}{|c|c|c|c|c|c|c|c|c|c|}
\hline Code & ID & Class & RI & $\begin{array}{l}\text { ID } \\
\text { metho }\end{array}$ & & $\begin{array}{l}\text { Ripe } \\
\text { intact }\end{array}$ & $\begin{array}{l}\text { Unripe } \\
\text { intact }\end{array}$ & $\begin{array}{l}\text { Ripe } \\
\text { open }\end{array}$ & $\begin{array}{l}\text { Unripe } \\
\text { open }\end{array}$ \\
\hline L1 & Cumene & $A R$ & 9.23 & $\begin{array}{l}\mathrm{Rl}, \quad \\
\mathrm{REF}\end{array}$ & MS, & $\begin{array}{r}0.001 \\
\pm(0.002)\end{array}$ & $\begin{array}{r}0.013 \\
\pm(0.023)\end{array}$ & $\begin{array}{r}0.011 \\
\pm(0.034)\end{array}$ & $\begin{array}{r}0.018 \\
\pm(0.054)\end{array}$ \\
\hline L2 & Benzaldehyde & $\begin{array}{l}A R \\
A L D\end{array}$ & 9.63 & $\begin{array}{l}\text { RI, } \\
\text { REF }\end{array}$ & MS, & $\begin{array}{r}0.014 \\
\pm(0.019)\end{array}$ & $\begin{array}{r}0.004 \\
\pm(0.009)\end{array}$ & $\begin{array}{r}0.062 \\
\pm(0.153)\end{array}$ & $\begin{array}{r}0.05 \\
\pm(0.153)\end{array}$ \\
\hline L3 & Myrcene & TRP & 9.89 & $\begin{array}{l}\mathrm{RI}, \quad \\
\mathrm{REF}\end{array}$ & MS, & $0 \pm(0)$ & $0 \pm(0)$ & $\begin{array}{r}0.025 \\
\pm(0.037)\end{array}$ & $\begin{array}{r}0.008 \\
\pm(0.028)\end{array}$ \\
\hline L4 & $\begin{array}{l}\text { Unknown } \\
\text { monoterpene }\end{array}$ & TRP & 10.06 & $\mathrm{RI}, \mathrm{MS}$ & & $0 \pm(0)$ & $0 \pm(0)$ & $\begin{array}{r}1.083 \\
\pm(1.302)\end{array}$ & $0 \pm(0)$ \\
\hline L5 & p-Cymene & $\begin{array}{l}\text { AR, } \\
\text { TRP }\end{array}$ & 10.25 & $\begin{array}{l}\mathrm{RI}, \quad \\
\mathrm{REF}\end{array}$ & MS, & $\begin{array}{r}0.003 \\
\pm(0.004)\end{array}$ & $\begin{array}{r}0.001 \\
\pm(0.001)\end{array}$ & $\begin{array}{r}0.02 \\
\pm(0.043)\end{array}$ & $\begin{array}{r}0.009 \\
\pm(0.032)\end{array}$ \\
\hline L6 & $\begin{array}{l}(Z)- \\
\beta-\text { Ocimene }\end{array}$ & TRP & 10.36 & $\begin{array}{l}\mathrm{RI}, \quad \\
\mathrm{REF}\end{array}$ & MS, & $0 \pm(0)$ & $0 \pm(0)$ & $\begin{array}{r}0.043 \\
\pm(0.059)\end{array}$ & $\begin{array}{r}0.003 \\
\pm(0.011)\end{array}$ \\
\hline L7 & $\begin{array}{l}(E)- \\
\beta \text {-Ocimene }\end{array}$ & TRP & 10.49 & $\begin{array}{l}\mathrm{Rl}, \quad \\
\mathrm{REF}\end{array}$ & MS, & $\begin{array}{r}0.006 \\
\pm(0.008)\end{array}$ & $0 \pm(0)$ & $\begin{array}{r}5.999 \\
\pm(7.201)\end{array}$ & $\begin{array}{r}0.009 \\
\pm(0.01)\end{array}$ \\
\hline L8 & Acetophenone & $A R$ & 10.67 & $\begin{array}{l}\mathrm{RI}, \quad \\
\mathrm{REF}\end{array}$ & MS, & $\begin{array}{r}0.006 \\
\pm(0.006)\end{array}$ & $\begin{array}{r}0.008 \\
\pm(0.01)\end{array}$ & $\begin{array}{r}0.009 \\
\pm(0.009)\end{array}$ & $\begin{array}{r}0.003 \\
\pm(0.004)\end{array}$ \\
\hline L9 & $\begin{array}{l}2,6 \text {-Dimethyl- } \\
1,3,5,7- \\
\text { octatetraene }\end{array}$ & TRP & 10.81 & $\mathrm{RI}, \mathrm{MS}$ & & $\begin{array}{r}0.001 \\
\pm(0.002)\end{array}$ & $\begin{array}{r}0.002 \\
\pm(0.004)\end{array}$ & $\begin{array}{r}0.091 \\
\pm(0.091)\end{array}$ & $\begin{array}{r}0.001 \\
\pm(0.003)\end{array}$ \\
\hline L10 & Unknown & & 10.87 & $\begin{array}{l}\text { Domin } \\
\text { ions: } \\
105, \\
119\end{array}$ & $\begin{array}{l}\text { nant } \\
91 \\
77\end{array}$ & $0 \pm(0)$ & $0 \pm(0)$ & $\begin{array}{r}0.031 \\
\pm(0.038)\end{array}$ & $0 \pm(0)$ \\
\hline L11 & $p$-Cymenene & $A R$ & 10.92 & $\begin{array}{l}\mathrm{RI}, \quad \\
\mathrm{REF}\end{array}$ & MS, & $0 \pm(0)$ & $0 \pm(0)$ & $\begin{array}{r}0.199 \\
\pm(0.207)\end{array}$ & $\begin{array}{r}0.001 \\
\pm(0.002)\end{array}$ \\
\hline L12 & $\begin{array}{l}p-1,3,8- \\
\text { Menthatriene }\end{array}$ & TRP & 11.18 & $\mathrm{RI}, \mathrm{MS}$ & & $0 \pm(0)$ & $0 \pm(0)$ & $\begin{array}{r}0.171 \\
\pm(0.234)\end{array}$ & $0 \pm(0)$ \\
\hline L13 & allo-Ocimene & TRP & 11.29 & $\begin{array}{l}\mathrm{RI}, \quad \\
\mathrm{REF}\end{array}$ & MS, & $\begin{array}{r}0.001 \\
\pm(0.003)\end{array}$ & $0 \pm(0)$ & $\begin{array}{r}0.37 \\
\pm(0.62)\end{array}$ & $\begin{array}{r}0.012 \\
\pm(0.028)\end{array}$ \\
\hline L14 & $\begin{array}{l}\text { neo-allo- } \\
\text { Ocimene }\end{array}$ & TRP & 11.41 & $\begin{array}{l}\mathrm{Rl}, \quad \\
\mathrm{REF}\end{array}$ & MS, & $0 \pm(0)$ & $0 \pm(0)$ & $\begin{array}{r}0.133 \\
\pm(0.187)\end{array}$ & $\begin{array}{r}0.001 \\
\pm(0.002)\end{array}$ \\
\hline
\end{tabular}


Appendix 1 - supplementary materials for chapter 3

\begin{tabular}{|c|c|c|c|c|c|c|c|c|}
\hline L15 & $\begin{array}{l}\text { (Z)-3-hexenyl } \\
\text { 2- } \\
\text { methylbutyrate }\end{array}$ & EST & 12.32 & $\mathrm{RI}, \mathrm{MS}$ & $0 \pm(0)$ & $0 \pm(0)$ & $\begin{array}{r}0.002 \\
\pm(0.004)\end{array}$ & $\begin{array}{r}0.016 \\
\pm(0.025)\end{array}$ \\
\hline L16 & $\alpha$-Cubebene & TRP & 13.50 & $\mathrm{RI}, \mathrm{MS}$ & $\begin{array}{r}0.01 \\
\pm(0.025)\end{array}$ & $\begin{array}{r}0.002 \\
\pm(0.007)\end{array}$ & $\begin{array}{r}0.009 \\
\pm(0.016)\end{array}$ & $\begin{array}{r}0.031 \\
\pm(0.033)\end{array}$ \\
\hline L17 & $\alpha$-Ylangene & TRP & 13.73 & $\mathrm{RI}, \mathrm{MS}$ & $\begin{array}{r}0.008 \\
\pm(0.024)\end{array}$ & $\begin{array}{r}0.001 \\
\pm(0.002)\end{array}$ & $\begin{array}{r}0.012 \\
\pm(0.017)\end{array}$ & $\begin{array}{r}0.036 \\
\pm(0.081)\end{array}$ \\
\hline L18 & $\alpha$-Copaene & TRP & 13.79 & $\begin{array}{l}\mathrm{RI}, \quad \mathrm{MS}, \\
\mathrm{REF}\end{array}$ & $\begin{array}{r}0.043 \\
\pm(0.106)\end{array}$ & $\begin{array}{r}0.007 \\
\pm(0.009)\end{array}$ & $\begin{array}{r}0.06 \\
\pm(0.085)\end{array}$ & $\begin{array}{r}0.237 \\
\pm(0.535)\end{array}$ \\
\hline L19 & $\begin{array}{l}\text { Unknown } \\
\text { sesquiterpene }\end{array}$ & TRP & 13.90 & $\mathrm{RI}, \mathrm{MS}$ & $\begin{array}{r}0.002 \\
\pm(0.008)\end{array}$ & $\begin{array}{r}0 \\
\pm(0.002)\end{array}$ & $\begin{array}{r}0.005 \\
\pm(0.009)\end{array}$ & $\begin{array}{r}0.013 \\
\pm(0.02)\end{array}$ \\
\hline L20 & $\alpha$-Funebrene & TRP & 14.13 & $\begin{array}{l}\text { RI, MS, } \\
\text { REF }\end{array}$ & $\begin{array}{r}0 \\
\pm(0.001)\end{array}$ & $\begin{array}{r}0.004 \\
\pm(0.006)\end{array}$ & $\begin{array}{r}0.003 \\
\pm(0.005)\end{array}$ & $\begin{array}{r}0.005 \\
\pm(0.01)\end{array}$ \\
\hline L21 & $\begin{array}{l}\beta \text {-Copaene } \\
<\text { beta-> }\end{array}$ & TRP & 14.34 & $\mathrm{RI}, \mathrm{MS}$ & $\begin{array}{r}0.004 \\
\pm(0.009)\end{array}$ & $0 \pm(0)$ & $\begin{array}{r}0.008 \\
\pm(0.012)\end{array}$ & $\begin{array}{r}0.02 \\
\pm(0.052)\end{array}$ \\
\hline L22 & $\gamma$-Muurolene & TRP & 14.78 & $\mathrm{RI}, \mathrm{MS}$ & $\begin{array}{r}0.001 \\
\pm(0.004)\end{array}$ & $\begin{array}{r}0.001 \\
\pm(0.003)\end{array}$ & $\begin{array}{r}0.003 \\
\pm(0.004)\end{array}$ & $\begin{array}{r}0.007 \\
\pm(0.02)\end{array}$ \\
\hline L23 & $\alpha$-Muurolene & TRP & 15.01 & $\mathrm{RI}, \mathrm{MS}$ & $\begin{array}{r}0.004 \\
\pm(0.014)\end{array}$ & $\begin{array}{r}0 \\
\pm(0.002)\end{array}$ & $\begin{array}{r}0.017 \\
\pm(0.018)\end{array}$ & $\begin{array}{r}0.027 \\
\pm(0.059)\end{array}$ \\
\hline L24 & $\delta$-Amorphene & TRP & 15.21 & $\mathrm{RI}, \mathrm{MS}$ & $\begin{array}{r}0.003 \\
\pm(0.007)\end{array}$ & $\begin{array}{r}0.001 \\
\pm(0.002)\end{array}$ & $\begin{array}{r}0.013 \\
\pm(0.014)\end{array}$ & $\begin{array}{r}0.017 \\
\pm(0.022)\end{array}$ \\
\hline L25 & $\begin{array}{l}\text { cis- } \\
\text { Calamenene }\end{array}$ & TRP & 15.26 & $\mathrm{RI}, \mathrm{MS}$ & $\begin{array}{r}0.001 \\
\pm(0.002)\end{array}$ & $\begin{array}{r}0 \\
\pm(0.001)\end{array}$ & $\begin{array}{r}0.002 \\
\pm(0.002)\end{array}$ & $\begin{array}{r}0.006 \\
\pm(0.011)\end{array}$ \\
\hline L26 & $\begin{array}{l}\text { trans-Cadina- } \\
\text { 1,4-diene }\end{array}$ & TRP & 15.37 & $\begin{array}{l}\text { RI, MS, } \\
\text { REF }\end{array}$ & $\begin{array}{r}0.001 \\
\pm(0.002)\end{array}$ & $0 \pm(0)$ & $\begin{array}{r}0.002 \\
\pm(0.003)\end{array}$ & $\begin{array}{r}0.003 \\
\pm(0.004)\end{array}$ \\
\hline L27 & $\alpha$-Calacorene & $\begin{array}{l}\text { TRP, } \\
\text { AR }\end{array}$ & 15.47 & $\mathrm{RI}, \mathrm{MS}$ & $\begin{array}{r}0 \\
\pm(0.001)\end{array}$ & $0 \pm(0)$ & $\begin{array}{r}0.001 \\
\pm(0.001)\end{array}$ & $\begin{array}{r}0.002 \\
\pm(0.002)\end{array}$ \\
\hline & TOTAL & & & & $\begin{array}{r}0.11 \\
\pm(0.21)\end{array}$ & $\begin{array}{r}0.04 \\
\pm(0.04)\end{array}$ & $\begin{array}{r}8.38 \\
\pm(9.07)\end{array}$ & $\begin{array}{r}0.53 \\
\pm(0.86)\end{array}$ \\
\hline
\end{tabular}


Appendix 1 - supplementary materials for chapter 3
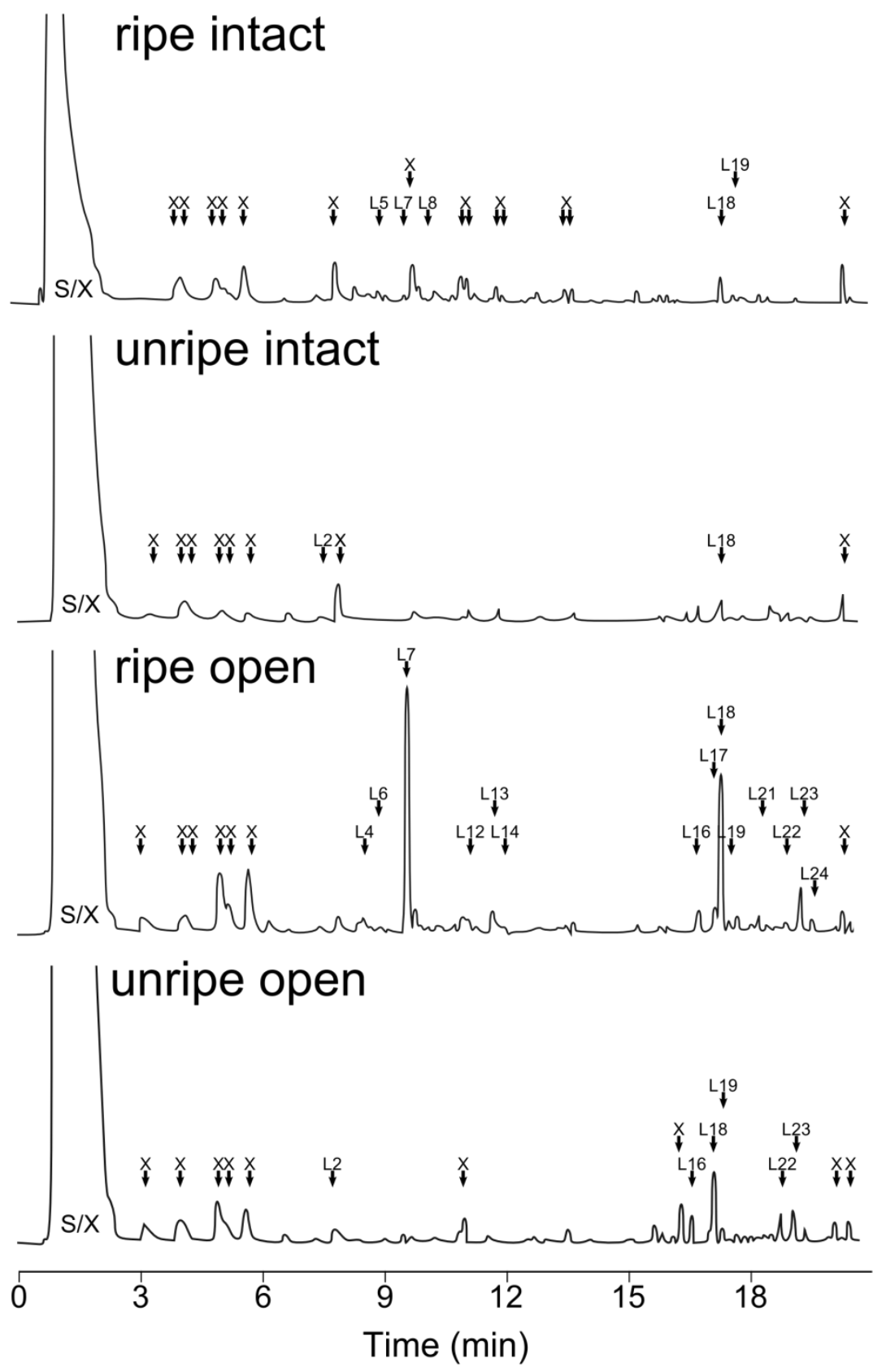

Fig. 8.2. Representative gas chromatograms of odor profiles of $\boldsymbol{L}$. cymosa. Compound names are shown in Tab. 8.2. X marks contaminants. SX marks a solvent \& unknown contaminant peaks present in all samples, including control. Presented samples were chosen according to their proximity to the group means (e.g. ripe intact). However, they may markedly deviate from the group mean with regards to some individual compounds and are thus for illustration only. 
Appendix 1 - supplementary materials for chapter 3

Tab. 8.3. Compounds identified from Psychotria cincta. Mean ( $\pm S D$ ) estimated absolute amounts (mg) of compounds in all Psychotria cincta samples in $\mathrm{mg}$ after $2.5 \mathrm{~h}$ sampling and retention indices (RI). Absolute amounts were calculated based on an external standard. Values are rounded and thus very small amounts are sometimes presented as 0 . Compounds presented are those present in at least 8 samples in a species (see Methods). ID method: methods used for identification of the respective compound. RI: retention index matching published values. MS: strong match with published mass spectra. REF: compound identification verified by running a synthetic compound or, in cases of enantiomers and close derivatives, a mixture of known compounds. In unknown compounds, ID method provides dominant MS ions. Compound classes: terpenoids (TRP), aromatic compounds (AR), aldehydes (ALD), alcohols (ALC), esters (EST), acids (AC), ketones (KET)

\begin{tabular}{|c|c|c|c|c|c|c|c|c|c|}
\hline Code & ID & Class & $\mathbf{R I}$ & \multicolumn{2}{|c|}{$\begin{array}{l}\text { ID } \\
\text { method }\end{array}$} & $\begin{array}{l}\text { Ripe } \\
\text { intact }\end{array}$ & $\begin{array}{l}\text { Unripe } \\
\text { intact }\end{array}$ & $\begin{array}{l}\text { Ripe } \\
\text { open }\end{array}$ & $\begin{array}{l}\text { Unripe } \\
\text { open }\end{array}$ \\
\hline P1 & $\begin{array}{l}\text { Unknown } \\
\text { C3- } \\
\text { benzene }\end{array}$ & AR & 9.53 & \multicolumn{2}{|l|}{ MS } & $\begin{array}{r}0.009 \\
\pm(0.028)\end{array}$ & $\begin{array}{r}0.007 \\
\pm(0.018)\end{array}$ & $\begin{array}{r}0.04 \\
\pm(0.08)\end{array}$ & $0 \pm(0)$ \\
\hline P2 & $\begin{array}{l}\text { Unknown } \\
\text { C3- } \\
\text { benzene }\end{array}$ & AR & 9.63 & \multicolumn{2}{|l|}{ MS } & $\begin{array}{r}0.003 \\
\pm(0.006)\end{array}$ & $\begin{array}{r}0.006 \\
\pm(0.009)\end{array}$ & $\begin{array}{r}0.008 \\
\pm(0.017)\end{array}$ & $0 \pm(0)$ \\
\hline P3 & $\begin{array}{l}\text { 3- } \\
\text { Octanone }\end{array}$ & KET & 9.86 & \multicolumn{2}{|c|}{ MS, RI } & $0 \pm(0)$ & $0 \pm(0)$ & $\begin{array}{r}0.017 \\
\pm(0.034)\end{array}$ & $\begin{array}{r}0.021 \\
\pm(0.025)\end{array}$ \\
\hline P4 & Myrcene & TRP & 9.90 & $\begin{array}{l}\text { MS, } \\
\text { REF }\end{array}$ & $\mathrm{RI}$, & $\begin{array}{r}0.005 \\
\pm(0.016)\end{array}$ & $\begin{array}{r}0.004 \\
\pm(0.013)\end{array}$ & $\begin{array}{r}0.001 \\
\pm(0.004)\end{array}$ & $\begin{array}{r}0.018 \\
\pm(0.039)\end{array}$ \\
\hline P5 & Mesitylene & AR & 9.94 & \multicolumn{2}{|l|}{ MS, RI } & $\begin{array}{r}0.003 \\
\pm(0.006)\end{array}$ & $\begin{array}{r}0.008 \\
\pm(0.011)\end{array}$ & $\begin{array}{r}0.007 \\
\pm(0.013)\end{array}$ & $0 \pm(0)$ \\
\hline P6 & $p$-Cymene & $A R, T R P$ & 10.25 & $\begin{array}{l}\text { MS, } \\
\text { REF }\end{array}$ & $\mathrm{RI}$, & $\begin{array}{r}0.024 \\
\pm(0.075)\end{array}$ & $\begin{array}{r}0.009 \\
\pm(0.029)\end{array}$ & $0 \pm(0)$ & $\begin{array}{r}0.001 \\
\pm(0.003)\end{array}$ \\
\hline P7 & $\begin{array}{l}(E)- \\
\beta-\text { Ocimene }\end{array}$ & TRP & 10.47 & $\begin{array}{l}\text { MS, } \\
\text { REF }\end{array}$ & $\mathrm{RI}$, & $0 \pm(0)$ & $\begin{array}{r}0.006 \\
\pm(0.013)\end{array}$ & $0 \pm(0)$ & $\begin{array}{r}0.009 \\
\pm(0.014)\end{array}$ \\
\hline P8 & Linalool & TRP, ALC & 11.01 & $\begin{array}{l}\text { MS, } \\
\text { REF }\end{array}$ & $\mathrm{RI}$, & $0 \pm(0)$ & $0 \pm(0)$ & $\begin{array}{r}0.036 \\
\pm(0.045)\end{array}$ & $\begin{array}{r}0.243 \\
\pm(0.237)\end{array}$ \\
\hline P9 & Nonanal & ALD & 11.06 & $\begin{array}{l}\text { MS, } \\
\text { REF }\end{array}$ & $\mathrm{RI}$, & $\begin{array}{r}0.007 \\
\pm(0.009)\end{array}$ & $\begin{array}{r}0.009 \\
\pm(0.01)\end{array}$ & $\begin{array}{r}0.011 \\
\pm(0.014)\end{array}$ & $\begin{array}{r}0.003 \\
\pm(0.006)\end{array}$ \\
\hline P10 & $\alpha$-Copaene & TRP & 13.79 & $\begin{array}{l}\text { MS, } \\
\text { REF }\end{array}$ & $\mathrm{RI}$, & $0 \pm(0)$ & $\begin{array}{r}0.002 \\
\pm(0.003)\end{array}$ & $\begin{array}{r}0.001 \\
\pm(0.002)\end{array}$ & $\begin{array}{r}0.001 \\
\pm(0.002)\end{array}$ \\
\hline & TOTAL & & & & & $\begin{array}{r}0.05 \\
\pm(0.13)\end{array}$ & $\begin{array}{r}0.05 \\
\pm(0.07)\end{array}$ & $\begin{array}{r}0.12 \\
\pm(0.16)\end{array}$ & $\begin{array}{r}0.3 \\
\pm(0.3)\end{array}$ \\
\hline
\end{tabular}


Appendix 1 - supplementary materials for chapter 3
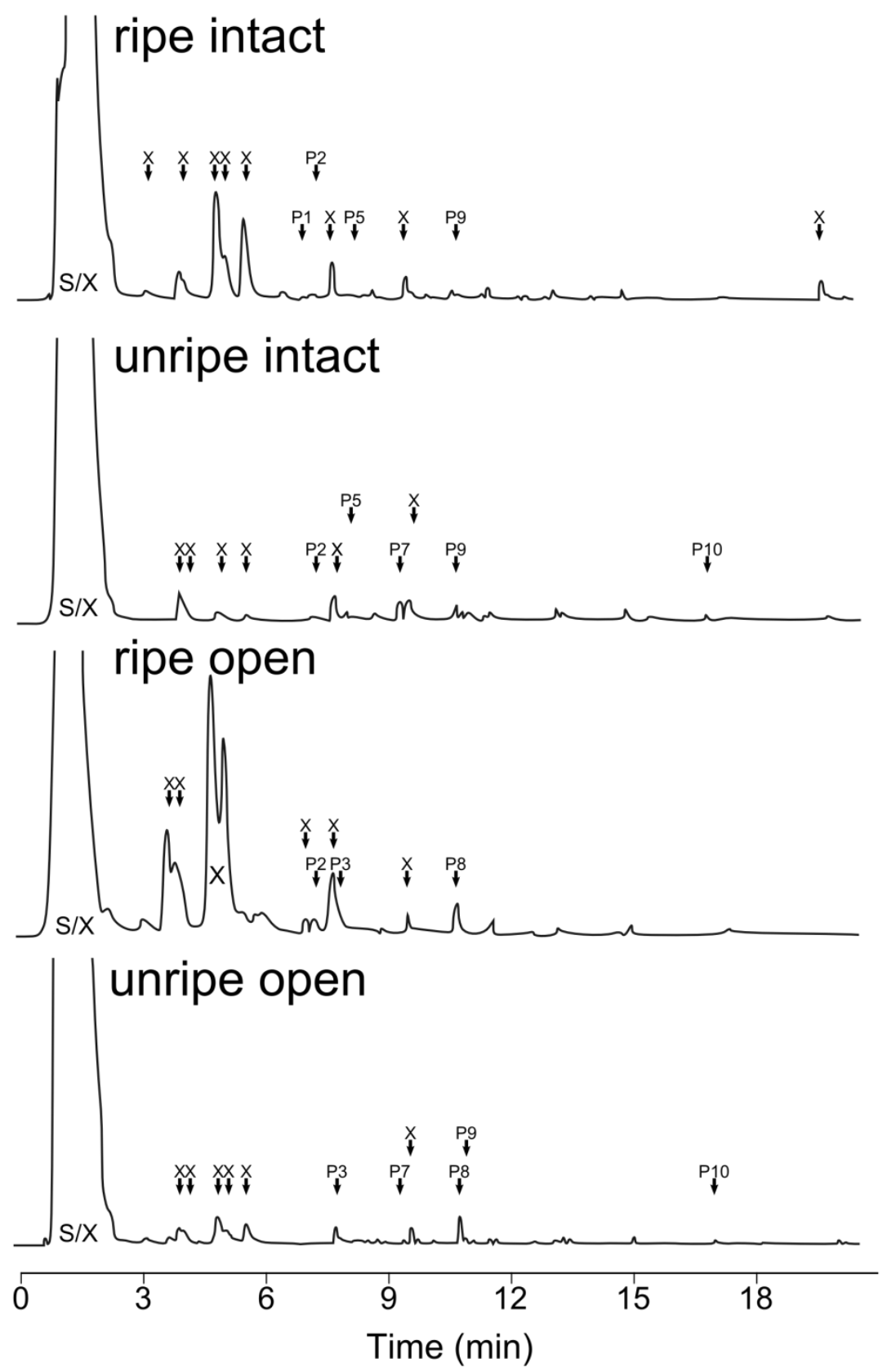

Fig. 8.3. Representative gas chromatograms of odor profiles of $\boldsymbol{P}$. cincta. Compound names are shown in Tab. 8.3. X marks contaminants. SX marks a solvent \& unknown contaminant peaks present in all samples, including control. Presented samples were chosen according to their proximity to the group means (e.g. ripe intact). However, they may markedly deviate from the group mean with regards to some individual compounds and are thus for illustration only. 
Appendix 1 - supplementary materials for chapter 3

Tab. 8.4. Compounds identified from Maieta guianensis. Mean ( $\pm S D$ ) estimated absolute amounts (mg) of compounds in all Maieta guianensis samples in $\mathrm{mg}$ after $2.5 \mathrm{~h}$ sampling and retention indices (RI). Absolute amounts were calculated based on an external standard. Values are rounded and thus very small amounts are sometimes presented as 0 . Compounds presented are those present in at least 8 samples in a species (see Methods). ID method: methods used for identification of the respective compound. RI: retention index matching published values. MS: strong match with published mass spectra. REF: compound identification verified by running a synthetic compound or, in cases of enantiomers and close derivatives, a mixture of known compounds. In unknown compounds, ID method provides dominant MS ions. Compound classes: terpenoids (TRP), aromatic compounds (AR), aldehydes (ALD), alcohols (ALC), esters (EST), acids (AC).

\begin{tabular}{|c|c|c|c|c|c|c|c|c|c|}
\hline Code & ID & Class & $\mathbf{R I}$ & $\begin{array}{l}\text { ID } \\
\text { meth }\end{array}$ & & $\begin{array}{l}\text { Ripe } \\
\text { intact }\end{array}$ & $\begin{array}{l}\text { Unripe } \\
\text { intact }\end{array}$ & $\begin{array}{l}\text { Ripe } \\
\text { open }\end{array}$ & $\begin{array}{l}\text { Unripe } \\
\text { open }\end{array}$ \\
\hline M1 & Cumene & $A R$ & 9.23 & $\begin{array}{l}\text { MS, } \\
\text { REF }\end{array}$ & $\mathrm{RI}$, & $\begin{array}{r}0.025 \\
\pm(0.047)\end{array}$ & $\begin{array}{r}0.017 \\
\pm(0.038)\end{array}$ & $\begin{array}{r}0.012 \\
\pm(0.039)\end{array}$ & $\begin{array}{r}0.023 \\
\pm(0.051)\end{array}$ \\
\hline M2 & $\begin{array}{l}\text { Unknown C3- } \\
\text { benzene }\end{array}$ & $A R$ & 9.53 & MS & & $\begin{array}{r}0.017 \\
\pm(0.031)\end{array}$ & $\begin{array}{r}0.009 \\
\pm(0.02)\end{array}$ & $\begin{array}{r}0.01 \\
\pm(0.033)\end{array}$ & $\begin{array}{r}0.015 \\
\pm(0.029)\end{array}$ \\
\hline M3 & $\begin{array}{l}\text { Unknown C3- } \\
\text { benzene }\end{array}$ & $A R$ & 9.62 & MS & & $\begin{array}{r}0.006 \\
\pm(0.012)\end{array}$ & $\begin{array}{r}0.003 \\
\pm(0.005)\end{array}$ & $\begin{array}{r}0.003 \\
\pm(0.011)\end{array}$ & $\begin{array}{r}0.007 \\
\pm(0.014)\end{array}$ \\
\hline M4 & Benzaldehyde & AR, ALD & 9.63 & $\begin{array}{l}\text { MS, } \\
\text { REF }\end{array}$ & $\mathrm{RI}$ & $\begin{array}{r}0.034 \\
\pm(0.096)\end{array}$ & $\begin{array}{r}0.031 \\
\pm(0.091)\end{array}$ & $\begin{array}{r}0.026 \\
\pm(0.071)\end{array}$ & $\begin{array}{r}0.045 \\
\pm(0.108)\end{array}$ \\
\hline M5 & $\begin{array}{l}\text { 1-Octen-3- } \\
\text { one }\end{array}$ & KET & 9.77 & $\mathrm{MS}, \mathrm{RI}$ & & $0 \pm(0)$ & $0 \pm(0)$ & $\begin{array}{r}0.023 \\
\pm(0.025)\end{array}$ & $\begin{array}{r}0.014 \\
\pm(0.028)\end{array}$ \\
\hline M6 & 1-Octen-3-ol & ALC & 9.82 & $\mathrm{MS}, \mathrm{RI}$ & & $0 \pm(0)$ & $0 \pm(0)$ & $\begin{array}{r}0.965 \\
\pm(0.822)\end{array}$ & $\begin{array}{r}0.356 \\
\pm(0.598)\end{array}$ \\
\hline M7 & 3-Octanone & KET & 9.86 & MS, RI & & $0 \pm(0)$ & $0 \pm(0)$ & $\begin{array}{r}0.216 \\
\pm(0.171)\end{array}$ & $\begin{array}{r}0.111 \\
\pm(0.18)\end{array}$ \\
\hline M8 & Mesitylene & $A R$ & 9.94 & MS, RI & & $\begin{array}{r}0.008 \\
\pm(0.013)\end{array}$ & $\begin{array}{r}0.008 \\
\pm(0.011)\end{array}$ & $\begin{array}{r}0.003 \\
\pm(0.011)\end{array}$ & $\begin{array}{r}0.007 \\
\pm(0.014)\end{array}$ \\
\hline M9 & 3-Octanol & ALC & 9.99 & MS, RI & & $0 \pm(0)$ & $0 \pm(0)$ & $\begin{array}{r}0.193 \\
\pm(0.244)\end{array}$ & $\begin{array}{r}0.026 \\
\pm(0.049)\end{array}$ \\
\hline M10 & p-Cymene & $A R$ & 10.25 & $\begin{array}{l}\text { MS, } \\
\text { REF }\end{array}$ & $\mathrm{RI}$, & $\begin{array}{r}0.006 \\
\pm(0.019)\end{array}$ & $\begin{array}{r}0.02 \\
\pm(0.06)\end{array}$ & $\begin{array}{r}0.022 \\
\pm(0.07)\end{array}$ & $\begin{array}{r}0.024 \\
\pm(0.06)\end{array}$ \\
\hline M11 & Nonanal & ALD & 11.06 & $\begin{array}{l}\text { MS, } \\
\text { REF }\end{array}$ & RI, & $\begin{array}{r}0.016 \\
\pm(0.011)\end{array}$ & $\begin{array}{r}0.002 \\
\pm(0.007)\end{array}$ & $\begin{array}{r}0.01 \\
\pm(0.009)\end{array}$ & $\begin{array}{r}0.017 \\
\pm(0.011)\end{array}$ \\
\hline & TOTAL & & & & & $\begin{array}{r}0.11 \\
\pm(0.2)\end{array}$ & $\begin{array}{r}0.09 \\
\pm(0.21)\end{array}$ & $\begin{array}{r}1.49 \\
\pm(1.04)\end{array}$ & $\begin{array}{r}0.64 \\
\pm(0.95)\end{array}$ \\
\hline
\end{tabular}


Appendix 1 - supplementary materials for chapter 3
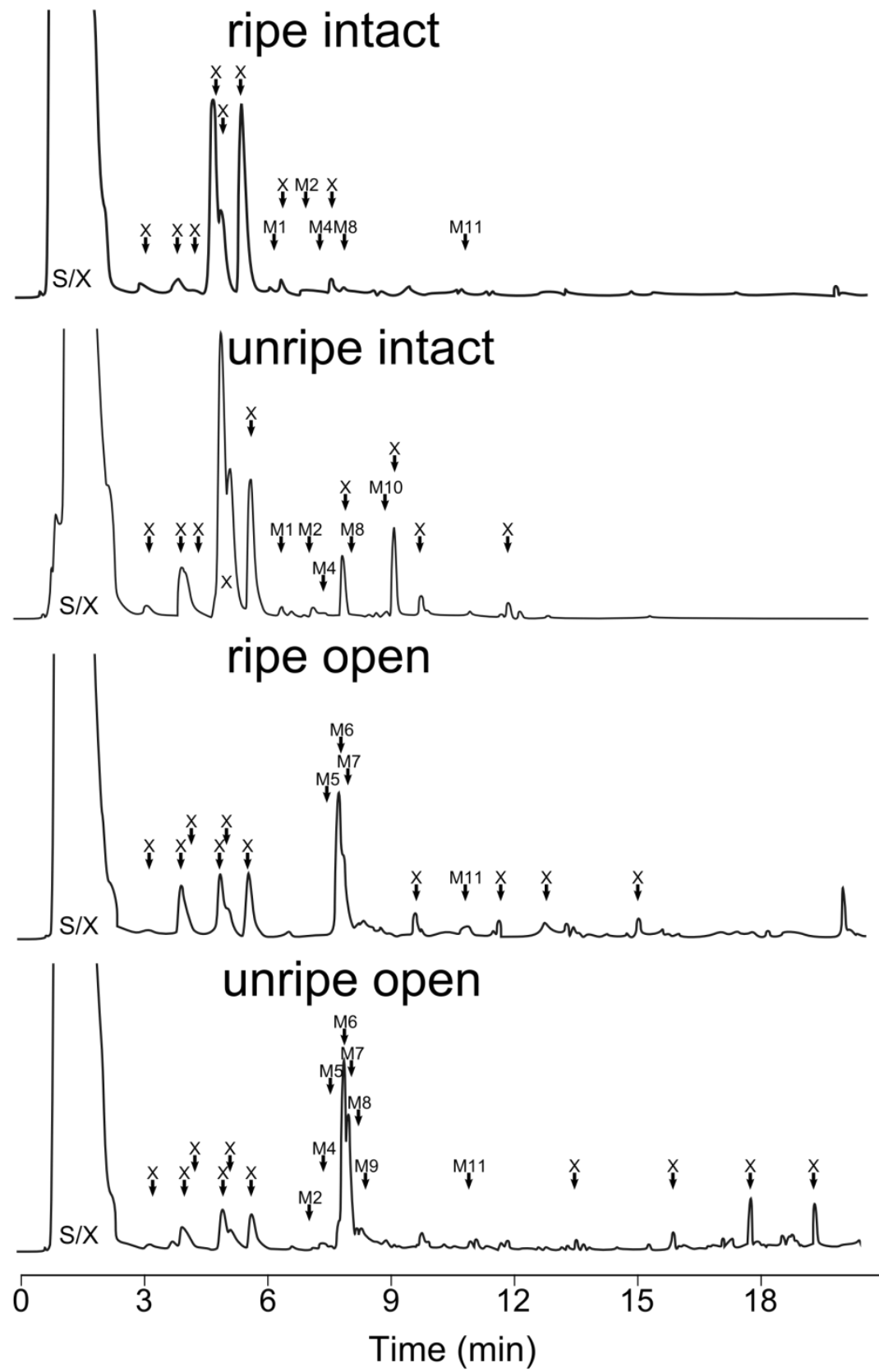

Fig. 8.4. Representative gas chromatograms of odor profiles of $\boldsymbol{M}$. guianensis. Compound names are shown in Tab. 8.4. X marks contaminants. SX marks a solvent \& unknown contaminant peaks present in all samples, including control. Presented samples were chosen according to their proximity to the group means (e.g. ripe intact). However, they may markedly deviate from the group mean with regards to some individual compounds and are thus for illustration only. 
Appendix 2 - supplementary materials for chapter 4

\section{APPENDIX 2 - SUPPLEMENTARY MATERIALS FOR CHAPTER 4}

Table 9.1: origin and purity of compounds used for all mixture preparations

\begin{tabular}{|c|c|c|c|}
\hline Common name & CAS & Purity & Origin \\
\hline Acetophenone & $98-86-2$ & $\geq 98 \%$ & $\begin{array}{l}\text { Sigma Aldrich, } \\
\text { Germany }\end{array}$ \\
\hline Benzaldehyde & $100-52-7$ & $\geq 98 \%$ & $\begin{array}{l}\text { Sigma Aldrich, } \\
\text { Germany }\end{array}$ \\
\hline (E-) Caryophyllene & $87-44-5$ & $80 \%$ & Dragon, China \\
\hline Cumene & $98-82-8$ & $98 \%$ & $\begin{array}{l}\text { Sigma Aldrich, } \\
\text { Germany }\end{array}$ \\
\hline Diethyl phthalate & $84-66-2$ & $\geq 99 \%$ & $\begin{array}{l}\text { Sigma Aldrich, } \\
\text { Germany }\end{array}$ \\
\hline D-Limonene & $5989-27-5$ & $98 \%$ & $\begin{array}{l}\text { Sigma Aldrich, } \\
\text { Germany }\end{array}$ \\
\hline Ethyl salicylate & $118-61-6$ & $99 \%$ & $\begin{array}{l}\text { Sigma Aldrich, } \\
\text { Germany }\end{array}$ \\
\hline$E$ - $\beta$-Ocimene & $3779-61-1$ & $90 \%+$ isomers & Dragon, China \\
\hline a-Humulene & $6753-98-6$ & $96 \%$ & $\begin{array}{l}\text { Sigma Aldrich, } \\
\text { Germany }\end{array}$ \\
\hline Linalool & $78-70-6$ & $97 \%$ & $\begin{array}{l}\text { Sigma Aldrich, } \\
\text { Germany }\end{array}$ \\
\hline Methyl salicytate & $119-36-8$ & $99 \%$ & $\begin{array}{l}\text { Sigma Aldrich, } \\
\text { Germany }\end{array}$ \\
\hline Myrcene & $123-35-3$ & $\geq 90 \%$ & $\begin{array}{l}\text { Sigma Aldrich, } \\
\text { Germany }\end{array}$ \\
\hline Nonanal & $124-19-6$ & $\geq 95 \%$ & $\begin{array}{l}\text { Sigma Aldrich, } \\
\text { Germany }\end{array}$ \\
\hline$p$-Cymenene & $1195-32-0$ & $\geq 98 \%$ & $\begin{array}{l}\text { Sigma Aldrich, } \\
\text { Germany }\end{array}$ \\
\hline p-Cymene & $99-87-6$ & $99 \%$ & $\begin{array}{l}\text { Sigma Aldrich, } \\
\text { Germany }\end{array}$ \\
\hline Sabinene & $3387-41-5$ & $75 \%$ & $\begin{array}{l}\text { Sigma Aldrich, } \\
\text { Germany }\end{array}$ \\
\hline Trans-2-nonenal & $18829-56-6$ & $97 \%$ & $\begin{array}{l}\text { Sigma Aldrich, } \\
\text { Germany }\end{array}$ \\
\hline a-Copaene & $138874-68-7$ & $70 \%$ & $\begin{array}{l}\text { ACC Corporation, CA, } \\
\text { US }\end{array}$ \\
\hline $\mathrm{y}$-Terpinene & $99-85-4$ & $97 \%$ & $\begin{array}{l}\text { Sigma Aldrich, } \\
\text { Germany }\end{array}$ \\
\hline
\end{tabular}


Appendix 2 - supplementary materials for chapter 4

Tab. 9.2: Recipe for odor mixtures mimicking the full odor of $C$. macrocarpa fruits. Amounts are in $\mu$ l to create a mixture of $2 \mathrm{ml}$. Odorant are from $100 \mathrm{mg} / \mathrm{ml}$ solutions.

\begin{tabular}{|c|c|c|}
\hline Odorant & Ripe intact $(\mu \mathrm{l})$ & Unripe intact $(\mu \mathrm{l})$ \\
\hline (E-) Caryophyllene & 94.92 & 28.57 \\
\hline a-Copaene & 94.92 & 228.57 \\
\hline$p$-Cymene & 0 & 1.14 \\
\hline Ethyl salicylate & 20.34 & 0 \\
\hline$\alpha$-Humulene & 6.78 & 11.43 \\
\hline D-Limonene & 0.20 & 5.71 \\
\hline Methyl salicytate & 20.34 & 0 \\
\hline Myrcene & 0.41 & 3.43 \\
\hline Nonanal & 0.68 & 0 \\
\hline Trans-2-nonenal & 6.78 & 0 \\
\hline$E$ - $\beta$-Ocimene & 2.71 & 0 \\
\hline Sabinene & 0 & 2.29 \\
\hline $\mathrm{Y}$-Terpinene & 0.14 & 1.14 \\
\hline Solvent (diethyl phthalate) & 1752 & 1718 \\
\hline
\end{tabular}

Tab. 9.3: Recipe for odor mixtures mimicking the full odor of $L$. cymosa fruits. Amounts are in $\mu$ to create a mixture of $2 \mathrm{ml}$. Odorant are from $100 \mathrm{mg} / \mathrm{ml}$ solutions.

\begin{tabular}{|c|c|c|c|c|}
\hline Odorant & Ripe intact ( $\mu \mathrm{l})$ & $\begin{array}{l}\text { Unripe intact } \\
(\mu \mathrm{l})\end{array}$ & Ripe open $(\mu \mathrm{l})$ & $\begin{array}{l}\text { Unripe open } \\
(\mu \mathrm{l})\end{array}$ \\
\hline Acetophenone & 2.51 & 4.44 & 0 & 1.95 \\
\hline Benzaldehyde & 2.82 & 2.54 & 9.76 & 6.35 \\
\hline a-Copaene & 5.64 & 3.17 & 14.63 & 39.07 \\
\hline Cumene & 0 & 1.59 & 0 & 0.32 \\
\hline$p$-Cymene & 0.09 & 0.32 & 0 & 0.24 \\
\hline$p$-Cymenene & 0 & 0 & 14.63 & 0.049 \\
\hline Myrcene & 0 & 0 & 0.098 & 0.12 \\
\hline$E$ - $\beta$-Ocimene & 2.67 & 0 & 536.58 & 0.32 \\
\hline$Z$ - $\beta$-Ocimene & 0 & 4.44 & 0 & 0 \\
\hline $\begin{array}{ll}\text { Solvent } & \text { (diethyl } \\
\text { phthalate) } & \end{array}$ & 1986 & 1983 & 1424 & 1951 \\
\hline
\end{tabular}




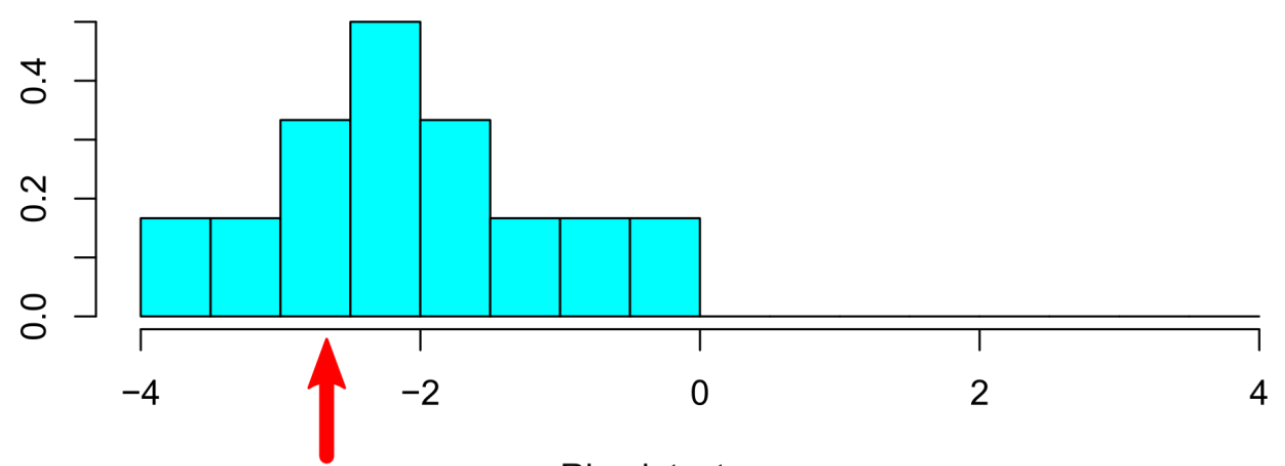

Ripe intact

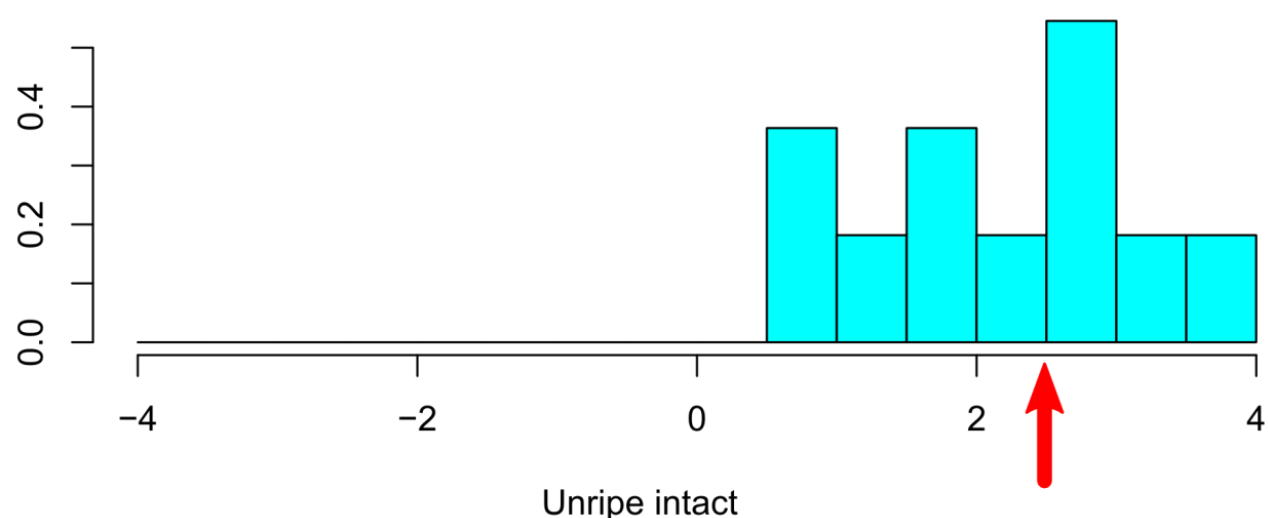

Fig. 9.1. Discriminant function analysis of odor of intact $C$. macrocarpa fruits. Data and analysis of natural fruit odor are identical to those in Nevo et al. Red arrows indicate the scores of the synthetic mixtures used in the bioassays in this study relative to their respective groups, and hence how representative they are. 
Appendix 2 - supplementary materials for chapter 4

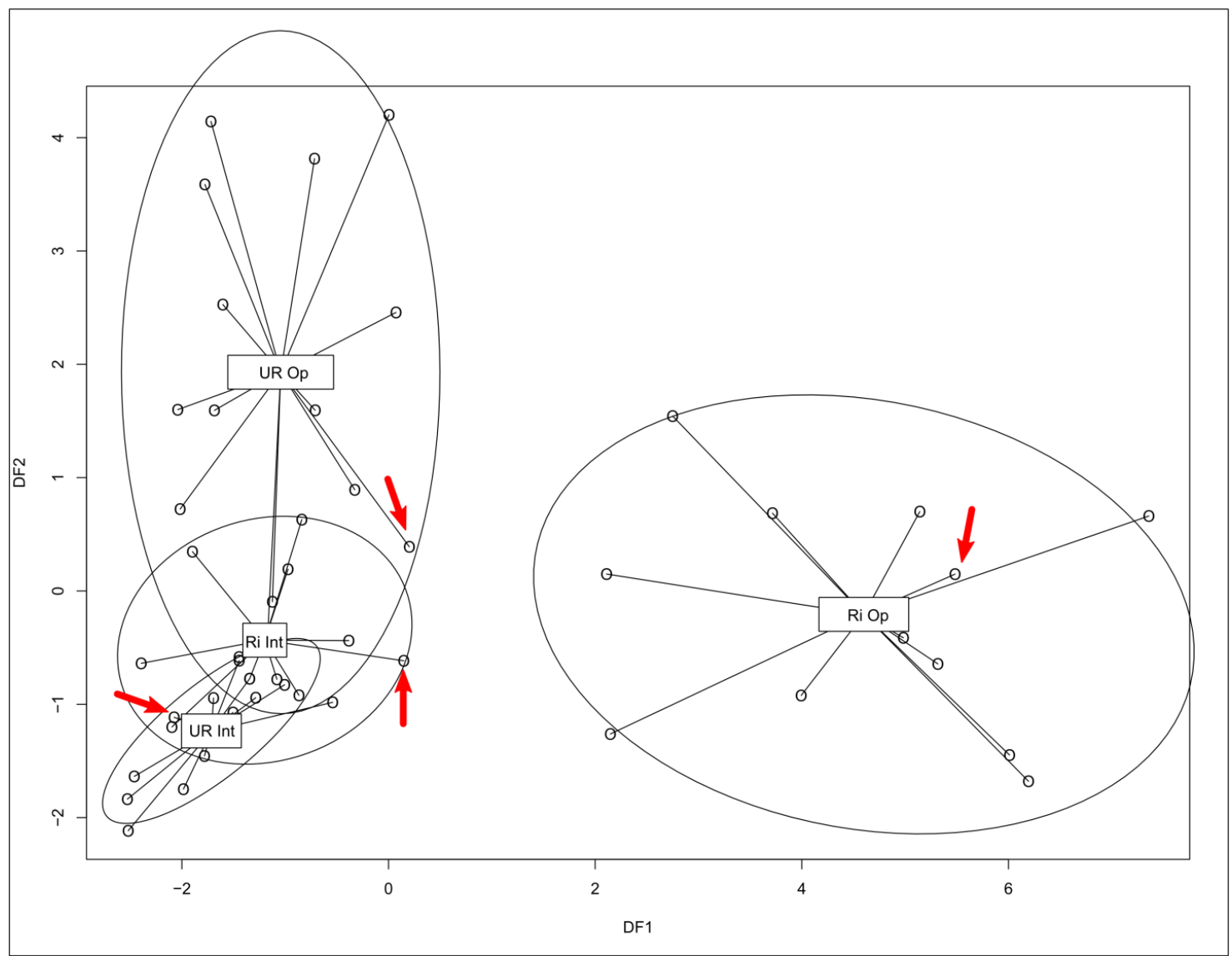

Fig. 9.2. Discriminant function analysis of odor of intact and open $L$. cymosa fruits. Data and analysis of natural fruit odor are identical to those in Nevo et al. Red arrows indicate the scores of the synthetic mixtures used in the bioassays in this study relative to their respective groups, and hence how representative they are. Ri Int: ripe intact; UR Int: unripe intact; Ri Op: ripe open; UR Op: unripe open. 
Curriculum vitae

\section{CURRICULUM VITAE}

Omer Nevo

Born 10.12.1984, Jerusalem

Dual Slovakian \& Israeli citizenship

\section{Contact details}

Private:

Gartenhalde 21

$89081 \mathrm{Ulm}$

Genomics

Germany

Professional:

University of Ulm

Institute of Evolutionary Ecology and Conservation

Helmholtzstr. 10-1

$89081 \mathrm{UIm}$

Germany

nevo84@gmail.com

+49(0)15756279508

omer.nevo@evolutionary-ecology.de

+49 (0)7315022662

\section{Education and academic work}

May 2015

Apr. 2011 - May 2015

Jun. 2011 - Aug. 2011

$2009-2010$

2004-2009

$1997-2003$
Post-doctoral researcher, Institute of Evolutionary Ecology and Conservation Genomics, University of UIm.

$\mathrm{PhD}$ student in Biology, Basic Biology GAUSS program, Division of Mathematics and Natural Sciences, Georg-August-Universität, Göttingen and German Primate Center (Deutsches Primatenzentrum), Göttingen, Germany.

Thesis title: "The Chemical Ecology of Primate Seed Dispersal". Grades: written thesis - Magna cum laude; Oral defense Summa cum laude; Final grade: Magna cum laude.

Research assistant, Behavioral Ecology \& Sociobiology Unit, German Primate Center (Deutsches Primatenzentrum), Göttingen, Germany.

MSc Human Evolution and Behaviour (first-class honours / distinction), Department of Anthropology, University College London, UK.

Master thesis title: "Olfaction in Primates Foraging: Interplays between Phylogeny and Ecology".

Sc, double major in Biology (final mark: 87) and Archaeology (95; Summa cum laude), Tel-Aviv University, Israel.

"Bagrut" graduate, Mathematics, English, Computer Science and Biology, Tzafit High School, Kfar-Menachem, Israel.

\section{Funding and awards}


2014

$2012-2013$

2012

2008
Minerva Fellowship Program - 1 y extension of funding for PhD studies

Minerva Fellowship Program - grant supporting PhD studies for $2 \mathrm{y}$.

Writing and designing the major part of a successful DFG research grant application submitted by PhD supervisors (HE 1870/19-1)

Award on behalf of the Department of Archaeology \& ANE Cultures for BA degree studies. Awarded for excellence in the 2007-2008 academic year.

\section{Publications}

Nevo O \& Heymann EW. 2015. Led by the nose: olfaction in primate feeding ecology. Evol Anthropol 24: 137-148.

Nevo O, Orts Garri R, Hernandez Salazar LT, Schulz S, Heymann EW, Ayasse M \& Laska M. 2015. Chemical recognition of fruit ripeness in spider monkeys (Ateles geoffroyi). Scientific Rep. 5: 14895.

Rathke E-M \& Nevo O. 2015. Short-term learning of olfactory discrimination tasks in cotton-top tamarins (Saguinus oedipus). Neotrop Primates 22: 12-18.

Nevo 0. In review (invited article). Olfactory communication. In International Encyclopedia of Primatology (Fuentes A, ed). Wiley Blackwell Publishers.

Nevo O, Heymann EW, Schulz S \& Ayasse M. In press. Fruit odor as a ripeness signal for seeddispersing primates? A case study on four Neotropical plant species. J Chem Ecol. DOI: 10.1007/s10886-016-0687-x.

Valenta K, Nevo O, Martel C, Miller CN \& Chapman C. In review (invited review). Plant attractants: integrating insights from seed dispersal and pollination ecology. Evol Ecol.

\section{Past \& ongoing collaborations}

Eckhard W. Heymann, German Primate Center

Manfred Ayasse, University of Ulm

Stefan Schulz, Technical University of Braunschweig

Matthias Laska, Linköping University

Laura Teresa Hernandez Salazar, University of Veracruzana

Amanda Melin, Washington University

Kim Valenta, McGill University

Marc de Meyer, Royal Museum for Central Africa, Tervuren

Peter Dürre, University of Ulm

\section{Presentations}


Oral presentation, symposium on odor communication between insects and plants, Konstanz, Oct 2015

Oral presentation, Society for Tropical Ecology congress, Zürich, Apr 2015

Guest speaker, Institute of Evolutionary Ecology and Conservation Genomics, University of Ulm, Germany.13 NOV 2014 "Is fruit aroma an adaptation to primate seed dispersal?"

Poster presentation, ISCE CSiV meeting, Urbana, IL, US, Jul 2014

Poster presentation, BioDevEvo conference, Dresden, Germany, Mar 2014

\section{Involvement in communication between scientists}

In process: co-organizing a session on primate sensory ecology in the 2016 joint meeting of the American Society of Primatologists and the International Primatological Society.

Co-organizing a session in seed dispersal in the annual meeting of the Society for Tropical Ecology meeting, Zürich, Apr 2015.

\section{Membership in scientific societies}

International Society of Chemical Ecology

Society of Tropical Ecology

\section{Teaching and supervision}

Supervision of 3 Diploma (equivalent to MA) students in a 3-week module on animal-plant interactions, University of UIm. 2015. Teaching scientific project design, field work methods, data collection, chemical sampling and analysis, data analysis and presentation.

Teaching field methods in chemical ecology, Peru, 2013. Field methods in sample collection.

Supervision of Diploma in biology, UNAP, Peru. 2013. Project deign, field methods, data collection and analysis.

Supervision of BSc in biology thesis, University of Göttingen. 2012. Project design, data collection and analysis, data presentation.

\section{Non-scientific work \& voluntary experience}

2007-2009

2002-2006

\section{Other skills}

Court verdicts filtering, coding and editing, Nevo Publishing Itd. (www.nevo.co.il).

Database programming and maintenance, Physicians for Human Rights Israel (www.phr.org.il/default.asp?PagelD=4), voluntary work. 
Research

Languages

Computers
Various methods of sampling and analysis in chemical ecology: dynamic and static headspace sampling in the field and the lab; GC / GC-MS analysis.

Zoological, ecological and botanical field data collection, EBQB, Peru (Sep - Oct 2011, May - Oct 2013, Feb - Jul 2013)

Behavioral study of captive primates, Heidelberg Zoo (Apr.- Jul. 2010).

Some experience with archaeological methodology.

Hebrew (first language), English (academic level), German (intermediate) and basic Spanish.

Intermediate level in Visual Basic programming and SQL (self educated). Experience in database development and management (MS Access).

Intermediate level in programming and statistical analysis in $\mathrm{R}$ environment.

Basic level of programing in $\mathrm{C}++$.

Experience in graphic environments (Adobe Photoshop and Illustrator). 
Declaration

\section{DECLARATION}

I hereby declare that I have written this thesis ("The Chemical Ecology of Primate Seed Dispersal") independently and with no aides other than the sources quoted.

Omer Nevo, Ulm, Mar 2016
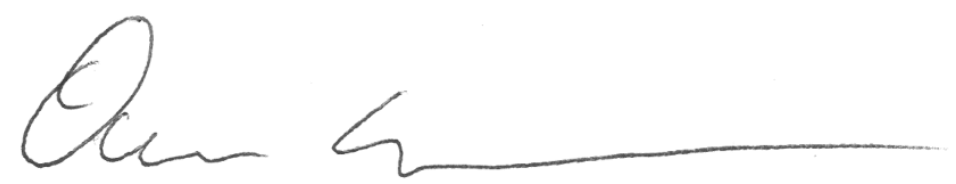